\title{
Global well-posedness of partially periodic KP-I equation in the energy space and application
}

\author{
Tristan Robert \\ Université de Cergy-Pontoise \\ Laboratoire AGM \\ 2 av. Adolphe Chauvin, 95302 Cergy-Pontoise Cedex, France \\ tristan.robert@u-cergy.fr
}

\begin{abstract}
In this article, we address the Cauchy problem for the KP-I equation

$$
\partial_{t} u+\partial_{x}^{3} u-\partial_{x}^{-1} \partial_{y}^{2} u+u \partial_{x} u=0
$$

for functions periodic in $y$. We prove global well-posedness of this problem for any data in the energy space $\mathbf{E}=\left\{u \in L^{2}, \partial_{x} u \in L^{2}, \partial_{x}^{-1} \partial_{y} u \in L^{2}\right\}$. We then prove that the $\mathrm{KdV}$ line soliton, seen as a special solution of KP-I equation, is orbitally stable under this flow, as long as its speed is small enough.
\end{abstract}

Keywords : Kadomtsev-Petviashvili equation, global well-posedness, orbital stability, KdV line soliton.

\section{Introduction}

\subsection{Motivations}

The Kadomtsev-Petviashvili equations

$$
\partial_{t} u+\partial_{x}^{3} u+\epsilon \partial_{x}^{-1} \partial_{y}^{2} u+u \partial_{x} u=0
$$

were first introduced in [12] as two-dimensional generalizations of the Korteweig-de Vries equation

$$
\partial_{t} u+\partial_{x}^{3} u+u \partial_{x} u=0
$$

They model long, weakly nonlinear waves propagating essentially along the $x$ direction with a small dependence in the $y$ variable. The coefficient $\epsilon \in\{-1 ; 1\}$ takes into account the surface tension. When this latter is strong $(\epsilon=-1),(1.1)$ is then called KP-I equation, whereas KP-II equation refers to a small surface tension $(\epsilon=+1)$.

The KdV equation (1.2) admits a particular family of traveling waves solutions, the so-called solitons $Q_{c}(x-c t)$ with speed $c>0$ :

$$
Q_{c}(x):=3 c \cdot \cosh \left(\frac{\sqrt{c}}{2} x\right)^{-2}
$$


From the work of Benjamin [2], we know that these solutions are orbitally stable in $H^{1}(\mathbb{R})$ under the flow generated by the $\mathrm{KdV}$ equation (1.2), meaning that every solution of (1.2) with initial data close to $Q_{c}$ in $H^{1}(\mathbb{R})$ remains close in $H^{1}(\mathbb{R})$ to the $Q_{c}$-orbit (under the action of translations) at any time $t>0$.

Looking at (1.1), we see that every solution of the KdV equation (1.2) is a solution of the KP equations (1.1), seen as a function independent of $y$. It is then a natural question to ask whether $Q_{c}$ is orbitally stable or unstable under the flow generated by (1.1). In order to do so, we first need a global well-posedness theory for (1.1) in a space containing $Q_{c}$. In particular, this rules out any well-posedness result in an anisotropic Sobolev space $H^{s_{1}, s_{2}}\left(\mathbb{R}^{2}\right)$. A more suited space to look for is the energy space for functions periodic in $y$ :

$$
\mathbf{E}(\mathbb{R} \times \mathbb{T}):=\left\{u_{0}(x, y) \in L^{2}(\mathbb{R} \times \mathbb{T}), \quad \partial_{x} u_{0} \in L^{2}(\mathbb{R} \times \mathbb{T}), \quad \partial_{x}^{-1} \partial_{y} u_{0} \in L^{2}(\mathbb{R} \times \mathbb{T})\right\}
$$

where $\mathbb{T}=\mathbb{R} / 2 \pi \mathbb{Z}$. Indeed, due to the Hamiltonian structure of (1.1), the mass

$$
\mathcal{M}(u)(t):=\int_{\mathbb{R} \times \mathbb{T}} u^{2}(t, x, y) \mathrm{d} x \mathrm{~d} y
$$

and the energy

$$
\mathcal{E}(u)(t):=\int_{\mathbb{R} \times \mathbb{T}}\left\{\left(\partial_{x} u\right)^{2}(t, x, y)+\left(\partial_{x}^{-1} \partial_{y} u\right)^{2}(t, x, y)-\frac{1}{3} u^{3}(t, x, y)\right\} \mathrm{d} x \mathrm{~d} y
$$

are (at least formally) conserved by the flow, i.e. $\mathcal{M}(u)(t)=\mathcal{M}(u)(0)$ and $\mathcal{E}(u)(t)=\mathcal{E}(u)(0)$, for any time $t$ and any solution $u$ of the KP-I equation defined on $[0, t]$. The conservation of the energy allows one to extend local solutions in $\mathcal{C}([-T, T], \mathbf{E})$ into solutions globally defined. In this article, we thus focus on the following Cauchy problem for the KP-I equation set on $\mathbb{R} \times \mathbb{T}$ :

$$
\left\{\begin{array}{l}
\partial_{t} u+\partial_{x}^{3} u-\partial_{x}^{-1} \partial_{y}^{2} u+u \partial_{x} u=0, \quad(t, x, y) \in \mathbb{R}^{2} \times \mathbb{T} \\
u(t=0)=u_{0} \in \mathbf{E}(\mathbb{R} \times \mathbb{T})
\end{array}\right.
$$

\subsection{Well-posedness results}

The KP equations (1.1) have been extensively studied in the past few decades. Using a standard energy method, Iório and Nunes [10] proved existence and uniqueness of zero mean value solutions in $H^{s}, s>2$, for both KP equations on $\mathbb{R}^{2}$ and $\mathbb{T}^{2}$. From the point of view of well-posedness, the KP-II equation is much better understood. Indeed, since the pioneering work of Bourgain [3], we know that the KP-II equation is globally well-posed on both $L^{2}\left(\mathbb{R}^{2}\right)$ and $L^{2}\left(\mathbb{T}^{2}\right)$. On $\mathbb{R}^{2}$, Takaoka and Tzvetkov [23] and Isaza and Mejia [11] pushed the low regularity local well-posedness theory down to the anistropic Sobolev space $H^{s_{1}, s_{2}}\left(\mathbb{R}^{2}\right)$ with $s_{1}>-1 / 3, s_{2} \geqslant 0$. Later, Hadac [6] and then Hadac, Herr and Koch [7] reached the threshold $s_{1} \geqslant-1 / 2, s_{2} \geqslant 0$ which is the scaling critical regularity for the KP-II equation. As for the initial value problem on $\mathbb{R} \times \mathbb{T}$, in order to study the stability of the KdV soliton under the flow of the KP-II equation, Molinet, Saut and Tzvetkov [19] proved global well-posedness on $L^{2}(\mathbb{R} \times \mathbb{T})$.

The situation is radically different regarding the Cauchy theory for the KP-I equation. From the work of Molinet, Saut and Tzvetkov [18], we know that this equation badly behaves with respect to pertubation methods. In particular, it is not possible to get well-posedness of (1.6) using the standard Fourier restriction norm method of Bourgain, nor any method using a fixed point argument on the Duhamel formula associated with (1.6) since Koch and 
Tzvetkov [15] proved that on $\mathbb{R}^{2}$, the flow map even fails to be uniformly continuous on bounded sets of $\mathcal{C}([-T, T], \mathbf{E})$. It is thus expected to have the same ill-posedness result on $\mathbb{R} \times \mathbb{T}$. Using the refined energy method introduced in [16], Kenig [13], and then Ionescu and Kenig [8] proved global well-posedness in the "second energy space"

$$
Z^{2}=:\left\{u \in L^{2}, \partial_{x}^{2} u \in L^{2}, \partial_{x}^{-2} \partial_{y}^{2} u \in L^{2}\right\}
$$

for functions on $\mathbb{R}^{2}$, and both $\mathbb{R} \times \mathbb{T}$ and $\mathbb{T}^{2}$, respectively. Lately, Ionescu, Kenig and Tataru [9] introduced the so-called short time Fourier restriction norm method and were able to prove global well-posedness of the KP-I equation in the energy space $\mathbf{E}\left(\mathbb{R}^{2}\right)$. Zhang [25] adapted this method in the periodic setting and got local well-posedness in the Besov space $\mathbf{B}_{2,1}^{1}\left(\mathbb{T}^{2}\right)$, which is almost the energy space but still strictly embedded in it. Overcoming the logarithmic divergence that appears in [25] to reach the energy space $\mathbf{E}\left(\mathbb{T}^{2}\right)$ is still an important open problem. In our case, we prove the following theorem, which answers the global well-posedness issue in the partially periodic setting :

\section{Theorem 1.1}

(a) Global well-posedness for smooth data

Take $u_{0} \in \mathbf{E}^{\infty}(\mathbb{R} \times \mathbb{T})$. Then, (1.6) admits a unique global solution

$$
u=\Phi^{\infty}\left(u_{0}\right) \in \mathcal{C}\left(\mathbb{R}, \mathbf{E}^{\infty}(\mathbb{R} \times \mathbb{T})\right)
$$

which defines a flow map

$$
\Phi^{\infty}: \mathbf{E}^{\infty}(\mathbb{R} \times \mathbb{T}) \rightarrow \mathcal{C}\left(\mathbb{R}, \mathbf{E}^{\infty}(\mathbb{R} \times \mathbb{T})\right)
$$

In addition, for any $T>0$ and $\alpha \in \mathbb{N}^{*}$,

$$
\left\|\Phi^{\infty}\left(u_{0}\right)\right\|_{L_{T}^{\infty} \mathbf{E}^{\alpha}} \leqslant C\left(T, \alpha,\left\|u_{0}\right\|_{\mathbf{E}^{\alpha}}\right)
$$

(b) Global well-posedness in the energy space

For any $u_{0} \in \mathbf{E}(\mathbb{R} \times \mathbb{T})$ and $T>0$, there exists a unique solution $u$ to (1.6) in the class

$$
\mathcal{C}([-T ; T], \mathbf{E}) \cap \mathbf{F}(T) \cap \mathbf{B}(T)
$$

Moreover, the corresponding global flow

$$
\Phi^{1}: \mathbf{E} \rightarrow \mathcal{C}(\mathbb{R}, \mathbf{E})
$$

is continuous and leaves $\mathcal{M}$ and $\mathcal{E}$ invariants.

The function spaces $\mathbf{E}_{\lambda}^{\alpha}, \mathbf{E}_{\lambda}^{\infty}, \mathbf{F}$ and $\mathbf{B}$ are defined in section 3 below.

\subsection{Stability results}

As far as stability issues are concerned, Mizumachi and Tzvetkov [17] proved that the KdV line soliton is stable under the flow generated by the KP-II equation on $L^{2}(\mathbb{R} \times \mathbb{T})$ for any speed $c>0$. Regarding the KP-I equation, Rousset and Tzvetkov [21] proved that $Q_{c}$ is orbitally unstable in $\mathbf{E}^{1}(\mathbb{R} \times \mathbb{T})$ under the KP-I flow constructed on $Z^{2}(\mathbb{R} \times \mathbb{T})$ in [8], whenever $c>c^{*}=4 / \sqrt{3}$, and that it is orbitally stable if $c<c^{*}$. Thus, as a byproduct of [21] and of 
our theorem 1.1, we can extend the range of admissible perturbations in [21, Theorem 1.4] to get

\section{Corollary 1.2}

Assume $c<4 / \sqrt{3}$, then $Q_{c}$ is orbitally stable in $\mathbf{E}$.

More precisely, for every $\varepsilon>0$, there exists $\delta>0$ such that for every $u_{0} \in \mathbf{E}(\mathbb{R} \times \mathbb{T})$ satisfying

$$
\left\|u_{0}-Q_{c}\right\|_{\mathbf{E}(\mathbb{R} \times \mathbb{T})}<\delta
$$

we have

$$
\sup _{t \in \mathbb{R}} \inf _{a \in \mathbb{R}}\left\|\Phi^{1}\left(u_{0}\right)(t, x-a, y)-Q_{c}(x-c t)\right\|_{\mathbf{E}(\mathbb{R} \times \mathbb{T})}<\varepsilon
$$

The proof of corollary 1.2 is a straightforward adaptation of the argument in [21]. Indeed, the proof of [21, Theorem 1.4] only uses the extra conditions $\partial_{x}^{2} u \in L^{2}, \partial_{x}^{-2} \partial_{y}^{2} u \in L^{2}$ to have the global solutions from [8]. For the sake of completeness, we present the outlines of the proof in section 9 .

\subsection{Strategy of the proof}

Let us now briefly discuss the main ingredients in the proof of theorem 1.1.

As pointed out above, it is irrelevant to look for functions spaces $\mathbf{F}(T) \hookrightarrow \mathcal{C}([-T, T], \mathbf{E})$ and $\mathbf{N}(T)$ such that any solution to (1.6) satisfies

1. a linear estimate

$$
\|u\|_{\mathbf{F}(T)} \lesssim\left\|u_{0}\right\|_{\mathbf{E}}+\|\left.\partial_{x}\left(u^{2}\right)\right|_{\mathbf{N}(T)}
$$

2. a bilinear estimate

$$
\left\|\left.\partial_{x}(u v)\right|_{\mathbf{N}(T)} \lesssim\right\| u\left\|_{\mathbf{F}(T)}\right\| v \|_{\mathbf{F}(T)}
$$

In order to construct solutions in $\mathbf{E}$, we will thus use the functions spaces $\mathbf{F}(T), \mathbf{N}(T)$ and $\mathbf{B}(T)$ introduced in [9]. Those spaces are built to combine the idea introduced in [16] of a priori estimates on short times (depending on the frequency) for frequency localized solutions, with the standard Bourgain spaces $X^{s, b}$ of [3]. Thus, we will replace (1.9)-(1.10) with

1. a linear estimate

$$
\|u\|_{\mathbf{F}(T)} \lesssim\|u\|_{\mathbf{B}(T)}+\left\|\partial_{x}\left(u^{2}\right)\right\|_{\mathbf{N}(T)}
$$

2. a bilinear estimate

$$
\left\|\left.\partial_{x}(u v)\right|_{\mathbf{N}(T)} \lesssim\right\| u\left\|_{\mathbf{F}(T)}\right\| v \|_{\mathbf{F}(T)}
$$

3. an energy estimate

$$
\|u\|_{\mathbf{B}(T)}^{2} \lesssim\left\|u_{0}\right\|_{\mathbf{E}}^{2}+\|u\|_{\mathbf{F}(T)}^{3}
$$

With (1.11)-(1.12)-(1.13) at hand, we will get the existence part of theorem 1.1 from a standard continuity argument.

To get uniqueness, we will prove the analogous of (1.11)-(1.12)-(1.13) for the difference equation, at the $L^{2}$ level :

$$
\begin{array}{r}
\|u-v\|_{\overline{\mathbf{F}}(T)} \lesssim\|u-v\|_{\overline{\mathbf{B}}(T)}+\left\|\partial_{x}\{(u-v)(u+v)\}\right\|_{\overline{\mathbf{N}}(T)} \\
\left.\left\|\partial_{x}\{(u-v)(u+v)\}\right\|\right|_{\overline{\mathbf{N}}(T)} \lesssim\|u-v\|_{\overline{\mathbf{F}}(T)}\|u+v\|_{\mathbf{F}(T)} \\
\|u-v\|_{\overline{\mathbf{B}}(T)}^{2} \lesssim\left\|u_{0}-v_{0}\right\|_{L^{2}}^{2}+\|u+v\|_{\mathbf{F}(T)}\|u-v\|_{\overline{\mathbf{F}}(T)}^{2}
\end{array}
$$


The main technical difficulties, compared to the case of $\mathbb{R}^{2}$, are the lack of a scale-invariant Strichartz estimate, and the impossibility to make the change of variables as in the proof of [9, Lemma 5.1 (a)] to estimate the volume of the resonant set. The first one is handled with frequency localized Strichartz estimates in the spirit of [3, 19]. For the second one, we follow Zhang [25, Lemma 3.1], but looking closely on the computations we are able to take advantage of the smallness of the intervals in which the frequency for the $x$ variable varies (note that this is not possible in [25] since this frequency lives in $\mathbb{Z}$ ) and to recover the same estimate as in [9] in this case. We also use a weighted Bourgain type space to deal with the logarithmic divergence in the energy estimate.

\subsection{Organization of the paper}

Sections 2 and 3 introduce general notations as well as functions spaces. We begin the proof of theorem 1.1 in section 4 by proving estimate (1.11). After establishing some general dyadic estimates in section 5, sections 6 and 7 deal with (1.12) and (1.13) respectively. The proof of theorem 1.1 is then completed in section 8. Finally, in the last section 9 we recall the arguments to obtain corollary 1.2 .

\section{Notations}

- We use the notations of [20] to deal with Fourier transform of periodic functions with a large period $2 \pi \lambda>0$. Let $\lambda \geqslant 1$ be fixed. We define $(\mathrm{d} q)_{\lambda}$ to be the renormalized counting measure on $\lambda^{-1} \mathbb{Z}$ :

$$
\int u(q)(\mathrm{d} q)_{\lambda}:=\frac{1}{\lambda} \sum_{q \in \lambda^{-1} \mathbb{Z}} u(q)
$$

In the sequel, all the Lebesgue norms in $q$ will be with respect to $(\mathrm{d} q)_{\lambda}$. Moreover, the space-time Lebesgue norms are defined as

$$
\|f\|_{L_{\xi, q}^{p} L_{\tau}^{r}}:=\left\{\int_{\mathbb{R} \times \lambda^{-1} \mathbb{Z}}\left(\int_{\mathbb{R}}|f|^{r} \mathrm{~d} \tau\right)^{p / r} \mathrm{~d} \xi(\mathrm{d} q)_{\lambda}\right\}^{1 / p}
$$

For a $2 \pi \lambda$-periodic function $f$, we define its Fourier transform as

$$
\widehat{f}(q):=\int_{0}^{2 \pi \lambda} \mathrm{e}^{-i q x} f(y) \mathrm{d} y, q \in \lambda^{-1} \mathbb{Z}
$$

and we have the inversion formula

$$
f(y)=\int \mathrm{e}^{i q y} \widehat{f}(q)(\mathrm{d} q)_{\lambda}
$$

We write $\mathbb{T}_{\lambda}:=\mathbb{R} / 2 \pi \lambda \mathbb{Z}$. Whenever $\lambda=1$ we drop the lambda.

- The Fourier transform of a function $u_{0}(x, y)$ on $\mathbb{R} \times \mathbb{T}_{\lambda}$ or $u(t, x, y)$ on $\mathbb{R}^{2} \times \mathbb{T}_{\lambda}$ is denoted $\widehat{u}$ or $\mathcal{F} u$ :

$$
\widehat{u_{0}}(\xi, q):=\int_{\mathbb{R} \times \mathbb{T}_{\lambda}} \mathrm{e}^{-i(\xi x+q y)} u_{0}(x, y) \mathrm{d} x \mathrm{~d} y, \quad(\xi, q) \in \mathbb{R} \times \lambda^{-1} \mathbb{Z}
$$


and

$$
\widehat{u}(\tau, \xi, q):=\int_{\mathbb{R}^{2} \times \mathbb{T}_{\lambda}} \mathrm{e}^{-i(\tau t+\xi x+q y)} u(t, x, y) \mathrm{d} t \mathrm{~d} x \mathrm{~d} y,(\tau, \xi, q) \in \mathbb{R}^{2} \times \lambda^{-1} \mathbb{Z}
$$

$\mathcal{F}_{t} u$ stands for the partial Fourier transform of $u(t, x, y)$ with respect to $t$, whereas $\mathcal{F}_{x y} u$ means the partial Fourier transform of $u$ with respect to space variables $x, y$, and similarly for $\mathcal{F}_{x}, \mathcal{F}_{y}$.

We always note $(\tau, \xi, q) \in \mathbb{R}^{2} \times \lambda^{-1} \mathbb{Z}$ the Fourier variables associated with $(t, x, y) \in$ $\mathbb{R}^{2} \times \mathbb{T}_{\lambda}$.

We note eventually $\zeta=(\xi, q) \in \mathbb{R} \times \lambda^{-1} \mathbb{Z}$.

- We denote $\star$ the convolution product for functions on $\mathbb{R}$ or $\lambda^{-1} \mathbb{Z}$ : to specify the variables,

$$
f\left(x^{\prime}\right) \star_{x} g\left(x^{\prime}\right) \text { means }(f \star g)(x)=\int_{\mathbb{R} \text { or } \lambda^{-1} \mathbb{Z}} f\left(x-x^{\prime}\right) g\left(x^{\prime}\right) \mathrm{d} x^{\prime}
$$

- We use the "bracket" notation $\langle\cdot\rangle$ for the weight in the definition of inhomogeneous Sobolev spaces, i.e

$$
\langle\xi\rangle^{s}:=\left(1+\xi^{2}\right)^{s / 2}
$$

- $U(t)$ is the unitary group defined by the linear evolution equation associated with (1.6)

$$
\forall u_{0} \in L^{2}\left(\mathbb{R} \times \mathbb{T}_{\lambda}\right), \quad \widehat{U(t) u_{0}}(\xi, q)=\mathrm{e}^{i t \omega(\xi, q)} \widehat{u_{0}}(\xi, q)
$$

where

$$
\omega(\xi, q):=\xi^{3}+q^{2} / \xi
$$

We also note

$$
\sigma(\tau, \xi, q):=\tau-\omega(\xi, q)=\tau-\xi^{3}-\frac{q^{2}}{\xi}
$$

the modulation associated with (1.6).

- For positive reals $a$ and $b, a \lesssim b$ means that there exists a positive constant $c>0$ (independent of the various parameters, including $\lambda$ ) such that $a \leqslant c \cdot b$. The notation $a \sim b$ stands for $a \lesssim b$ and $b \lesssim a$.

- We note $M \in \mathbb{R}_{+}^{*}$ (respectively $K \geqslant 1$ ) the dyadic frequency decomposition of $|\xi|$ (respectively of $\langle\sigma\rangle$ ), i.e $M \in 2^{\mathbb{Z}}$ and $K \in 2^{\mathbb{N}}$.

We define then

$$
D_{\lambda, M, K}:=\left\{(\tau, \xi, q) \in \mathbb{R}^{2} \times \lambda^{-1} \mathbb{Z}, \quad|\xi| \sim M,\langle\sigma(\tau, \xi, q)\rangle \sim K\right\}
$$

and

$$
D_{\lambda, M, \leqslant K}:=\left\{(\tau, \xi, q) \in \mathbb{R}^{2} \times \lambda^{-1} \mathbb{Z}, \quad|\xi| \sim M,\langle\sigma(\tau, \xi, q)\rangle \lesssim K\right\}=\bigcup_{K^{\prime} \leqslant K} D_{\lambda, M, K^{\prime}}
$$

We note also

$$
\mathfrak{I}_{M}:=\{M / 2 \leqslant|\xi| \leqslant 3 M / 2\}
$$

and

$$
\mathfrak{I}_{\leqslant M}:=\{|\xi| \leqslant 3 M / 2\}=\bigcup_{M^{\prime} \leqslant M} \mathfrak{I}_{M^{\prime}}
$$


- We use the notations $M_{1} \wedge M_{2}:=\min \left(M_{1}, M_{2}\right)$ and $M_{1} \vee M_{2}:=\max \left(M_{1}, M_{2}\right)$.

For $M_{1}, M_{2}, M_{3} \in \mathbb{R}_{+}^{*}, M_{\text {min }} \leqslant M_{\text {med }} \leqslant M_{\text {max }}$ denotes the increasing rearrangement of $M_{1}, M_{2}, M_{3}$, i.e

$$
\begin{aligned}
M_{\text {min }}:=M_{1} \wedge M_{2} \wedge M_{3}, \quad M_{\text {max }}= & M_{1} \vee M_{2} \vee M_{3} \\
& \text { and } M_{\text {med }}=M_{1}+M_{2}+M_{3}-M_{\text {max }}-M_{\text {min }}
\end{aligned}
$$

- We use two different Littlewood-Paley decompositions : the first one is homogeneous (on $2^{\mathbb{Z}}$ ) for $|\xi|$, the last one is inhomogeneous for $\langle\sigma\rangle \in 2^{\mathbb{N}}$.

Let $\chi \in \mathcal{C}_{0}^{\infty}(\mathbb{R})$ with $0 \leqslant \chi \leqslant 1$, supp $\chi \subset[-8 / 5 ; 8 / 5]$ and $\chi \equiv 1$ on $[-5 / 4 ; 5 / 4]$.

- For $M \in 2^{\mathbb{Z}}$, we then define $\eta_{M}(\xi):=\chi(\xi / M)-\chi(2 \xi / M)$, such that $\operatorname{supp} \eta_{M} \subset$ $\{5 / 8 M \leqslant|\xi| \leqslant 8 / 5 M\}$ and $\eta_{M} \equiv 1$ on $\{4 / 5 M \leqslant|\xi| \leqslant 5 / 4 M\}$. Thus $\xi \in$ $\operatorname{supp} \eta_{M} \Rightarrow \xi \in \mathfrak{I}_{M}$ and $|\xi| \sim M$.

- For $K \in 2^{\mathbb{N}}$, we also define $\rho_{1}(\sigma):=\chi(\sigma)$ and $\rho_{K}(\sigma):=\chi(\sigma / K)-\chi(2 \sigma / K)$, $K>1$, such that $\operatorname{supp} \rho_{K} \subset\{5 / 8 K \leqslant|\sigma| \leqslant 8 / 5 K\}$ and $\rho_{K} \equiv 1$ on $\{4 / 5 M \leqslant$ $|\sigma| \leqslant 5 / 4 K\}, K>1$. Thus $\sigma \in \operatorname{supp} \rho_{K} \Rightarrow\langle\sigma\rangle \sim K$.

- When needed, we may use other decompositions $\widetilde{\chi}, \widetilde{\eta}$ and $\widetilde{\rho}$ with the similar properties as $\chi, \eta, \rho$ and satisfying $\widetilde{\chi} \equiv 1$ on $\operatorname{supp} \chi, \widetilde{\eta} \equiv 1$ on supp $\eta$ and $\widetilde{\rho} \equiv 1$ on $\operatorname{supp} \rho$.

- Finally, for $\kappa \in \mathbb{R}_{+}^{*}$, we note $\chi_{\kappa}(x):=\chi(x / \kappa)$.

- We also define the Littlewood-Paley projectors associated with the previous decompositions :

$$
P_{M} u:=\mathcal{F}^{-1}\left(\eta_{M}(\xi) \widehat{u}\right) \text { and } P_{\leqslant M} u:=\sum_{M^{\prime} \leqslant M} P_{M} u=\mathcal{F}^{-1}\left(\chi_{M}(\xi) \widehat{u}\right)
$$

Moreover, we define

$$
P_{\text {Low }}:=P_{\leqslant 2^{-5}} \text { and } P_{\text {High }}:=1-P_{\text {Low }}
$$

- The energy space $\mathbf{E}_{\lambda}$ is defined as in (1.3) for any period $2 \pi \lambda$ :

$$
\mathbf{E}\left(\mathbb{R} \times \mathbb{\mathbb { T }}_{\lambda}\right):=\left\{u_{0} \in L^{2}\left(\mathbb{R} \times \mathbb{\mathbb { T }}_{\lambda}\right), \quad \partial_{x} u_{0} \in L^{2}\left(\mathbb{R} \times \mathbb{\mathbb { T }}_{\lambda}\right), \partial_{x}^{-1} \partial_{y} u_{0} \in L^{2}\left(\mathbb{R} \times \mathbb{T}_{\lambda}\right)\right\}
$$

It is endowed with the norm

$$
\left\|\left.u_{0}\right|_{\mathbf{E}_{\lambda}}:=\right\|\langle\xi\rangle \cdot p(\xi, q) \cdot \widehat{u_{0}} \|_{L^{2}}
$$

i.e $\mathbf{E}_{\lambda}$ is a weighted Sobolev space, with the weight defined as

$$
p(\xi, q):=\left\langle\langle\xi\rangle^{-1} q / \xi\right\rangle,(\xi, q) \in \mathbb{R} \times \lambda^{-1} \mathbb{Z}
$$

so that

$$
|\langle\xi\rangle \cdot p(\xi, q)|^{2}=1+\xi^{2}+\frac{q^{2}}{\xi^{2}}
$$

i.e

$$
\left\|u_{0}\right\|_{\mathbf{E}_{\lambda}}^{2}=\left\|u_{0}\right\|_{L^{2}}^{2}+\left\|\partial_{x} u_{0}\right\|_{L^{2}}^{2}+\left\|\partial_{x}^{-1} \partial_{y} u_{0}\right\|_{L^{2}}^{2}
$$


More generally, for $\alpha \in \mathbb{N}$, we define

$$
\mathbf{E}_{\lambda}^{\alpha}:=\left\{u_{0}(x, y) \in L^{2}\left(\mathbb{R} \times \mathbb{T}_{\lambda}\right), \quad\left\|u_{0}\right\|_{\mathbf{E}_{\lambda}^{\alpha}}:=\left\|\langle\xi\rangle^{\alpha} \cdot p(\xi, q) \cdot \widehat{u_{0}}\right\|_{L^{2}}<+\infty\right\}
$$

and

$$
\mathbf{E}_{\lambda}^{\infty}=\bigcap_{\alpha \in \mathbb{N}^{*}} \mathbf{E}_{\lambda}^{\alpha}
$$

- For a real $\xi$, we define

$$
[\xi]_{\lambda}:=\lambda^{-1}\lfloor\lambda \xi\rfloor \in \lambda^{-1} \mathbb{Z}
$$

- For a set $A \subset \mathbb{R}^{d}, \mathbb{1}_{A}$ is the characteristic function of $A$ and if $A$ is Lebesguemeasurable, $|A|$ means its measure. Similarly, if $A \subset \lambda^{-1} \mathbb{Z}$, its measure with respect to $(\mathrm{d} q)_{\lambda}$ will also be noted $|A|$. When $A \subset \mathbb{Z}$ is a finite set, its cardinal is denoted $\# A$.

- For $M>0$ and $s \in \mathbb{R}, \lesssim M^{s-}$ means $\leqslant C_{\varepsilon} M^{s-\varepsilon}$ for any choice of $\varepsilon>0$ small enough. We define similarly $M^{s+}$.

\section{Functions spaces}

\subsection{Definitions}

Let $M \in 2^{\mathbb{Z}}$.

First, the dyadic energy space is defined as

$$
E_{\lambda, M}:=\left\{u_{0} \in \mathbf{E}_{\lambda}^{0}, P_{M} u_{0}=u_{0}\right\}
$$

As in [9], for $M \in 2^{\mathbb{Z}}$ and $b_{1} \in[0 ; 1 / 2[$, the dyadic Bourgain type space is defined as

$$
\begin{aligned}
& X_{\lambda, M}^{b_{1}}:=\left\{f(\tau, \xi, q) \in L^{2}\left(\mathbb{R}^{2} \times \lambda^{-1} \mathbb{Z}\right), \operatorname{supp} f\right. \subset \mathbb{R} \times I_{M} \times \lambda^{-1} \mathbb{Z}, \\
&\left.\|f\|_{X_{\lambda, M}^{b_{1}}}:=\sum_{K \geqslant 1} K^{1 / 2} \beta_{M, K}^{b_{1}}\left\|\rho_{K}(\tau-\omega) f\right\|_{L^{2}}<+\infty\right\}
\end{aligned}
$$

where the extra weight $\beta_{M, K}$ is

$$
\beta_{M, K}:=1 \vee \frac{K}{(1 \vee M)^{3}}
$$

This weight, already used in $[3,19,5]$, allows to recover a bit of derivatives in the high modulation regime, thus preventing a logarithmic divergence in the energy estimate. Then, we use the $X_{\lambda, M}^{b_{1}}$ structure uniformly on time intervals of size $(1 \vee M)^{-1}$ :

$$
\begin{aligned}
& F_{\lambda, M}^{b_{1}}:=\left\{u(t, x, y) \in \mathcal{C}\left(\mathbb{R}, E_{\lambda, M}\right),\right. P_{M} u=u, \\
&\left.\|u\|_{F_{\lambda, M}^{b_{1}}}:=\sup _{t_{M} \in \mathbb{R}}\left\|p \cdot \mathcal{F}\left\{\chi_{(1 \vee M)^{-1}}\left(t-t_{M}\right) u\right\}\right\|_{X_{\lambda, M}^{b_{1}}}<+\infty\right\}
\end{aligned}
$$

and

$$
\begin{aligned}
& N_{\lambda, M}^{b_{1}}:=\left\{u(t, x, y) \in \mathcal{C}\left(\mathbb{R}, E_{\lambda, M}\right), P_{M} u=u,\right. \\
& \left.\quad\|u\|_{N_{\lambda, M}^{b_{1}}}:=\sup _{t_{M} \in \mathbb{R}}\left\|(\tau-\omega+i(1 \vee M))^{-1} p \cdot \mathcal{F}\left\{\chi_{(1 \vee M)^{-1}}\left(t-t_{M}\right) u\right\}\right\|_{X_{\lambda, M}^{b_{1}}}<+\infty\right\}
\end{aligned}
$$


For a function space $Y \hookrightarrow \mathcal{C}\left(\mathbb{R}, \mathbf{E}_{\lambda}^{\alpha}\right)$, we set

$$
Y(T):=\left\{u \in \mathcal{C}\left([-T, T], \mathbf{E}_{\lambda}^{\alpha}\right),\|u\|_{Y(T)}<+\infty\right\}
$$

endowed with

$$
\|u\|_{Y(T)}:=\inf \left\{\|\widetilde{u}\|_{Y}, \quad \widetilde{u} \in Y, \quad \widetilde{u} \equiv u \text { on }[-T, T]\right\}
$$

Finally, the main function spaces are defined as

$$
\begin{aligned}
\mathbf{F}_{\lambda}^{\alpha, b_{1}}(T):=\left\{u \in \mathcal{C}\left([-T, T], \mathbf{E}_{\lambda}^{\alpha}\right),\right. & \\
\|u\|_{\mathbf{F}_{\lambda}^{\alpha, b_{1}}(T)}:=\left(\sum_{M>0}(1 \vee M)^{2 \alpha}\left\|P_{M} u\right\|_{F_{\lambda, M}^{b_{1}}(T)}^{2}\right)^{1 / 2}<+\infty &
\end{aligned}
$$

and

$$
\begin{aligned}
& \mathbf{N}_{\lambda}^{\alpha, b_{1}}(T):=\left\{u \in \mathcal{C}\left([-T, T], \mathbf{E}_{\lambda}^{\alpha}\right),\right. \\
&\left.\|u\|_{\mathbf{N}_{\lambda}^{\alpha, b_{1}}(T)}:=\left(\sum_{M>0}(1 \vee M)^{2 \alpha}\left\|P_{M} u\right\|_{N_{\lambda, M}^{b_{1}}(T)}^{2}\right)^{1 / 2}<+\infty\right\}
\end{aligned}
$$

The last space is the energy space

$$
\begin{aligned}
\mathbf{B}_{\lambda}^{\alpha}(T):= & \left\{u \in \mathcal{C}\left([-T, T], \mathbf{E}_{\lambda}^{\alpha}\right),\right. \\
& \left.\|u\|_{\mathbf{B}_{\lambda}^{\alpha}(T)}:=\left(\left\|P_{\leqslant 1} u_{0}\right\|_{\mathbf{E}_{\lambda}^{\alpha}}^{2}+\sum_{M>1} \sup _{t_{M} \in[-T, T]}\left\|P_{M} u\left(t_{M}\right)\right\|_{\mathbf{E}_{\lambda}^{\alpha}}^{2}\right)^{1 / 2}<+\infty\right\}
\end{aligned}
$$

For $b_{1}=1 / 8$, we drop the exponent.

If moreover $\alpha=1$, we simply write $\mathbf{F}_{\lambda}(T), \mathbf{N}_{\lambda}(T)$ et $\mathbf{B}_{\lambda}(T)$.

We define similarly the spaces

$$
\overline{E_{\lambda, M}}, \overline{F_{\lambda, M}^{b_{1}}}, \overline{N_{\lambda, M}^{b_{1}}}
$$

which are the equivalents of $E_{\lambda, M}, F_{\lambda, M}^{b_{1}}, N_{\lambda, M}^{b_{1}}$ but on an $L^{2}$ level, i.e without the weight $p(\xi, q)$. In particular,

$$
\|u\|_{\mathbf{F}_{\lambda}(T)}^{2} \sim \sum_{M>0}(1 \vee M)^{2}\|u\| \frac{2}{F_{\lambda, M}^{b_{1}}(T)}+\left\|\partial_{x}^{-1} \partial_{y} u\right\| \frac{2}{F_{\lambda, M}^{b_{1}}(T)}
$$

For the difference equation, we will then use the $L^{2}$-type energy space

$$
\begin{aligned}
\overline{\mathbf{B}_{\lambda}}(T):=\left\{u \in \mathcal{C}\left([-T ; T], L^{2}\left(\mathbb{R} \times \mathbb{T}_{\lambda}\right)\right),\right. \\
\left.\|u\|_{\overline{\mathbf{B}_{\lambda}(T)}}^{2}:=\left\|P_{\leqslant 1} u_{0}\right\|_{L^{2}}+\sum_{M>1} \sup _{t_{M} \in[-T ; T]}\left\|P_{M} u\left(t_{M}\right)\right\|_{L^{2}}^{2}<+\infty\right\}
\end{aligned}
$$


and the spaces for the difference of solutions and for the nonlinearity are

$$
\begin{aligned}
\overline{\mathbf{F}}_{\lambda}^{b_{1}}(T):=\left\{u \in \mathcal{C}\left([-T ; T], L^{2}\left(\mathbb{R} \times \mathbb{T}_{\lambda}\right)\right),\right. & \\
\|u\|_{\mathbf{F}_{\lambda}}^{2}(T) & \left.:=\sum_{M>0}\left\|P_{M} u\right\| \frac{2}{F_{\lambda, M}^{b_{1}}(T)}<+\infty\right\}
\end{aligned}
$$

and

$$
\begin{aligned}
&{\overline{\mathbf{N}_{\lambda}}}^{b_{1}}(T):=\left\{u \in \mathcal{C}\left([-T ; T], L^{2}\left(\mathbb{R} \times \mathbb{T}_{\lambda}\right)\right),\right. \\
&\left.\|u\|_{{\overline{\mathbf{N}_{\lambda}}}^{b_{1}}(T)}^{2}:=\left.\sum_{M>0}\left\|P_{M} u\right\|\right|_{N_{\lambda, M}^{b_{1}}(T)} ^{2}<+\infty\right\}
\end{aligned}
$$

\subsection{Basic properties}

The following property of dyadic Bourgain type space is fundamental :

\section{Proposition 3.1}

Let $M \in 2^{\mathbb{Z}}, b_{1} \in\left[0 ; 1 / 2\left[, f_{M} \in X_{\lambda, M}^{b_{1}}\right.\right.$, and $\gamma \in L^{2}(\mathbb{R})$ satisfying

$$
|\widehat{\gamma(\tau)}| \lesssim\langle\tau\rangle^{-4}
$$

Then, for any $K_{0} \geqslant 1$ and $t_{0} \in \mathbb{R}$ :

$$
K_{0}^{1 / 2} \beta_{M, K_{0}}^{b_{1}}\left\|\chi_{K_{0}}(\tau-\omega) \mathcal{F}\left\{\gamma\left(K_{0}\left(t-t_{0}\right)\right) \mathcal{F}^{-1} f_{M}\right\}\right\|_{L^{2}} \lesssim \beta_{M, K_{0}}^{b_{1}}\left\|f_{M}\right\|_{X_{\lambda, M}^{0}}
$$

and

$$
\sum_{K \geqslant K_{0}} K^{1 / 2} \beta_{M, K}^{b_{1}}\left\|\rho_{K}(\tau-\omega) \mathcal{F}\left\{\gamma\left(K_{0}\left(t-t_{0}\right)\right) \mathcal{F}^{-1} f_{M}\right\}\right\|_{L^{2}} \lesssim\left\|f_{M}\right\|_{X_{\lambda, M}^{b_{1}}}
$$

and the implicit constants are independent of $K_{0}, t_{0}, M$ or $\lambda$.

We will have several uses of the following estimate

\section{Lemma 3.2}

For any $M \in 2^{\mathbb{Z}}$ and $f_{M} \in X_{\lambda, M}^{0}$, we have

$$
\left\|f_{M}\right\|_{L_{\xi, q}^{2} L_{\tau}^{1}} \lesssim\left\|f_{M}\right\|_{X_{\lambda, M}^{0}}
$$

\section{Proof :}

We decompose $f_{M}$ according to its modulations :

$$
\begin{aligned}
\left\|f_{M}\right\|_{L_{\xi, q}^{2} L_{\tau}^{1}} \leqslant \sum_{K \geqslant 1}\left\|\rho_{K}(\tau-\omega) f_{M}\right\|_{L_{\xi, q}^{2} L_{\tau}^{1}} & \\
& \lesssim \sum_{K \geqslant 1} K^{1 / 2}\left\|\widetilde{\rho_{K}}(\tau-\omega)\langle\tau-\omega\rangle^{-1 / 2} \cdot \rho_{K}(\tau-\omega) f_{M}\right\|_{L_{\xi, q}^{2} L_{\tau}^{1}}
\end{aligned}
$$


Next, using Cauchy-Schwarz inequality in the $\tau$ variable, we control the previous term with

$$
\sum_{K \geqslant 1} K^{1 / 2}\|\| \widetilde{\rho_{K}}(\tau-\omega)\langle\tau-\omega\rangle^{-1 / 2}\left\|\left.\right|_{L_{\tau}^{2}}\right\| \rho_{K}(\tau-\omega) f_{M}\left\|_{L_{\tau}^{2}}\right\|_{L_{\xi, q}^{2}}
$$

Now, since for any fixed $(\xi, q) \in \mathbb{R} \times \lambda^{-1} \mathbb{Z},\left\|\widetilde{\rho_{K}}(\tau-\omega)\langle\tau-\omega\rangle^{-1 / 2}\right\|_{L_{\tau}^{2}} \lesssim 1$, the sum above is finally estimated by

$$
\sum_{K \geqslant 1} K^{1 / 2}\left\|\rho_{K}(\tau-\omega) f_{M}\right\|_{L_{\xi, q, \tau}^{2}}=\left\|f_{M}\right\|_{X_{\lambda, M}^{0}}
$$

Now we prove the proposition.

\section{Proof :}

Let us begin by proving (3.10). Using that $\left\|\chi_{K_{0}}(\tau-\omega)\right\|_{L^{2}} \lesssim K_{0}^{1 / 2}$, we estimate the term on the left-hand side by

$$
\begin{aligned}
& K_{0}^{1 / 2} \beta_{M, K_{0}}^{b_{1}}\|\| \chi_{K_{0}}(\tau-\omega)\left\|_{L_{\tau}^{2}}\right\|\left(K_{0}^{-1} \mathrm{e}^{i \tau^{\prime} t_{0}} \widehat{\gamma}\left(K_{0}^{-1} \tau^{\prime}\right)\right) \star_{\tau} f_{M}\|\|_{L_{\tau}^{\infty}} \|_{L_{\xi, q}^{2}} \\
& \lesssim \beta_{M, K_{0}}^{b_{1}}\|\|\left(\mathrm{e}^{i \tau^{\prime} t_{0}} \widehat{\gamma}\left(K_{0}^{-1} \tau^{\prime}\right)\right) \star_{\tau} f_{M}\|\|_{L_{\tau}^{\infty}} \|_{L_{\xi, q}^{2}}
\end{aligned}
$$

(3.10) then follows from using Young's inequality $L^{\infty} \times L^{1} \rightarrow L^{\infty}$ and (3.12), since $\widehat{\gamma} \in L^{\infty}$ by the assumption (3.9).

Now we prove (3.11). We decompose $f_{M}$ according to its modulations and then distinguish two cases depending on the relation between $K$ and $K_{1}$ :

$$
\begin{aligned}
& \sum_{K \geqslant K_{0}} K^{1 / 2} \beta_{M, K}^{b_{1}}\left\|\rho_{K}(\tau-\omega) \mathcal{F}\left\{\gamma\left(K_{0}\left(t-t_{0}\right)\right) \mathcal{F}^{-1} f_{M}\right\}\right\|_{L^{2}} \\
& \leqslant \sum_{K \geqslant K_{0}} K^{1 / 2} \beta_{M, K}^{b_{1}} \sum_{K_{1} \geqslant 1}\left\|\rho_{K}(\tau-\omega)\left(\mathrm{e}^{i \tau^{\prime} t_{0}} \widehat{\gamma_{K_{0}^{-1}}}\right) \star_{\tau}\left(\rho_{K_{1}}(\tau-\omega) f_{M}\right)\right\|_{L^{2}} \\
& \quad=\sum_{K \geqslant K_{0}} \sum_{K_{1} \leqslant K / 10}()+\sum_{K \geqslant K_{0}} \sum_{K_{1} \gtrsim K}()=I+I I
\end{aligned}
$$

For the first term, we have $\left|\tau-\tau^{\prime}\right| \sim K$ since $|\tau-\omega| \sim K$ and $\left|\tau^{\prime}-\omega\right| \sim K_{1} \leqslant K / 10$, thus using Young inequality $L^{\infty} \times L^{1} \rightarrow L^{\infty}$, the estimate $\left\|\rho_{K}\right\|_{L^{2}} \lesssim K^{1 / 2}$ and then summing on $K \geqslant K_{0}$, we get the bound

$$
\begin{aligned}
& I \lesssim \sum_{K \geqslant K_{0}} K^{-1} \beta_{M, K}^{b_{1}} \sum_{K_{1} \leqslant K / 10}\left\|\rho_{K}(\tau-\omega)\left(\left|\tau^{\prime}\right|^{3 / 2} \widehat{\gamma_{K_{0}^{-1}}}\right) \star_{\tau}\left(\rho_{K_{1}}(\tau-\omega) f_{M}\right)\right\|_{L^{2}} \\
& \lesssim K_{0}^{-1 / 2} \sum_{K_{1} \leqslant K / 10}\left\|\left|\tau^{\prime}\right|^{3 / 2} \widehat{\gamma_{K_{0}^{-1}}}\left(\tau^{\prime}\right)\right\|_{L^{\infty}}\left\|\rho_{K_{1}}(\tau-\omega) f_{M}\right\|_{L_{\xi, q}^{2} L_{\tau}^{1}}
\end{aligned}
$$

This is enough for (3.11) after using (3.12) and

$$
\left\||\cdot|^{s} \widehat{\gamma_{K_{0}^{-1}}}\right\|_{L^{p}} \lesssim K_{0}^{s+1 / p-1}\left|\left\|\left.\cdot\right|^{s} \widehat{\gamma}\right\|_{L^{p}}\right.
$$


and the right-hand side is finite by the assumption on gamma (3.9).

Finally, $I I$ is simply controlled using Young $L^{1} \times L^{2} \rightarrow L^{2}$ and (3.13) :

$$
I I \lesssim \sum_{K_{1} \gtrsim K_{0}} K_{1}^{1 / 2} \beta_{M, K_{1}}^{b_{1}}\left\|\widehat{\gamma_{K_{0}^{-1}}}\right\|_{L^{1}}\left\|\rho_{K_{1}}(\tau-\omega) f_{M}\right\|_{L^{2}} \lesssim\left\|f_{M}\right\|_{X_{\lambda, M}^{b_{1}}}
$$

Remark 3.3. For the loss in (3.10) to be nontrivial, we need either $b_{1}=0$ or $K_{0} \lesssim(1 \vee M)^{3}$. In particular, in the multilinear estimates we cannot localize the term with the smallest frequency on time intervals of size $M_{\max }^{-1}$ when $b_{1}>0$.

The next proposition deals with general time multipliers as in [9] :

\section{Proposition 3.4}

Let $M>0, b_{1} \in\left[0 ; 1 / 2\left[, f_{M} \in F_{\lambda, M}^{b_{1}}\right.\right.$ (repectively $N_{\lambda, M}^{b_{1}}$ ) and $m_{M} \in \mathcal{C}^{4}(\mathbb{R})$ bounded along with its derivatives. Then

$$
\left\|m_{M}(t) f_{M}\right\|_{F_{\lambda, M}^{b_{1}}} \lesssim\left(\sum_{k=0}^{4}(1 \vee M)^{-k}\left\|m_{M}^{(k)}\right\|_{L^{\infty}}\right)\left\|f_{M}\right\|_{F_{\lambda, M}^{b_{1}}}
$$

and

$$
\left\|m_{M}(t) f_{M}\right\|_{N_{\lambda, M}^{b_{1}}} \lesssim\left(\sum_{k=0}^{4}(1 \vee M)^{-k}\left\|m_{M}^{(k)}\right\|_{L^{\infty}}\right)\left\|f_{M}\right\|_{N_{\lambda, M}^{b_{1}}}
$$

respectively, uniformly in $M>0$ and $\lambda \geqslant 1$.

\section{Proof :}

Using the definition of $F_{\lambda, M}^{b_{1}}$, we write

$$
\left\|m_{M} f_{M}\right\|_{F_{\lambda, M}^{b_{1}}}=\sup _{t_{M} \in \mathbb{R}} \sum_{K \geqslant 1} K^{1 / 2} \beta_{M, K}^{b_{1}}\left\|p \cdot \rho_{K}(\tau-\omega) \mathcal{F}\left\{\chi_{(1 \vee M)^{-1}}\left(t-t_{M}\right) m_{M}(t) f_{M}\right\}\right\|_{L^{2}}
$$

Next we estimate

$$
\left|\mathcal{F}\left\{\chi_{(1 \vee M)^{-1}}\left(t-t_{M}\right) m_{M}\right\}\right|(\tau) \leqslant\left\|\chi_{(1 \vee M)^{-1}}\left(t-t_{M}\right) m_{M}\right\|_{L^{1}} \lesssim(1 \vee M)^{-1}\left\|m_{M}\right\|_{L^{\infty}}
$$

and

$$
\begin{aligned}
\left|\mathcal{F}\left\{\chi_{(1 \vee M)^{-1}}\left(t-t_{M}\right) m_{M}\right\}\right|(\tau)=|\tau|^{-4} \mid & \mathcal{F} \frac{\mathrm{d}^{4}}{\mathrm{~d} t^{4}}\left\{\chi_{(1 \vee M)^{-1}}\left(t-t_{M}\right) m_{M}\right\} \mid \\
& \left.\lesssim|\tau|^{-4} \sum_{k=0}^{4}\left\|m_{M}^{(k)}\right\|_{L^{\infty}}(1 \vee M)^{3-k}\left\|\chi^{(4-k)}\right\|\right|_{L^{1}}
\end{aligned}
$$

Thus we obtain

$$
\begin{aligned}
\left|\mathcal{F}\left\{\chi_{(1 \vee M)^{-1}}\left(t-t_{M}\right) m_{M}\right\}\right| & (\tau) \\
& \lesssim\left(\sum_{k=0}^{4}(1 \vee M)^{-k}\left\|m_{M}^{(k)}\right\|_{L^{\infty}}\right)(1 \vee M)^{-1}\left\langle(1 \vee M)^{-1} \tau\right\rangle^{-4}
\end{aligned}
$$

Using (3.10) and (3.11) with $t_{0}=t_{M}, K_{0}=(1 \vee M)$ and $\gamma(t)=\mathcal{F}^{-1}\langle\tau\rangle^{-4}$ concludes the proof of (3.14). The proof of (3.15) follows similarly. 
The last proposition justifies the use of $\mathbf{F}_{\lambda}(T)$ as a resolution space :

\section{Proposition 3.5}

Let $\left.\left.\alpha \in \mathbb{N}^{*}, T \in\right] 0 ; 1\right], b_{1} \in\left[0 ; 1 / 2\left[\right.\right.$ and $u \in \mathbf{F}_{\lambda}^{\alpha, b_{1}}(T)$. Then

$$
\|u\|_{L_{T}^{\infty} \mathbf{E}_{\lambda}^{\alpha}} \lesssim\|u\|_{\mathbf{F}_{\lambda}^{\alpha, b_{1}}(T)}
$$

and

$$
\|u\|_{L_{T}^{\infty} L_{x y}^{2}} \lesssim\|u\|_{{\overline{\mathbf{F}_{\lambda}}}^{b_{1}}(T)}
$$

\section{Proof :}

The proof is the same as in [9, Lemma 3.1] : let $M \in 2^{\mathbb{Z}}, \widetilde{u_{M}}$ be an extension of $P_{M} u$ to $\mathbb{R}$ with $\left\|\widetilde{u_{M}}\right\|_{F_{\lambda, M}^{b_{1}}} \leqslant 2\left\|P_{M} u\right\|_{F_{\lambda, M}^{b_{1}}(T)}$ and $t_{M} \in[-T ; T]$, then it suffices to prove that

$$
\left\|\left.p \cdot \mathcal{F}_{x y} \widetilde{u_{M}}\left(t_{M}\right)\right|_{L_{\xi, q}^{2}} \lesssim\right\| p \cdot \mathcal{F}\left\{\chi_{(1 \vee M)^{-1}}\left(t-t_{M}\right) \widetilde{u_{M}}\right\} \|_{X_{\lambda, M}^{b_{1}}}
$$

Using the properties of $\chi$ and the inversion formula, we can write

$$
\widetilde{u_{M}}\left(t_{M}\right)=\left\{\chi_{(1 \vee M)^{-1}}\left(\cdot-t_{M}\right) \widetilde{u_{M}}\right\}\left(t_{M}\right)=\int_{\mathbb{R}} \mathcal{F}_{t}\left\{\chi_{(1 \vee M)^{-1}}\left(t-t_{M}\right) \widetilde{u_{M}}\right\}(\tau) \mathrm{e}^{i t_{M} \tau} \mathrm{d} \tau
$$

Thus, using (3.12), we get the final bound

$$
\begin{aligned}
&\left\|p \cdot \mathcal{F}_{x y} \widetilde{u_{M}}\left(t_{M}\right)\right\|_{L_{\xi, q}^{2}} \leqslant \| p \cdot \mathcal{F}\left\{\chi_{(1 \vee M)^{-1}}\left(t-t_{M}\right) \widetilde{u_{M}}\right\}||_{L_{\xi, q}^{2} L_{\tau}^{1}} \\
& \lesssim \|\left. p \cdot \mathcal{F}\left\{\chi_{(1 \vee M)^{-1}}\left(t-t_{M}\right) \widetilde{u_{M}}\right\}\right|_{X_{\lambda, M}^{b_{1}}}
\end{aligned}
$$

\section{Linear estimates}

This section deals with (1.11) and (1.14).

\section{Proposition 4.1}

Let $T>0, b_{1} \in\left[0 ; 1 / 2\left[\right.\right.$ and $u \in \mathbf{B}_{\lambda}^{\alpha}(T), f \in \mathbf{N}_{\lambda}^{\alpha, b_{1}}(T)$ satisfying

$$
\partial_{t} u+\partial_{x}^{3} u-\partial_{x}^{-1} \partial_{y}^{2} u=f
$$

on $[-T, T] \times \mathbb{R} \times \mathbb{T}_{\lambda}$.

Then $u \in \mathbf{F}_{\lambda}^{\alpha, b_{1}}(T)$ and

$$
\|u\|_{\mathbf{F}_{\lambda}^{\alpha, b_{1}}(T)} \lesssim\|u\|_{\mathbf{B}_{\lambda}^{\alpha}(T)}+\|f\|_{\mathbf{N}_{\lambda}^{\alpha, b_{1}}(T)}
$$

\section{Proof :}

This proposition is proved in [9] (see also [14]). We recall the proof here for completeness. 
Looking at the definition of $\mathbf{F}_{\lambda}^{\alpha, b_{1}}(T)(3.2), \mathbf{N}_{\lambda}^{\alpha, b_{1}}(T)(3.3)$ and $\mathbf{B}_{\lambda}^{\alpha}(T)$ (3.4), we have to prove that $\forall M>0$,

$$
\begin{array}{r}
\left\|P_{M} u\right\|_{F_{\lambda, M}^{b_{1}}(T)} \lesssim\left\|P_{M} u_{0}\right\|_{\mathbf{E}_{\lambda}^{0}}+\left\|P_{M} f\right\|_{N_{\lambda, M}^{b_{1}}(T)} \text { if } 0<M \leqslant 1 \\
\left\|P_{M} u\right\|_{F_{\lambda, M}^{b_{1}}(T)} \lesssim \sup _{t_{M} \in[-T, T]}\left\|P_{M} u\left(t_{M}\right)\right\|_{\mathbf{E}_{\lambda}^{0}}+\|\left. P_{M} f\right|_{N_{\lambda, M}^{b_{1}}(T)} \text { if } M>1
\end{array}
$$

Let $M>0$. As in [14, Proposition 2.9, p.14], we begin by constructing extensions $\widetilde{u}_{M}$ (respectively $\widetilde{f}_{M}$ ) of $P_{M} u$ (respectively $P_{M} f$ ) to $\mathbb{R}$, with a control on the boundary terms. To do so, we first define the smooth cutoff function

$$
m_{M}(t):=\left\{\begin{array}{l}
\chi_{(1 \vee M)^{-1} / 10}(t+T) \text { if } t<-T \\
1 \text { if } t \in[-T, T] \\
\chi_{(1 \vee M)^{-1} / 10}(t-T) \text { if } t>T
\end{array}\right.
$$

Next, we define $\widetilde{f}_{M}$ on $\mathbb{R}$ with

$$
\widetilde{f_{M}}(t):=m_{M}(t) f_{M}(t)
$$

where $f_{M}$ is an extension of $P_{M} f$ to $\mathbb{R}$ satisfying $\left\|f_{M}\right\|_{N_{\lambda, M}^{b_{1}}} \leqslant 2\left\|P_{M} f\right\|_{N_{\lambda, M}^{b_{1}}(T)}$.

So $\widetilde{f_{M}}$ is also an extension of $P_{M} f$, with supp $\widetilde{f_{M}} \subset\left[-T-(1 \vee M)^{-1} / 5, T+(1 \vee M)^{-1} / 5\right]$. From (4.1), we have that

$$
P_{M} u(t)=U(t) P_{M} u_{0}+\int_{0}^{t} U\left(t-t^{\prime}\right) P_{M} f\left(t^{\prime}\right) \mathrm{d} t^{\prime} \text { on }[-T, T]
$$

Thus we define $\widetilde{u_{M}}$ as

$$
\widetilde{u_{M}}(t):=m_{M}(t)\left\{U(t) P_{M} u_{0}+\int_{0}^{t} U\left(t-t^{\prime}\right) \widetilde{f_{M}}\left(t^{\prime}\right) \mathrm{d} t^{\prime}\right\}, t \in \mathbb{R}
$$

The choice of $\widetilde{f_{M}}$ and $\widetilde{u_{M}}$ is dictated from the necessity to control the boundary term. First using (3.15) with $m_{M}$ we have

$$
\left\|\widetilde{f_{M}}\right\|_{N_{\lambda, M}^{b_{1}}} \lesssim\left\|P_{M} f\right\|_{N_{\lambda, M}^{b_{1}}(T)}
$$

and $\widetilde{u_{M}}$ defines an extension of $P_{M} u$.

Moreover, if $t_{M} \notin[-T, T]$, from the choice of $m_{M}$, we can write $\chi_{(1 \vee M)^{-1}}\left(t-t_{M}\right) \widetilde{u_{M}}(t)=$ $\chi_{(1 \vee M)^{-1}}\left(t-\widetilde{t_{M}}\right) \chi_{(1 \vee M)^{-1}}\left(t-t_{M}\right) \widetilde{u_{M}}(t)$ for a $\widetilde{t_{M}} \in[-T, T]$. Then, using (3.10) and (3.11) we get

$$
\sup _{t_{M} \notin[-T, T]}\left\|\chi_{(1 \vee M)^{-1}}\left(t-t_{M}\right) \widetilde{u_{M}}||_{X_{\lambda, M}^{b_{1}}} \lesssim \sup _{\widetilde{t_{M}} \in[-T, T]}\right\| \chi_{(1 \vee M)^{-1}}\left(t-\widetilde{t_{M}}\right) \widetilde{u_{M}}||_{X_{\lambda, M}^{b_{1}}}
$$

Thus it suffices to prove

$$
\begin{aligned}
\sup _{t_{M} \in[-T, T]}\left\|p \cdot \mathcal{F}\left\{\chi\left(t-t_{M}\right) \widetilde{u_{M}}\right\}\right\|_{X_{\lambda, M}^{b_{1}}} \lesssim\left\|\widetilde{u_{M}}(0)\right\|_{\mathbf{E}_{\lambda}^{0}}+ \\
\sup _{\widetilde{t_{M}} \in \mathbb{R}}\left\|(\tau-\omega+i)^{-1} p \cdot \mathcal{F}\left\{\chi\left(t-\widetilde{t_{M}}\right) \widetilde{f_{M}}\right\}\right\|_{X_{\lambda, M}^{b_{1}}} \text { if } M \leqslant 1
\end{aligned}
$$


and

$$
\begin{aligned}
\sup _{t_{M} \in[-T, T]}\left\|p \cdot \mathcal{F}\left\{\chi_{M^{-1}}\left(t-t_{M}\right) \widetilde{u_{M}}\right\}\right\|_{X_{\lambda, M}^{b_{1}}} \lesssim \sup _{\widehat{t_{M}} \in[-T, T]}\left\|\widetilde{u_{M}}\left(\widehat{t_{M}}\right)\right\|_{\mathbf{E}_{\lambda}^{0}}+ \\
\sup _{\widetilde{t_{M}} \in \mathbb{R}}\left\|(\tau-\omega+i M)^{-1} p \cdot \mathcal{F}\left\{\chi_{M^{-1}}\left(t-\widetilde{t_{M}}\right) \widetilde{f_{M}}\right\}\right\|_{X_{\lambda, M}^{b_{1}}} \text { if } M>1
\end{aligned}
$$

Note that, since $m_{M} \equiv 1$ on $[-T, T]$ and $u$ is a solution of (4.1), for $t_{M} \in[-T, T]$, we have

$$
P_{M} u\left(t_{M}\right)=U\left(t_{M}\right) P_{M} u_{0}+\int_{0}^{t_{M}} U\left(t-t^{\prime}\right) \widetilde{f_{M}}\left(t^{\prime}\right) \mathrm{d} t^{\prime}
$$

and thus

$$
\widetilde{u_{M}}\left(t+t_{M}\right)=m_{M}\left(t+t_{M}\right)\left\{U(t) P_{M} u\left(t_{M}\right)+\int_{0}^{t} U\left(t-t^{\prime}\right) \widetilde{f_{M}}\left(t^{\prime}+t_{M}\right) \mathrm{d} t^{\prime}\right\}
$$

Finally, it suffices to prove that

$$
\left\|p \cdot \mathcal{F}\left\{\chi\left(t-t_{M}\right) m_{M}(t) U(t) P_{M} u_{0}\right\}\right\|_{X_{\lambda, M}^{b_{1}}} \lesssim \|\left.\widetilde{u_{M}}(0)\right|_{\mathbf{E}_{\lambda}^{0}}
$$

and

$$
\begin{aligned}
&\left\|p \cdot \mathcal{F}\left\{\chi\left(t-t_{M}\right) m_{M}(t) \int_{0}^{t} U\left(t-t^{\prime}\right) \widetilde{f_{M}}\left(t^{\prime}\right) \mathrm{d} t^{\prime}\right\}\right\|_{X_{\lambda, M}^{b_{1}}} \\
& \lesssim\left\|(\tau-\omega+i)^{-1} p \cdot \mathcal{F}\left\{\chi\left(t-t_{M}\right) \widetilde{f_{M}}\right\}\right\|_{X_{\lambda, M}^{b_{1}}}
\end{aligned}
$$

for the low-frequency part, and

$$
\left\|p \cdot \mathcal{F}\left\{\chi_{M^{-1}}(t) m_{M}\left(t+t_{M}\right) U(t) P_{M} u\left(t_{M}\right)\right\}\right\|_{X_{\lambda, M}^{b_{1}}} \lesssim\left\|\widetilde{u_{M}}\left(t_{M}\right)\right\|_{\mathbf{E}_{\lambda}^{0}}
$$

and

$$
\begin{aligned}
\| p \cdot \mathcal{F}\left\{\chi_{M^{-1}}(t) m_{M}\left(t+t_{M}\right)\right. & \left.\int_{0}^{t} U\left(t-t^{\prime}\right) \widetilde{f_{M}}\left(t_{M}+t^{\prime}\right) \mathrm{d} t^{\prime}\right\} \|_{X_{\lambda, M}^{b_{1}}} \\
& \lesssim\left\|(\tau-\omega+i M)^{-1} p \cdot \mathcal{F}\left\{\chi_{M^{-1}}\left(t-t_{M}\right) \widetilde{f_{M}}\right\}\right\|_{X_{\lambda, M}^{b_{1}}}
\end{aligned}
$$

for the high-frequency part.

To prove those estimates, we first notice that, since $t^{\prime} \in[0 ; t]$ and $t \in \operatorname{supp} \chi_{(1 \vee M)^{-1}}$, we can write $\widetilde{f_{M}}$ as

$$
\widetilde{f_{M}}\left(t_{M}+t^{\prime}\right)=\sum_{|n| \leqslant 100} f_{M, n}\left(t_{M}+t^{\prime}\right):=\sum_{|n| \leqslant 100} \gamma\left((1 \vee M) t^{\prime}-n\right) \widetilde{f_{M}}\left(t_{M}+t^{\prime}\right)
$$

where $\gamma: \mathbb{R} \rightarrow[0 ; 1]$ is a smooth partition of unity, satisfying supp $\gamma \subset[-1 ; 1]$ and for all $x \in \mathbb{R}$,

$$
\sum_{n \in \mathbb{Z}} \gamma(x-n)=1
$$


The second observation is that, for a fixed $t_{M}$, we have for the homogeneous term

$$
\left\|p \cdot \mathcal{F}\left\{\chi_{M^{-1}}(t) m_{M}\left(t+t_{M}\right) U(t) P_{M} u\left(t_{M}\right)\right\}\right\|_{X_{\lambda, M}^{b_{1}}} \lesssim\left\|m_{M} U(t) P_{M} u\left(t_{M}\right)\right\|_{F_{\lambda, M}^{b_{1}}}
$$

so we can remove the localization $m_{M}(t)$ thanks to (3.14), and similarly for the inhomogeneous term.

Computing the Fourier transform in the left-hand side of (4.6) and using the bound

$$
\left\|\rho_{K}(\tau-\omega) \mathrm{e}^{i t_{M}(\tau-\omega)} \widehat{\chi}(\tau-\omega)\right\|_{L_{\tau}^{2}} \lesssim\left\|\rho_{K}(\tau)\langle\tau\rangle^{-2}\right\|_{L^{2}} \lesssim K^{-3 / 2}
$$

since $\hat{\chi} \in \mathcal{S}(\mathbb{R})$, we then obtain

$$
\begin{aligned}
& \left\|p \cdot \mathcal{F}\left\{\chi\left(t-t_{M}\right) U(t) P_{M} u_{0}\right\}\right\|_{X_{\lambda, M}^{b_{1}}} \\
& \quad \lesssim \sum_{K \geqslant 1} K^{1 / 2} \beta_{M, K}^{b_{1}}\left\|\rho_{K}(\tau-\omega) p \cdot \mathrm{e}^{i t_{M}(\tau-\omega)} \widehat{\chi}(\tau-\omega) \widehat{P_{M} u_{0}}\right\| \|_{L^{2}}
\end{aligned}
$$

$$
\lesssim\left\|P_{M} u_{0}\right\|_{L^{2}}
$$

The proof of (4.8) is the same replacing the first bound by

$$
\left\|\rho_{K}(\tau-\omega) M^{-1}\left\langle M^{-1}(\tau-\omega)\right\rangle^{-2}\right\|_{L_{\tau}^{2}} \lesssim M^{-1} K^{1 / 2}\left(1 \vee M^{-1} K\right)^{-2}
$$

For (4.7) and (4.9), a computation gives first

$$
\begin{aligned}
\mathcal{F}\left\{\chi_{(1 \vee M)^{-1}}(t) \int_{0}^{t} U\left(t-t^{\prime}\right) f_{M, n}\left(t_{M}+t^{\prime}\right) \mathrm{d} t^{\prime}\right\}(\tau) & \\
=(1 \vee M)^{-1} \int_{\mathbb{R}} \frac{\widehat{\chi}\left((1 \vee M)^{-1}\left(\tau-\tau^{\prime}\right)\right)-\widehat{\chi}\left((1 \vee M)^{-1}(\tau-\omega)\right)}{i\left(\tau^{\prime}-\omega\right)} & \cdot \mathrm{e}^{i t_{M} \tau^{\prime} \mathcal{F}\left\{f_{M, n}\right\}\left(\tau^{\prime}\right) \mathrm{d} \tau^{\prime}}
\end{aligned}
$$

Now, we distinguish between two cases, wether $\left|\tau^{\prime}-\omega+i(1 \vee M)\right| \sim\left|\tau^{\prime}-\omega\right|$ or $\mid \tau^{\prime}-\omega+$ $i(1 \vee M) \mid \sim(1 \vee M)$.

First, if $\left|\tau^{\prime}-\omega\right| \gtrsim(1 \vee M)$, we have

$$
\begin{aligned}
& \left|\frac{\widehat{\chi}\left((1 \vee M)^{-1}\left(\tau-\tau^{\prime}\right)\right)-\widehat{\chi}\left((1 \vee M)^{-1}(\tau-\omega)\right)}{i\left(\tau^{\prime}-\omega\right)}\right| \\
& \quad \lesssim\left|\frac{\widehat{\chi}\left((1 \vee M)^{-1}\left(\tau-\tau^{\prime}\right)\right)}{i\left(\tau^{\prime}-\omega+i(1 \vee M)\right)}\right|+\left|\frac{\widehat{\chi}\left((1 \vee M)^{-1}(\tau-\omega)\right)}{i\left(\tau^{\prime}-\omega+i(1 \vee M)\right)}\right|
\end{aligned}
$$

Now if $\left|\tau^{\prime}-\omega\right| \lesssim(1 \vee M)$ we apply the mean value theorem to $\widehat{\chi}$ so that

$$
\widehat{\chi}\left((1 \vee M)^{-1}\left(\tau-\tau^{\prime}\right)\right)-\widehat{\chi}\left((1 \vee M)^{-1}(\tau-\omega)\right)=(1 \vee M)^{-1} \widehat{\chi}^{\prime}(\theta) \cdot\left(\tau^{\prime}-\omega\right)
$$

for a $\theta \in\left[\tau-\tau^{\prime} ; \tau-\omega\right]$. Thus we have

$$
\begin{aligned}
\left|\frac{\widehat{\chi}\left((1 \vee M)^{-1}\left(\tau-\tau^{\prime}\right)\right)-\widehat{\chi}\left((1 \vee M)^{-1}(\tau-\omega)\right)}{i\left(\tau^{\prime}-\omega\right)}\right| \lesssim(1 \vee M)^{-1}\left|\widehat{\chi}^{\prime}(\theta)\right| & \\
& \lesssim\left|\tau^{\prime}-\omega+i(1 \vee M)\right|^{-1}\left|\widehat{\chi}^{\prime}(\theta)\right|
\end{aligned}
$$


Finally, using the assumption on $\theta$ and that $\hat{\chi} \in \mathcal{S}(\mathbb{R})$, we have in both cases

$$
\begin{aligned}
\left|\frac{\widehat{\chi}\left((1 \vee M)^{-1}\left(\tau-\tau^{\prime}\right)\right)-\widehat{\chi}\left((1 \vee M)^{-1}(\tau-\omega)\right)}{i\left(\tau^{\prime}-\omega\right)}\right| \\
\qquad\left|\frac{\left\langle(1 \vee M)^{-1}\left(\tau-\tau^{\prime}\right)\right\rangle^{-4}}{\tau^{\prime}-\omega+i(1 \vee M)}\right|+\left|\frac{\left\langle(1 \vee M)^{-1}(\tau-\omega)\right\rangle^{-4}}{\tau^{\prime}-\omega+i(1 \vee M)}\right|
\end{aligned}
$$

Coming back to (4.7) and (4.9), the left-hand side can be split into

$$
\begin{aligned}
\sum_{|n| \leqslant 100} \| p & \cdot(1 \vee M)^{-1} \int_{\mathbb{R}}\left|\frac{\left\langle(1 \vee M)^{-1}\left(\tau-\tau^{\prime}\right)\right\rangle^{-4}}{\tau^{\prime}-\omega+i(1 \vee M)} \mathcal{F}\left\{f_{M, n}\right\}\left(\tau^{\prime}\right)\right| \mathrm{d} \tau^{\prime} \mid \|_{X_{\lambda, M}^{b_{1}}} \\
& +\sum_{|n| \leqslant 100}\left\|p \cdot(1 \vee M)^{-1} \int_{\mathbb{R}} \frac{\left\langle(1 \vee M)^{-1}(\tau-\omega)\right\rangle^{-4}}{\tau^{\prime}-\omega+i(1 \vee M)} \mathcal{F}\left\{f_{M, n}\right\}\left(\tau^{\prime}\right)\left|\mathrm{d} \tau^{\prime}\right|\right\|_{X_{\lambda, M}^{b_{1}}}
\end{aligned}
$$

The first term is handled with (3.10) and (3.11) with $K_{0}=(1 \vee M)$ and $\gamma=\mathcal{F}^{-1}\left\{\langle\cdot\rangle^{-4}\right\}$. This term is thus controled by

$$
\sup _{|n| \leqslant 100}\left\|p \cdot(\tau-\omega+i(1 \vee M))^{-1} \mathcal{F}\left\{f_{M, n}\right\}\right\|_{X_{\lambda, M}^{b_{1}}} \lesssim\left\|\widetilde{f_{M}}\right\|_{F_{\lambda, M}^{b_{1}}}
$$

where in the last step we have used that

$$
\gamma((1 \vee M) t-n)=\gamma((1 \vee M) t-n) \chi_{(1 \vee M)^{-1}}\left(t-(1 \vee M)^{-1} n\right)
$$

and (3.10)-(3.11) to get rid of $\gamma$.

It remains to treat the second term. By definition of the $X_{\lambda, M}^{b_{1}}$ norm, we can write it

$$
\begin{aligned}
& \sum_{K \geqslant 1} K^{1 / 2} \beta_{M, K}^{b_{1}} \| \rho_{K}(\tau-\omega) p \cdot(1 \vee M)^{-1} \\
& \cdot \int_{\mathbb{R}} \frac{\mid\left\langle(1 \vee M)^{-1}(\tau-\omega)\right\rangle^{-4}}{\tau^{\prime}-\omega+i(1 \vee M)} \mathcal{F}\left\{f_{M, n}\right\}\left(\tau^{\prime}\right)\left|\mathrm{d} \tau^{\prime}\right| \|_{L^{2}} \\
&=\sum_{K \geqslant 1} K^{1 / 2} \beta_{M, K}^{b_{1}}\|p \cdot\|\left(\tau^{\prime}-\omega+i(1 \vee M)\right)^{-1} \mathcal{F}\left\{f_{M, n}\right\} \|_{L_{\tau^{\prime}}^{1}} \\
& \cdot\left\|\rho_{K}(\tau-\omega)(1 \vee M)^{-1}\left\langle(1 \vee M)^{-1}(\tau-\omega)\right\rangle^{-4}\right\|_{L_{\tau}^{2}} \|_{L_{\xi, q}^{2}}
\end{aligned}
$$

Now, since

$$
\sum_{K \geqslant 1} K^{1 / 2} \beta_{M, K}^{b_{1}}(1 \vee M)^{-1}\left\langle(1 \vee M)^{-1} K\right\rangle^{-4}\left\|\rho_{K}\right\|_{L^{2}} \lesssim 1
$$

we can use (3.12) to bound the last term with

$$
\begin{aligned}
\|p \cdot\|\left(\tau^{\prime}-\omega+i(1 \vee M)\right)^{-1} \mathcal{F}\left\{f_{M, n}\right\} \|_{L_{\tau^{\prime}}^{1}} & \|_{L_{\xi, q}^{2}} \\
& \lesssim\left\|p \cdot\left(\tau^{\prime}-\omega+i(1 \vee M)\right)^{-1} \mathcal{F}\left\{f_{M, n}\right\}\right\|_{X_{\lambda, M}^{b_{1}}}
\end{aligned}
$$

which concludes the proof through the same argument than above. 
Proceeding in the same way at the $L^{2}$ level, we have also

\section{Proposition 4.2}

Let $T>0, b_{1} \in\left[0 ; 1 / 2\left[\right.\right.$ and $u \in{\overline{\mathbf{B}_{\lambda}}}^{b_{1}}(T), f \in{\overline{\mathbf{N}_{\lambda}}}^{b_{1}}(T)$ satisfying (4.1) on $[-T, T] \times \mathbb{R} \times \mathbb{T}_{\lambda}$.

Then

$$
\left.\|u\|\right|_{{\overline{\mathbf{F}_{\lambda}}}^{b_{1}}(T)} \lesssim \|\left. u\right|_{{\overline{\mathbf{B}_{\lambda}}}_{(T)}}+|| f||_{{\overline{\mathbf{N}_{\lambda}}}^{b_{1}}(T)}
$$

\section{Dyadic estimates}

As in the standard Bourgain method, we will need some bilinear estimates for functions localized in both their frequency and their modulatation. This section deals with estimating expressions under the form $\int f_{1} \star f_{2} \cdot f_{3}$ which will be usefull to prove the main bilinear estimate (1.12) as well as the energy estimate (1.13). The following lemma gives a first rough estimate :

\section{Lemma 5.1}

Let $f_{i} \in L^{2}\left(\mathbb{R}^{2} \times \lambda^{-1} \mathbb{Z}\right)$ be such that $\operatorname{supp} f_{i} \subset D_{\lambda, M_{i}, \leqslant K_{i}} \cap \mathbb{R}^{2} \times I_{i}$, with $M_{i} \in 2^{\mathbb{Z}}$, $K_{i} \in 2^{\mathbb{N}}$ and $I_{i} \subset \lambda^{-1} \mathbb{Z}, i=1,2,3$. Then

$$
\int_{\mathbb{R}^{2} \times \lambda^{-1} \mathbb{Z}} f_{1} \star f_{2} \cdot f_{3} \lesssim M_{\text {min }}^{1 / 2} K_{\min }^{1 / 2}|I|_{\text {min }}^{1 / 2} \prod_{i=1}^{3} \|\left. f_{i}\right|_{L^{2}}
$$

\section{Proof :}

The proof is the same as in [9, Lemma 5.1 (b)]. We just have to expand the convolution product in the left-hand side and then apply Cauchy-Schwarz inequality in the variable coresponding to the min : if, for example, $K_{1}=K_{\min }$, we have

$$
\int_{\mathbb{R}^{2} \times \lambda^{-1} \mathbb{Z}} f_{1} \star f_{2} \cdot f_{3}=\int_{\mathbb{R}^{2} \times \lambda^{-1} \mathbb{Z}} \int_{\mathbb{R}^{2} \times \lambda^{-1} \mathbb{Z}} f_{1}\left(\tau-\tau_{2}, \zeta-\zeta_{2}\right)
$$

$$
\cdot f_{2}\left(\tau_{2}, \zeta_{2}\right) f_{3}(\tau, \zeta) \mathrm{d} \tau_{2} \mathrm{~d} \tau \mathrm{d} \zeta_{2} \mathrm{~d} \zeta
$$

Using Cauchy-Schwarz inequality in $\tau$, the previous term is less than

$$
\int_{\mathbb{R} \times \lambda^{-1} \mathbb{Z}} \int_{\mathbb{R} \times \lambda^{-1} \mathbb{Z}}\left\|f_{3}(\zeta)\right\|_{L_{\tau}^{2}}\left\|\int_{\mathbb{R}} f_{1}\left(\tau-\tau_{2}, \zeta-\zeta_{2}\right) f_{2}\left(\tau_{2}, \zeta_{2}\right) \mathrm{d} \tau_{2}\right\|_{L_{\tau}^{2}} \mathrm{~d} \zeta_{2} \mathrm{~d} \zeta
$$

Next, a use of Young's inequality $L^{1} \times L^{2} \rightarrow L^{2}$ in $\tau$ gives the bound

$$
\int_{\mathbb{R} \times \lambda^{-1} \mathbb{Z}} \int_{\mathbb{R} \times \lambda^{-1} \mathbb{Z}}\left\|f_{3}(\zeta)\right\|_{L_{\tau}^{2}}\left\|\left.f_{2}\left(\zeta_{2}\right)\right|_{L_{\tau}^{2}}\right\| f_{1}\left(\zeta-\zeta_{2}\right) \|_{L_{\tau_{1}}^{1}} \mathrm{~d} \zeta_{2} \mathrm{~d} \zeta
$$

Finally, using again Cauchy-Schwarz inequality in $\tau_{1}$, the previous term is controlled with

$$
\int_{\mathbb{R} \times \lambda^{-1} \mathbb{Z}} \int_{\mathbb{R} \times \lambda^{-1} \mathbb{Z}}\left\|f_{3}(\zeta)\right\|_{L_{\tau}^{2}}\left\|f_{2}\left(\zeta_{2}\right)\right\|_{L_{\tau}^{2}} K_{1}^{1 / 2}\left\|f_{1}\left(\zeta-\zeta_{2}\right)\right\|_{L_{\tau_{1}}^{2}} \mathrm{~d} \zeta_{2} \mathrm{~d} \zeta
$$

We get (5.1) when proceeding similarly for the integrals in $\xi$ and $q$. 


\subsection{Localized Strichartz estimates}

The purpose of this subsection is to improve (5.1). All the estimates we need are already used in [19] in the context of the KP-II equation. We briefly recall the outline of the proof here for the sake of completeness.

First, we are going to use the following easy lemmas :

\section{Lemma 5.2}

Let $\Lambda \subset \mathbb{R} \times \lambda^{-1} \mathbb{Z}$. We assume that the projection of $\Lambda$ on the $\xi$ axis is contained in an interval $I \subset \mathbb{R}$. Moreover, we assume that the measure of the $q$-sections of $\Lambda$ (that is the sets $\left\{q \in \lambda^{-1} \mathbb{Z},\left(\xi_{0}, q\right) \in \Lambda\right\}$ for a fixed $\left.\xi_{0}\right)$ is uniformly (in $\xi_{0}$ ) bounded by a constant $C$. Then we have

$$
|\Lambda| \leqslant C|I|
$$

\section{Proof :}

The proof is immediate : by definition

$$
|\Lambda|=\int_{I}\left(\int \mathbb{1}_{\Lambda}(\xi, q)(\mathrm{d} q)_{\lambda}\right) \mathrm{d} \xi \leqslant \int_{I} C \mathrm{~d} \xi=C|I|
$$

\section{Lemma 5.3}

Let $I, J$ be two intervals in $\mathbb{R}$, and let $\varphi: I \rightarrow \mathbb{R}$ be a $\mathcal{C}^{1}$ function with $\inf _{\xi \in J}\left|\varphi^{\prime}(\xi)\right|>0$. Then

$$
|\{x \in J, \varphi(x) \in I\}| \leqslant \frac{|I|}{\inf _{\xi \in J}\left|\varphi^{\prime}(\xi)\right|}
$$

and

$$
\left|\left\{q \in J \cap \lambda^{-1} \mathbb{Z}, \varphi(q) \in I\right\}\right| \lesssim\left\langle\frac{|I|}{\inf _{\xi \in J}\left|\varphi^{\prime}(\xi)\right|}\right\rangle
$$

\section{Proof :}

Let us define

$$
\mathcal{J}:=\{x \in J, \varphi(x) \in I\}
$$

and

$$
\mathcal{J}_{\lambda}:=\left\{q \in J \cap \lambda^{-1} \mathbb{Z}, \varphi(q) \in I\right\}
$$

We just have to use the mean value theorem and write

$$
|\mathcal{J}|=\sup _{x_{1}, x_{2} \in \mathcal{J}}\left|x_{2}-x_{1}\right|=\sup _{x_{1}, x_{2} \in \mathcal{J}} \frac{\left|\varphi\left(x_{2}\right)-\varphi\left(x_{1}\right)\right|}{\left|\varphi^{\prime}(\theta)\right|}
$$

for a $\theta \in\left[x_{1} ; x_{2}\right]$, and (5.3) follows since $\sup _{x_{1}, x_{2} \in \mathcal{J}}\left|\varphi\left(x_{2}\right)-\varphi\left(x_{1}\right)\right| \leqslant|I|$ by definition of $\mathcal{J}$. The proof of (5.4) is the same, using that

$$
\left|\mathcal{J}_{\lambda}\right| \leqslant \lambda^{-1}+\sup _{q_{1}, q_{2} \in \mathcal{J}_{\lambda}}\left|q_{2}-q_{1}\right|
$$

by definition of $(\mathrm{d} q)_{\lambda}$. 


\section{Lemma 5.4}

Let $a \neq 0, b, c$ be real numbers and $I \subset \mathbb{R}$ a bounded interval. Then

$$
\left|\left\{x \in \mathbb{R}, a x^{2}+b x+c \in I\right\}\right| \lesssim \frac{|I|^{1 / 2}}{|a|^{1 / 2}}
$$

and

$$
\left|\left\{q \in \lambda^{-1} \mathbb{Z}, a q^{2}+b q+c \in I\right\}\right| \lesssim\left\langle\frac{|I|^{1 / 2}}{|a|^{1 / 2}}\right\rangle
$$

Proof :

We begin by proving (5.5). By the linear change of variable $x \mapsto x+b /(2 a)$ it suffices to evaluate

$$
\left|\left\{y \in \mathbb{R}, a y^{2} \in \widetilde{I}\right\}\right| \text { with } \widetilde{I}=I+b^{2} /(4 a)-c,|\widetilde{I}|=|I|
$$

Writing $\varepsilon:=\operatorname{sign}(a)$, the measure of the previous set is

$$
\int_{\mathbb{R}} \mathbb{1}_{\widetilde{I}}\left(a y^{2}\right) \mathrm{d} y=|a|^{-1 / 2} \int_{\mathbb{R}} \mathbb{1}_{\widetilde{I}}\left(x^{2}\right) \mathrm{d} x
$$

- If $0 \notin \varepsilon \widetilde{I}$, by symmetry we may assume $\varepsilon \widetilde{I} \subset \mathbb{R}_{+}^{*}$ and write $\varepsilon \widetilde{I}=\left[x_{1} ; x_{2}\right]$ with $0<x_{1}<$ $x_{2}$. Then an easy computation gives

$$
\begin{aligned}
\left|\left\{y \in \mathbb{R}, a y^{2} \in \widetilde{I}\right\}\right|=|a|^{-1 / 2} \int_{\mathbb{R}} \mathbb{1}_{\left[x_{1} ; x_{2}\right]}\left(x^{2}\right) \mathrm{d} x=|a|^{-1 / 2} \int_{\mathbb{R}} \mathbb{1}_{\left[x_{1} ; x_{2}\right]}(y) \frac{\mathrm{d} y}{2 \sqrt{y}} \\
=|a|^{-1 / 2}[\sqrt{y}]_{x_{1}}^{x_{2}}=|a|^{-1 / 2}\left(\sqrt{x_{2}}-\sqrt{x_{1}}\right) \leqslant|a|^{-1 / 2}|I|^{1 / 2}
\end{aligned}
$$

- If $0 \in \varepsilon \widetilde{I}$ : defining $I^{+}:=(\varepsilon \widetilde{I} \cup-\varepsilon \widetilde{I}) \cap \mathbb{R}^{+}=\left[0 ; x_{2}\right]$ we have

$$
\left|\left\{y \in \mathbb{R}, a y^{2} \in \widetilde{I}\right\}\right| \leqslant 2|a|^{-1 / 2} \int_{\mathbb{R}} \mathbb{1}_{I^{+}}\left(x^{2}\right) \mathrm{d} x=2|a|^{-1 / 2} \sqrt{x_{2}} \lesssim|a|^{-1 / 2}|I|^{1 / 2}
$$

The proof of (5.6) follows from (5.5) through the same argument as in the proof of (5.4). 
The main estimates of this section are the following.

\section{Proposition 5.5 (Dyadic $L^{4}-L^{2}$ Strichartz estimate)}

Let $M_{1}, M_{2}, M_{3} \in 2^{\mathbb{Z}}, K_{1}, K_{2}, K_{3} \in 2^{\mathbb{N}}$ and let $u_{i} \in L^{2}\left(\mathbb{R}^{2} \times \lambda^{-1} \mathbb{Z}\right), i=1,2$, be such that $\operatorname{supp}\left(u_{i}\right) \subset D_{\lambda, M_{i}, \leqslant K_{i}}$. Then

$$
\begin{aligned}
&\left\|\mathbb{1}_{D_{\lambda, M_{3}, \leqslant K_{3}}} \cdot u_{1} \star u_{2}\right\|_{L^{2}} \lesssim\left(K_{1} \wedge K_{2}\right)^{1 / 2} M_{\min }^{1 / 2} \\
& \cdot\left\langle\left(K_{1} \vee K_{2}\right)^{1 / 4}\left(M_{1} \wedge M_{2}\right)^{1 / 4}\right\rangle\left\|u_{1}\right\|_{L^{2}}\left\|u_{2}\right\|_{L^{2}}
\end{aligned}
$$

Moreover, if we are in the regime $K_{\max } \leqslant 10^{-10} M_{1} M_{2} M_{3}$ then

$$
\begin{aligned}
\left\|\mathbb{1}_{D_{\lambda, M_{3}, \leqslant K_{3}}} \cdot u_{1} \star u_{2}\right\|_{L^{2}} \lesssim\left(K_{1} \wedge K_{2}\right)^{1 / 2} M_{\text {min }}^{1 / 2} & \\
\cdot & \left\langle\left(K_{1} \vee K_{2}\right)^{1 / 2} M_{\text {max }}^{-1 / 2}\right\rangle\left\|u_{1}\right\|_{L^{2}}\left\|u_{2}\right\|_{L^{2}}
\end{aligned}
$$

\section{Proof :}

These estimates are proven in [19, Proposition 2.1 \& Corollaire 2.9] and [22, Theorem 2.1, p.456-458] for functions $f_{i} \in L^{2}\left(\mathbb{R}^{2} \times \mathbb{Z}\right)$ but with a slightly different support condition : the localization with respect to the modulations is done for the symbol of the linear operator associated with the KP-II equation (i.e $\left.\widetilde{\omega}(\xi, q)=\xi^{3}-q^{2} / \xi\right)$, and the fifth-order KP-I equation $\left(\omega^{5 \text { th }}(m, \eta)=-m^{5}-\eta^{2} / m\right)$ respectively. As a matter of fact, the proof only uses the form of the expression $\left(q_{1} / \xi_{1}-q_{2} / \xi_{2}\right)$ but does not take into account its sign within the resonnant function. Thus we can obtain the similar estimates for the KP-I equation. Let us recall the main steps in proving these estimates : first, split $u_{1}$ and $u_{2}$ depending on the value of $\xi_{i}$ on an $M_{3}$ scale

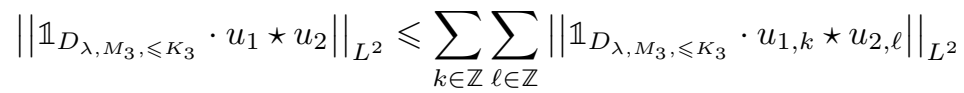

with

$$
u_{i, j}:=\mathbb{1}_{\left[j M_{3},(j+1) M_{3}\right]}\left(\xi_{i}\right) u_{i}
$$

The conditions $|\xi| \sim M_{3}, \xi_{1} \in\left[k M_{3},(k+1) M_{3}\right]$ and $\xi-\xi_{1} \in\left[\ell M_{3} ;(\ell+1) M_{3}\right]$ require $\ell \in[-k-c ;-k+c]$ for an absolute constant $c>0$. Thus we have to get estimates for functions $u_{i}$ supported in $D_{\lambda, M_{i}, K_{i}} \cap\left\{\xi_{i} \in I_{i}\right\}$ for some intervals $I_{i}$.

Moreover, we may assume $\xi_{i} \geqslant 0$ on supp $u_{i}$ (see [22, p.460]). This is crucial as $\xi \sim \xi_{1} \vee\left(\xi-\xi_{1}\right)$ in this case.

Squaring the left-hand side, it then suffices to evaluate

$$
\int_{\mathbb{R} \times \mathbb{R}_{+} \times \lambda^{-1} \mathbb{Z}} \mid \int_{\mathbb{R} \times \mathbb{R}_{+} \times \lambda^{-1} \mathbb{Z}} \mathbb{1}_{\left.D_{\lambda, M_{3}, \leqslant K_{3}} \cdot u_{1}\left(\tau_{1}, \zeta_{1}\right) u_{2}\left(\tau-\tau_{1}, \zeta-\zeta_{1}\right) \mathrm{d} \tau_{1} \mathrm{~d} \xi_{1}\left(\mathrm{~d} q_{1}\right)_{\lambda}\right|^{2}}^{\mathrm{d} \tau \mathrm{d} \xi(\mathrm{d} q)_{\lambda}}
$$

Using Cauchy-Schwarz inequality, the integral above is controled by

$$
\sup _{\tau, \xi \geqslant 0, q \in D_{\lambda, M_{3}, \leqslant K_{3}}}\left|A_{\tau, \xi, q}\right| \cdot\left\|u_{1}\right\|_{L^{2}}^{2}\left\|u_{2}\right\|_{L^{2}}^{2}
$$


where $A_{\tau, \xi, q}$ is defined as

$$
\begin{aligned}
A_{\tau, \xi, q}:=\left\{\left(\tau_{1}, \zeta_{1}\right) \in \mathbb{R} \times \mathbb{R}_{+} \times \lambda^{-1} \mathbb{Z}, \xi_{1} \in I_{1}, \xi-\xi_{1} \in I_{2}, 0 \leqslant \xi_{1} \sim M_{1},\right. \\
\left.0 \leqslant \xi-\xi_{1} \sim M_{2},\left\langle\tau_{1}-\omega\left(\zeta_{1}\right)\right\rangle \lesssim K_{1},\left\langle\tau-\tau_{1}-\omega\left(\zeta-\zeta_{1}\right)\right\rangle \lesssim K_{2}\right\}
\end{aligned}
$$

Using the triangle inequality in $\tau_{1}$, we get the bound

$$
\left|A_{\tau, \xi, q}\right| \lesssim\left(K_{1} \wedge K_{2}\right)\left|B_{\tau, \xi, q}\right|
$$

where $B_{\tau, \xi, q}$ is defined as

$$
\begin{aligned}
B_{\tau, \xi, q}:=\left\{\zeta_{1} \in \mathbb{R}_{+} \times \lambda^{-1} \mathbb{Z}, \xi_{1} \in I_{1}, \xi-\xi_{1} \in I_{2}, 0 \leqslant \xi_{1} \sim M_{1},\right. \\
\left.0 \leqslant \xi-\xi_{1} \sim M_{2},\left\langle\tau-\omega(\zeta)-\Omega\left(\zeta_{1}, \zeta-\zeta_{1},-\zeta\right)\right\rangle \lesssim\left(K_{1} \vee K_{2}\right)\right\}
\end{aligned}
$$

where $\Omega$ is the resonant function for (1.6), defined on the hyperplane $\zeta_{1}+\zeta_{2}+\zeta_{3}=0$ :

$$
\begin{aligned}
\Omega\left(\zeta_{1}, \zeta_{2}, \zeta_{3}\right):=\omega\left(\zeta_{1}\right)+\omega\left(\zeta_{2}\right) & +\omega\left(\zeta_{3}\right) \\
= & -3 \xi_{1} \xi_{2} \xi_{3}+\frac{\left(\xi_{1} q_{2}-\xi_{2} q_{1}\right)^{2}}{\xi_{1} \xi_{2} \xi_{3}} \\
& =-\frac{\xi_{1} \xi_{2}}{\xi_{1}+\xi_{2}}\left\{\left(\sqrt{3} \xi_{1}+\sqrt{3} \xi_{2}\right)^{2}-\left(\frac{q_{1}}{\xi_{1}}-\frac{q_{2}}{\xi_{2}}\right)^{2}\right\}
\end{aligned}
$$

Now, (5.7) follows directly from applying lemma 5.2 and (5.6) to $B_{\tau, \xi, q}$ since its projection on the $\xi_{1}$ axis is controled by $\left|I_{1}\right| \wedge\left|I_{2}\right|$, whereas for a fixed $\xi_{1}$, the cardinal of the $q_{1}$-section is estimated by $\left\langle\left(K_{1} \vee K_{2}\right)^{1 / 2}\left(M_{1} \wedge M_{2}\right)^{1 / 2}\right\rangle$ using (5.6) as $\tau-\omega(\zeta)-\Omega\left(\zeta_{1}, \zeta-\zeta_{1},-\zeta\right)$ is a polynomial of second order in $q_{1}$, with a dominant coefficient $\sim\left(M_{1} \wedge M_{2}\right)^{-1}$. Thus

$$
\left|B_{\tau, \xi, q}\right| \lesssim\left(\left|I_{1}\right| \wedge\left|I_{2}\right|\right)\left\langle\left(K_{1} \vee K_{2}\right)^{1 / 2}\left(M_{1} \wedge M_{2}\right)^{1 / 2}\right\rangle
$$

which gives the estimate (5.7) when applied with $I_{1}=\left[k M_{3} ;(k+1) M_{3}\right] \cap \mathfrak{I}_{M_{1}}$ and $I_{2}=$ $\left[\ell M_{3},(\ell+1) M_{3}\right] \cap \mathfrak{I}_{M_{2}}$ and using Cauchy-Schwarz inequality to sum over $k \in \mathbb{Z}$.

In the case $K_{\max } \leqslant 10^{-10} M_{1} M_{2} M_{3}$, we compute

$$
\left|\frac{\partial \Omega}{\partial q_{1}}\right|=2\left|\frac{q_{1}}{\xi_{1}}-\frac{q-q_{1}}{\xi-\xi_{1}}\right|=2\left\{\frac{\xi}{\xi_{1}\left(\xi-\xi_{1}\right)}\left(\Omega+3 \xi_{1}\left(\xi-\xi_{1}\right) \xi\right)\right\}^{1 / 2}
$$

Thus, from the condition $|\Omega| \lesssim K_{\max } \leqslant 10^{-10} M_{1} M_{2} M_{3}$ we get

$$
\left|\frac{\partial \Omega}{\partial q_{1}}\right| \gtrsim\left|\frac{\xi}{\xi_{1}\left(\xi-\xi_{1}\right)} \cdot \xi_{1}\left(\xi-\xi_{1}\right) \xi\right|^{1 / 2} \sim M_{\max }
$$

At last, we can estimate $\left|B_{\tau, \xi, q}\right|$ in this regime by using (5.4) instead of (5.6), which gives the final bound

$$
\left|B_{\tau, \xi, q}\right| \lesssim\left(\left|I_{1}\right| \wedge\left|I_{2}\right|\right)\left\langle\left(K_{1} \vee K_{2}\right) M_{\max }^{-1}\right\rangle
$$

and (5.8) follows through the same argument as for (5.7).

Remark 5.6. The estimate (5.7) is rather crude, yet sufficient for our purpose. (5.8) is better than (5.10) below in the regime $K_{\max } \lesssim M_{1} M_{2} M_{3}, M_{\min } \leqslant 1$. Thus we do not need to use some function spaces with a special low-frequency structure as in [9] to deal with the difference equation, therefore we get a stronger uniqueness criterion. Note that we can perform the same argument in $\mathbb{R}^{2}$. 


\subsection{Dyadic bilinear estimates}

We are now looking to improve $(5.8)$ in the case $M_{m i n} \geqslant 1$. We mainly follow $[25$, Lemme 3.1]. However, in our situation the frequency for the $x$ variable lives in $\mathbb{R}$ and not in $\mathbb{Z}$, and thus the worst case of [25, Lemma 3.1] (when $K_{\text {med }} \lesssim M_{\max } M_{\min }$ ) is avoided. So, using that this frequency is allowed to vary in very small intervals, we are able to recover the same result as in [9, Lemme 5.1(a)]. Again, we will crucially use lemmas 5.2 and 5.3.

\section{Proposition 5.7}

Let $M_{i}, K_{i} \in 2^{\mathbb{N}}$ and $f_{i}: \mathbb{R}^{2} \times \lambda^{-1} \mathbb{Z} \rightarrow \mathbb{R}^{+}, i=1,2,3$, be such that $f_{i} \in L^{2}\left(\mathbb{R}^{2} \times \lambda^{-1} \mathbb{Z}\right)$ with $\operatorname{supp} f_{i} \subset D_{\lambda, M_{i}, \leqslant K_{i}}$.

If $K_{\text {max }} \leqslant 10^{-10} M_{1} M_{2} M_{3}$ and $K_{\text {med }} \gtrsim M_{\text {max }}$, then

$$
\int_{\mathbb{R}^{2} \times \lambda^{-1} \mathbb{Z}} f_{1} \star f_{2} \cdot f_{3} \mathrm{~d} \tau \mathrm{d} \xi(\mathrm{d} q)_{\lambda} \lesssim\left(\frac{K_{1} K_{2} K_{3}}{M_{1} M_{2} M_{3}}\right)^{1 / 2}\left\|f_{1}\right\|_{L^{2}}\left\|f_{2}\right\|_{L^{2}}\left\|f_{3}\right\|_{L^{2}}
$$

\section{Proof :}

We begin as in [9, Lemma 5.1(a)]. Defining

$$
\mathcal{I}\left(f_{1}, f_{2}, f_{3}\right):=\int_{\mathbb{R}^{2} \times \lambda^{-1} \mathbb{Z}} f_{1} \star f_{2} \cdot f_{3} \mathrm{~d} \tau \mathrm{d} \xi(\mathrm{d} q)_{\lambda}
$$

we observe that

$$
\mathcal{I}\left(f_{1}, f_{2}, f_{3}\right)=\mathcal{I}\left(\tilde{f}_{1}, f_{3}, f_{2}\right)=\mathcal{I}\left(\tilde{f}_{2}, f_{3}, f_{1}\right)
$$

where we define $\widetilde{f}(x):=f(-x)$. Thus, as $\|\widetilde{f}\|_{L^{2}}=\|f\|_{L^{2}}$, up to replacing $f_{i}$ by $\tilde{f}_{i}$, we may assume $K_{1} \leqslant K_{2} \leqslant K_{3}$.

Moreover, since the expression is symmetrical in $f_{1}, f_{2}$ we can assume $M_{2} \leqslant M_{1}$.

We first write

$$
\begin{aligned}
\mathcal{I}\left(f_{1}, f_{2}, f_{3}\right) & =\int_{\mathbb{R}^{2} \times \lambda^{-1} \mathbb{Z}} f_{1} \star f_{2} \cdot f_{3} \mathrm{~d} \tau \mathrm{d} \xi(\mathrm{d} q)_{\lambda} \\
& =\int_{\mathbb{R}^{2} \times \lambda^{-1} \mathbb{Z}} \int_{\mathbb{R} \times \mathbb{R}^{+} \times \lambda^{-1} \mathbb{Z}} f_{1}\left(\tau_{1}, \zeta_{1}\right) f_{2}\left(\tau_{2}, \zeta_{2}\right) f_{3}\left(\tau_{1}+\tau_{2}, \zeta_{1}+\zeta_{2}\right)
\end{aligned}
$$

$\mathrm{d} \tau_{1} \mathrm{~d} \tau_{2} \mathrm{~d} \zeta_{1} \mathrm{~d} \zeta_{2}$

Defining $f_{i}^{\#}(\theta, \zeta):=f_{i}(\theta+\omega(\zeta), \zeta)$ we get $\left\|f_{i}^{\#}\right\|_{L^{2}}=\left\|f_{i}\right\|_{L^{2}}$ and $\operatorname{supp} f_{i}^{\#} \subset\left\{|\theta| \lesssim K_{i},|\xi| \sim\right.$ $M_{i}$. Changing of variables, we have

$$
\begin{aligned}
\mathcal{I}\left(f_{1}, f_{2}, f_{3}\right)=\int_{\mathbb{R}^{2} \times \lambda^{-1} \mathbb{Z}} \int_{\mathbb{R}^{2} \times \lambda^{-1} \mathbb{Z}} f_{1}^{\#}\left(\theta_{1}, \zeta_{1}\right) f_{2}^{\#}\left(\theta_{2}, \zeta_{2}\right) \\
\cdot f_{3}^{\#}\left(\theta_{1}+\theta_{2}+\Omega\left(\zeta_{1}, \zeta_{2},-\zeta_{1}-\zeta_{2}\right), \zeta_{1}+\zeta_{2}\right) \mathrm{d} \theta_{1} \mathrm{~d} \theta_{2} \mathrm{~d} \zeta_{1} \mathrm{~d} \zeta_{2}
\end{aligned}
$$

where the resonant function

$$
\Omega\left(\zeta_{1}, \zeta_{2},-\zeta_{1}-\zeta_{2}\right)=-\frac{\xi_{1} \xi_{2}}{\xi_{1}+\xi_{2}}\left\{\sqrt{3}\left|\xi_{1}+\xi_{2}\right|+\left|\frac{q_{1}}{\xi_{1}}-\frac{q_{2}}{\xi_{2}}\right|\right\}\left\{\sqrt{3}\left|\xi_{1}+\xi_{2}\right|-\left|\frac{q_{1}}{\xi_{1}}-\frac{q_{2}}{\xi_{2}}\right|\right\}
$$

has been defined in (5.9) in the previous proposition. 
Thus

$$
\begin{aligned}
\mathcal{I}\left(f_{1}, f_{2}, f_{3}\right)=\int_{A} f_{1}^{\#}\left(\theta_{1}, \zeta_{1}\right) f_{2}^{\#}\left(\theta_{2}, \zeta_{2}\right) & \\
& \cdot f_{3}^{\#}\left(\theta_{1}+\theta_{2}+\Omega\left(\zeta_{1}, \zeta_{2},-\zeta_{1}-\zeta_{2}\right), \zeta_{1}+\zeta_{2}\right) \mathrm{d} \theta_{1} \mathrm{~d} \theta_{2} \mathrm{~d} \zeta_{1} \mathrm{~d} \zeta_{2}
\end{aligned}
$$

with

$$
\begin{array}{r}
A:=\left\{\left(\theta_{1}, \zeta_{1}, \theta_{2}, \zeta_{2}\right) \in\left(\mathbb{R}^{2} \times \lambda^{-1} \mathbb{Z}\right)^{2},\left|\xi_{i}\right| \sim M_{i},\left|\xi_{1}+\xi_{2}\right| \sim M_{3},\left|\theta_{i}\right| \lesssim K_{i},\right. \\
\left.\left|\theta_{1}+\theta_{2}+\Omega\left(\zeta_{1}, \zeta_{2},-\zeta_{1}-\zeta_{2}\right)\right| \lesssim K_{3}, \quad i=1,2\right\}
\end{array}
$$

We can decompose $A \subset I_{\leqslant K_{1}} \times I_{\leqslant K_{2}} \times B$ with $B$ defined as

$$
B:=\left\{\left(\zeta_{1}, \zeta_{2}\right) \in\left(\mathbb{R} \times \lambda^{-1} \mathbb{Z}\right)^{2},\left|\xi_{i}\right| \sim M_{i}, i=1,2,\left|\xi_{1}+\xi_{2}\right| \sim M_{3},\right.
$$$$
\left.|\Omega| \lesssim K_{3}\right\}
$$

We can further split

$$
B=\bigsqcup_{|\ell| \lesssim K_{3} / K_{2}} B_{\ell}
$$

with

$$
B_{\ell}:=\left\{\left(\zeta_{1}, \zeta_{2}\right) \in B, \Omega \in\left[\ell K_{2} ;(\ell+1) K_{2}\right]\right\}
$$

and as well for $f_{3}$ :

$$
f_{3}^{\#}=\sum_{|\ell| \lesssim K_{3} / K_{2}} f_{3, \ell}^{\#} \text { with } f_{3, \ell}^{\#}(\theta, \xi, q):=\mathbb{1}_{\left[\ell K_{2},(\ell+1) K_{2}\right]}(\theta) f_{3}^{\#}(\theta, \xi, q)
$$

Next, using Cauchy-Schwarz inequality in $\theta_{2}$ then $\theta_{1}$, we obtain

$$
\begin{aligned}
& \mathcal{I}\left(f_{1}, f_{2}, f_{3}\right) \leqslant \sum_{\substack{|\ell| \lesssim K_{3} / K_{2}\\
}} \int_{I_{\leqslant K_{1}} \times B_{\ell}}\left|f_{1}^{\#}\left(\theta_{1}, \xi_{1}, q_{1}\right)\right|\left\|f_{2}^{\#}\left(\theta_{2}, \xi_{2}, q_{2}\right)\right\|_{L_{\theta_{2}}^{2}} \\
& \| f_{3, \ell}^{\#}\left(\theta_{1}+\theta_{2}+\Omega, \xi_{1}+\xi_{2}, q_{1}+q_{2}\right)||_{L_{\theta_{2}}^{2}} \mathrm{~d} \theta_{1} \mathrm{~d} \xi_{1} \mathrm{~d} \xi_{2}\left(\mathrm{~d} q_{1}\right)_{\lambda}\left(\mathrm{d} q_{2}\right)_{\lambda} \\
& \lesssim K_{1}^{1 / 2} \sum_{|\ell| \lesssim K_{3} / K_{2}} \int_{B_{\ell}}\left\|f_{1}^{\#}\left(\theta_{1}, \xi_{1}, q_{1}\right)||_{L_{\theta_{1}}^{2}}\right\| f_{2}^{\#}\left(\theta_{2}, \xi_{2}, q_{2}\right) \|_{L_{\theta_{2}}^{2}} \\
& \cdot\left\|f_{3, \ell}^{\#}\left(\theta, \xi_{1}+\xi_{2}, q_{1}+q_{2}\right)\right\|_{L_{\theta}^{2}} \mathrm{~d} \xi_{1} \mathrm{~d} \xi_{2}\left(\mathrm{~d} q_{1}\right)_{\lambda}\left(\mathrm{d} q_{2}\right)_{\lambda}
\end{aligned}
$$

This allows us to work with functions depending on $\left(\xi_{1}, q_{1}\right),\left(\xi_{2}, q_{2}\right)$ only, loosing just a factor $K_{1}^{1 / 2}$ in the process. The informations $|\Omega| \lesssim K_{3}$ and $\operatorname{supp} f_{3} \subset I_{\leqslant K_{3}} \times \mathfrak{I}_{M_{3}} \times \lambda^{-1} \mathbb{Z}$ have been kept in the decomposition on $\ell$ of $B$ and $f_{3}^{\#}$.

Finally, defining

$$
g_{i}\left(\xi_{i}, q_{i}\right):=\left\|f_{i}^{\#}\left(\theta_{i}, \xi_{i}, q_{i}\right)\right\|_{L_{\theta_{i}}^{2}}, i=1,2 \text { and } g_{3, \ell}(\xi, q):=\left\|f_{3, \ell}^{\#}(\theta, \xi, q)\right\|_{L_{\theta}^{2}}
$$

and writing

$$
J_{\ell}\left(g_{1}, g_{2}, g_{3, \ell}\right):=\int_{B_{\ell}} g_{1}\left(\xi_{1}, q_{1}\right) g_{2}\left(\xi_{2}, q_{2}\right) g_{3, \ell}\left(\xi_{1}+\xi_{2}, q_{1}+q_{2}\right) \mathrm{d} \xi_{1} \mathrm{~d} \xi_{2}\left(\mathrm{~d} q_{1}\right)_{\lambda}\left(\mathrm{d} q_{2}\right)_{\lambda}
$$


it suffices to prove that

$$
J:=\sum_{\ell} J_{\ell}\left(g_{1}, g_{2}, g_{3, \ell}\right) \lesssim\left(\frac{K_{2} K_{3}}{M_{1} M_{2} M_{3}}\right)^{1 / 2}\left\|g_{1}\right\|_{L_{\xi_{1}, q_{1}}^{2}}\left\|g_{2}\right\|_{L_{\xi_{2}, q_{2}}^{2}}\left\{\sum_{\ell}\left\|g_{3, \ell}\right\|_{L_{\xi, q}^{2}}^{2}\right\}^{1 / 2}
$$

As we are in the regime $K_{\max } \lesssim M_{1} M_{2} M_{3}, \Omega$ is close to zero. Since $q_{i} \in \lambda^{-1} \mathbb{Z}$, we cannot just make a change of variables as in [9, Lemme 5.1(a)]. Thus, to take into account that $\left(\sqrt{3} \xi_{1}+\sqrt{3} \xi_{2}\right)^{2} \sim\left(\frac{q_{1}}{\xi_{1}}-\frac{q_{2}}{\xi_{2}}\right)^{2}$, we split $B_{\ell}$ depending on the values of $q_{1}$ and $q_{2}$.

First, as in [9, Lemma 5.1(a)], we can split

$$
B_{\ell}:=B_{\ell}^{++} \sqcup B_{\ell}^{+-} \sqcup B_{\ell}^{-+} \sqcup B_{\ell}^{--}
$$

with

$$
B_{\ell}^{\varepsilon_{1}, \varepsilon_{2}}:=\left\{\left(\xi_{1}, q_{1}\right),\left(\xi_{2}, q_{2}\right) \in B_{\ell}, \operatorname{sign}\left(\xi_{1}+\xi_{2}\right)=\varepsilon_{1}, \operatorname{sign}\left(\frac{q_{1}}{\xi_{1}}-\frac{q_{2}}{\xi_{2}}\right)=\varepsilon_{2}\right\}
$$

where $\varepsilon_{i} \in\{ \pm 1\}$.

Since the transformations $\left(\xi_{1}, q_{1}\right),\left(\xi_{2}, q_{2}\right) \mapsto\left(\varepsilon_{1} \xi_{1}, \varepsilon_{2} q_{2}\right),\left(\varepsilon_{1} \xi_{1}, \varepsilon_{2} q_{2}\right)$ maps $B_{\ell}^{\varepsilon_{1}, \varepsilon_{2}}$ to $B_{\ell}^{++}$, it suffices to estimate

$$
J_{\ell}^{++}\left(g_{1}, g_{2}, g_{3, \ell}\right):=\int_{B_{\ell}^{++}} g_{1}\left(\xi_{1}, q_{1}\right) g_{2}\left(\xi_{2}, q_{2}\right) g_{3, \ell}\left(\xi_{1}+\xi_{2}, q_{1}+q_{2}\right) \mathrm{d} \xi_{1} \mathrm{~d} \xi_{2}\left(\mathrm{~d} q_{1}\right)_{\lambda}\left(\mathrm{d} q_{2}\right)_{\lambda}
$$

Moreover, the definition of $\Omega$ and the condition $|\Omega| \lesssim K_{3}$ give

$$
\left|\sqrt{3}\left(\xi_{1}+\xi_{2}\right)-\left(\frac{q_{1}}{\xi_{1}}-\frac{q_{2}}{\xi_{2}}\right)\right| \leqslant \frac{|\Omega|}{\left|\xi_{1} \xi_{2}\right|} \lesssim \frac{K_{3}}{M_{1} M_{2}}
$$

on $B_{\ell}^{++}$.

Now, we can define

$$
\mathcal{Q}_{1}\left(\xi_{1}, q_{1}, \xi_{2}, q_{2}\right):=\left\lfloor\frac{M_{1} M_{2}}{K_{2}}\left(q_{1}-\sqrt{3} \xi_{1}^{2}\right) / \xi_{1}\right\rfloor \in \mathbb{Z}
$$

and

$$
\mathcal{Q}_{2}\left(\xi_{1}, q_{1}, \xi_{2}, q_{2}\right):=\mathcal{Q}_{1}\left(\xi_{1}, q_{1}, \xi_{2}, q_{2}\right)-\left\lfloor\frac{M_{1} M_{2}}{K_{2}}\left(q_{2}+\sqrt{3} \xi_{2}^{2}\right) / \xi_{2}\right\rfloor \in \mathbb{Z}
$$

So we can split $B_{\ell}^{++}$according to the level sets of $\mathcal{Q}_{1}$ and $\mathcal{Q}_{2}$ :

$$
B_{\ell}^{++}=\bigsqcup_{Q_{1}, Q_{2} \in \mathbb{Z}} B_{\ell, Q_{1}, Q_{2}}
$$

where $B_{\ell, Q_{1}, Q_{2}}$ is defined as

$$
B_{\ell, Q_{1}, Q_{2}}:=\left\{\left(\xi_{1}, q_{1}\right),\left(\xi_{2}, q_{2}\right) \in B_{\ell}^{++}, \mathcal{Q}_{1}\left(\xi_{1}, q_{1}, \xi_{2}, q_{2}\right)=Q_{1}, \mathcal{Q}_{2}\left(\xi_{1}, q_{1}, \xi_{2}, q_{2}\right)=Q_{2}\right\}
$$

From definitions (5.18) and (5.19), for $\left(\xi_{1}, q_{1}\right),\left(\xi_{2}, q_{2}\right) \in B_{\ell, Q_{1}, Q_{2}}, Q_{2}$ is such that

$$
\begin{aligned}
Q_{2}=\left\lfloor\frac{M_{1} M_{2}}{K_{2}}\left(\frac{q_{1}}{\xi_{1}}-\frac{q_{2}}{\xi_{2}}-\sqrt{3}\left(\xi_{1}+\xi_{2}\right)\right)\right\rfloor & \\
& \text { or } Q_{2}=\left\lfloor\frac{M_{1} M_{2}}{K_{2}}\left(\frac{q_{1}}{\xi_{1}}-\frac{q_{2}}{\xi_{2}}-\sqrt{3}\left(\xi_{1}+\xi_{2}\right)\right)\right\rfloor+1
\end{aligned}
$$


Thus,

$$
\frac{q_{1}}{\xi_{1}}-\frac{q_{2}}{\xi_{2}}-\sqrt{3}\left(\xi_{1}+\xi_{2}\right) \in\left[\frac{K_{2}}{M_{1} M_{2}}\left(Q_{2}-1\right) ; \frac{K_{2}}{M_{1} M_{2}}\left(Q_{2}+1\right)\right]
$$

Finally, if $\left(\xi_{1}, q_{1}\right),\left(\xi_{2}, q_{2}\right) \in B_{\ell, Q_{1}, Q_{2}}$ we obtain from (5.9) and (5.20) that

$$
\begin{aligned}
\Omega\left(\xi_{1}, q_{1}, \xi_{2}, q_{2}\right)=\frac{\xi_{1} \xi_{2}}{\xi_{1}+\xi_{2}} \frac{K_{2}}{M_{1} M_{2}}\left(Q_{2}+\nu\right)\left(\left|\frac{q_{1}}{\xi_{1}}-\frac{q_{2}}{\xi_{2}}\right|+\sqrt{3}\left|\xi_{1}+\xi_{2}\right|\right) \\
=\frac{\xi_{1} \xi_{2} K_{2}}{M_{1} M_{2}}\left(Q_{2}+\nu\right)\left(2 \sqrt{3}+\frac{K_{2}}{M_{1} M_{2}} \frac{Q_{2}+\nu}{\left(\xi_{1}+\xi_{2}\right)}\right)
\end{aligned}
$$

with

$$
|\nu| \leqslant 1
$$

The choice of the parameter $\frac{K_{2}}{M_{1} M_{2}}$ in the definitions of $Q_{i}$ allows us to have $\frac{q_{1}}{\xi_{1}}$ and $\frac{q_{2}}{\xi_{2}}$ of the same order, and thus to keep an error $\nu$ of size $O(1)$ in this "change of variables". The measure of the $q_{i}$-sections of $B_{\ell, Q_{1}, Q_{2}}$ is then controled with $\frac{K_{2} M_{i}}{M_{1} M_{2}} \gtrsim 1$ (as $K_{2} \gtrsim M_{\max }$ ), $i=1,2$.

Using (5.17), we get

Moreover, by definition

$$
\left|Q_{2}\right| \lesssim \frac{K_{3}}{K_{2}}
$$

$$
\forall\left(\xi_{1}, q_{1}\right),\left(\xi_{2}, q_{2}\right) \in B_{\ell}, \ell=\left\lfloor\frac{\Omega\left(\xi_{1}, q_{1}, \xi_{2}, q_{2}\right)}{K_{2}}\right\rfloor
$$

and so a key remark is that if $\left(\xi_{1}, q_{1}\right),\left(\xi_{2}, q_{2}\right) \in B_{\ell, Q_{1}, Q_{2}}$ :

$$
\ell=\ell\left(\xi_{1}, \xi_{2}, Q_{2}\right)=\left\lfloor\frac{\xi_{1} \xi_{2}}{M_{1} M_{2}}\left(Q_{2}+\nu\right)\left(2 \sqrt{3}+\frac{K_{2}}{M_{1} M_{2}} \frac{Q_{2}+\nu}{\xi_{1}+\xi_{2}}\right)\right\rfloor
$$

Using that $\left|\xi_{i}\right| \sim M_{i}, \xi_{1}+\xi_{2} \sim M_{3},\left|Q_{2}\right| \lesssim K_{3} / K_{2}$ and that we assumed $K_{3} \leqslant 10^{-10} M_{1} M_{2} M_{3}$, we get that

$$
\left|\frac{K_{2}}{M_{1} M_{2}} \frac{Q_{2}+\nu}{\xi_{1}+\xi_{2}}\right| \leqslant 10^{-5}
$$

which means that for any fixed $Q_{1}, Q_{2}$ there is at most 10 possible values for $\ell$ such that $B_{\ell, Q, Q_{2}}$ is non empty.

Let us write $J_{\ell, Q_{1}, Q_{2}}$ the contribution of the region $B_{\ell, Q_{1}, Q_{2}}$ in the integral $J_{\ell}^{++}$. To control $J_{\ell, Q_{1}, Q_{2}}$ we first use Cauchy-Schwarz inequality in $q_{1}, q_{2}, \xi_{1}, \xi_{2}$ :

$$
\begin{aligned}
& J_{\ell, Q_{1}, Q_{2}} \lesssim\left\|g_{1}\right\|_{L^{2}\left(B_{Q_{1}}^{1}\right)}\left\|g_{2}\right\|_{L^{2}\left(B_{Q_{1}, Q_{2}}^{2}\right)} \\
& \cdot\left\{\int_{B_{\ell, Q_{1}, Q_{2}}} g_{3, \ell}^{2}\left(\xi_{1}+\xi_{2}, q_{1}+q_{2}\right) \mathrm{d} \xi_{1} \mathrm{~d} \xi_{2}\left(\mathrm{~d} q_{1}\right)_{\lambda}\left(\mathrm{d} q_{2}\right)_{\lambda}\right\}^{1 / 2}
\end{aligned}
$$

where we define

$$
\begin{aligned}
B_{Q_{1}}^{1}:=\left\{\left(\xi_{1}, q_{1}\right) \in \mathfrak{I}_{M_{1}} \times \lambda^{-1} \mathbb{Z}\right. & \\
& \left.\sqrt{3} \xi_{1}^{2}+Q_{1} \frac{K_{2}}{M_{1} M_{2}} \xi_{1} \leqslant q_{1}<\sqrt{3} \xi_{1}^{2}+\left(Q_{1}+1\right) \frac{K_{2}}{M_{1} M_{2}} \xi_{1}\right\}
\end{aligned}
$$


and

$$
\begin{aligned}
B_{Q_{1}, Q_{2}}^{2}: & =\left\{\left(\xi_{2}, q_{2}\right) \in \mathfrak{I}_{M_{2}} \times \lambda^{-1} \mathbb{Z},\right. \\
& \left.-\sqrt{3} \xi_{2}^{2}+\left(Q_{1}-Q_{2}\right) \frac{K_{2}}{M_{1} M_{2}} \xi_{2} \leqslant q_{2}<-\sqrt{3} \xi_{2}^{2}+\left(Q_{1}-Q_{2}+1\right) \frac{K_{2}}{M_{1} M_{2}} \xi_{2}\right\}
\end{aligned}
$$

Let us start by treating the integral over $B_{\ell, Q_{1}, Q_{2}}$.

If $\left(\xi_{1}, q_{1}\right),\left(\xi_{2}, q_{2}\right) \in B_{\ell, Q_{1}, Q_{2}}$, we can parametrize the $q_{i}$-sections with

$$
r_{1}:=q_{1}-\left[\sqrt{3} \xi_{1}^{2}+Q_{1} \frac{K_{2}}{M_{1} M_{2}} \xi_{1}\right]_{\lambda} \in \lambda^{-1} \mathbb{Z}
$$

and

$$
r_{2}:=q_{2}-\left[-\sqrt{3} \xi_{2}^{2}+\left(Q_{1}-Q_{2}\right) \frac{K_{2}}{M_{1} M_{2}} \xi_{2}\right]_{\lambda} \in \lambda^{-1} \mathbb{Z}
$$

such that $0 \leqslant r_{i} \lesssim \frac{K_{2} M_{i}}{M_{1} M_{2}}$.

As we assumed $M_{2} \leqslant M_{1}$, the $q_{2}$-sections of $B_{\ell, Q_{1}, Q_{2}}$ are then smaller than the $q_{1}$-sections, and thus $0 \leqslant r_{1}+r_{2} \lesssim r_{1}$. So if $\xi_{1}, \xi_{2}$ are fixed, we obtain :

$$
\begin{gathered}
\iint \mathbb{1}_{B_{\ell, Q_{1}, Q_{2}}\left(\xi_{1}, q_{1}, \xi_{2}, q_{2}\right) g_{3, \ell}^{2}\left(\xi_{1}+\xi_{2}, q_{1}+q_{2}\right)\left(\mathrm{d} q_{1}\right)_{\lambda}\left(\mathrm{d} q_{2}\right)_{\lambda}} \\
=\iint \mathbb{1}_{\left[0 ; K_{2} / M_{2}\right]}\left(r_{1}\right) \mathbb{1}_{\left[0 ; K_{2} / M_{1}\right]}\left(r_{2}\right) g_{3, \ell}^{2}\left(\xi_{1}+\xi_{2},\right. \\
\left.\left[\sqrt{3} \xi_{1}^{2}+Q_{1} \frac{K_{2}}{M_{1} M_{2}} \xi_{1}\right]_{\lambda}+r_{1}+\left[-\sqrt{3} \xi_{2}^{2}+\left(Q_{1}-Q_{2}\right) \frac{K_{2}}{M_{1} M_{2}} \xi_{2}\right]_{\lambda}+r_{2}\right)\left(\mathrm{d} r_{1}\right)_{\lambda}\left(\mathrm{d} r_{2}\right)_{\lambda} \\
\lesssim \frac{K_{2}}{M_{1}} \int \mathbb{1}_{\left[0 ; K_{2} / M_{2}\right]}(|r|) g_{3, \ell}^{2}\left(\xi_{1}+\xi_{2},\right. \\
\left.\left[\sqrt{3} \xi_{1}^{2}+Q_{1} \frac{K_{2}}{M_{1} M_{2}} \xi_{1}-\sqrt{3} \xi_{2}^{2}+\left(Q_{1}-Q_{2}\right) \frac{K_{2}}{M_{1} M_{2}} \xi_{2}\right]_{\lambda}+r\right)(\mathrm{d} r)_{\lambda}
\end{gathered}
$$

The integral over $B_{\ell, Q_{1}, Q_{2}}$ is thus controled by

$$
\begin{aligned}
J_{\ell, Q_{1}, Q_{2}} \lesssim & \left.\left(\frac{K_{2}}{M_{1}}\right)^{1 / 2}\left\|g_{1}\right\|_{L^{2}\left(B_{Q_{1}}^{1}\right)}\left\|g_{2}\right\|_{L^{2}\left(B_{Q_{1}, Q_{2}}^{2}\right.}\right)\left\{\int _ { \mathbb { R } ^ { 2 } } \int \mathbb { 1 } _ { [ 0 ; K _ { 2 } / M _ { 2 } } ( | r | ) g _ { 3 , \ell } ^ { 2 } \left(\xi_{1}+\xi_{2},\right.\right. \\
& {\left.\left.\left[\sqrt{3} \xi_{1}^{2}+Q_{1} \frac{K_{2}}{M_{1} M_{2}} \xi_{1}-\sqrt{3} \xi_{2}^{2}+\left(Q_{1}-Q_{2}\right) \frac{K_{2}}{M_{1} M_{2}} \xi_{2}\right]_{\lambda}+r\right) \mathrm{~d} \xi_{1} \mathrm{~d} \xi_{2}(\mathrm{~d} r)_{\lambda}\right\}^{1 / 2} }
\end{aligned}
$$

It remains to sum those contributions : using the previous estimate,

$$
\begin{gathered}
J=\sum_{|\ell| \lesssim K_{3} / K_{2}} \sum_{Q_{1} \in \mathbb{Z}\left|Q_{2}\right| \lesssim K_{3} / K_{2}} J_{\ell, Q_{1}, Q_{2}} \\
\lesssim \sum_{Q_{1} \in \mathbb{Z}\left|Q_{2}\right| \lesssim K_{3} / K_{2}} \sum\left(\frac{K_{2}}{M_{1}}\right)^{1 / 2}\left\|g_{1}\right\|_{L^{2}\left(B_{Q_{1}}^{1}\right)}\left\|g_{2}\right\|_{L^{2}\left(B_{Q_{1}, Q_{2}}^{2}\right)} \\
\cdot\left\{\sum _ { | \ell | \lesssim K _ { 3 } / K _ { 2 } } \int _ { \mathbb { R } ^ { 2 } } \int \mathbb { 1 } _ { [ 0 ; K _ { 2 } / M _ { 2 } ] } ( | r | ) g _ { 3 , \ell } ^ { 2 } \left(\xi_{1}+\xi_{2},\right.\right. \\
\left.\left.\left[\sqrt{3} \xi_{1}^{2}+Q_{1} \frac{K_{2}}{M_{1} M_{2}} \xi_{1}-\sqrt{3} \xi_{2}^{2}+\left(Q_{1}-Q_{2}\right) \frac{K_{2}}{M_{1} M_{2}} \xi_{2}\right]_{\lambda}+r\right) \mathrm{~d} \xi_{1} \mathrm{~d} \xi_{2}(\mathrm{~d} r)_{\lambda}\right\}^{1 / 2}
\end{gathered}
$$


Next, a use of Cauchy-Schwarz inequality in $Q_{2}$ then $Q_{1}$ gives

$$
\begin{aligned}
J \lesssim\left(\frac{K_{2}}{M_{1}}\right)^{1 / 2}\left(\sum_{Q_{1} \in \mathbb{Z}}\left\|g_{1}\right\|_{L^{2}\left(B_{Q_{1}}^{1}\right)}^{2}\right)^{1 / 2}\left(\sum_{Q_{1} \in \mathbb{Z}\left|Q_{2}\right| \lesssim K_{3} / K_{2}} \sum_{\left.g_{2} \|_{L^{2}\left(B_{Q_{1}, Q_{2}}^{2}\right)}^{2}\right)^{1 / 2}}\right. \\
\cdot\left\{\operatorname { s u p } _ { Q _ { 1 } } \sum _ { | Q _ { 2 } | \lesssim K _ { 3 } / K _ { 2 } } \sum _ { | \ell | \lesssim K _ { 3 } / K _ { 2 } } \int _ { \mathbb { R } ^ { 2 } } \int \mathbb { 1 } _ { [ 0 ; K _ { 2 } / M _ { 2 } ] } ( | r | ) g _ { 3 , \ell } ^ { 2 } \left(\xi_{1}+\xi_{2},\right.\right. \\
{\left.\left.\left[\sqrt{3} \xi_{1}^{2}+Q_{1} \frac{K_{2}}{M_{1} M_{2}} \xi_{1}-\sqrt{3} \xi_{2}^{2}+\left(Q_{1}-Q_{2}\right) \frac{K_{2}}{M_{1} M_{2}} \xi_{2}\right]_{\lambda}+r\right) \mathrm{~d} \xi_{1} \mathrm{~d} \xi_{2}(\mathrm{~d} r)_{\lambda}\right\}^{1 / 2} }
\end{aligned}
$$

Now, from the definitions of $B_{Q_{1}}^{1}(5.23)$ and $B_{Q_{1}, Q_{2}}^{2}(5.24)$ :

$$
\left(\sum_{Q_{1} \in \mathbb{Z}}\left\|g_{1}\right\|_{L^{2}\left(B_{Q_{1}}^{1}\right)}^{2}\right)^{1 / 2}=\left\|g_{1}\right\|_{L_{\xi_{1}, q_{1}}^{2}}=\left\|f_{1}\right\|_{L^{2}}
$$

and

$$
\begin{aligned}
& \left(\sum_{Q_{1} \in \mathbb{Z}} \sum_{\left|Q_{2}\right| \lesssim K_{3} / K_{2}}\left\|g_{2}\right\|_{L^{2}\left(B_{Q_{1}, Q_{2}}^{2}\right)}^{2}\right)^{1 / 2} \lesssim\left(\frac{K_{3}}{K_{2}}\right)^{1 / 2}\left(\sup _{Q_{2}} \sum_{Q_{1} \in \mathbb{Z}}\left\|g_{2}\right\|_{L^{2}\left(B_{Q_{1}, Q_{2}}\right)}^{2}\right)^{1 / 2} \\
& =\left(\frac{K_{3}}{K_{2}}\right)^{1 / 2}\left\|g_{2}\right\|_{L_{\xi_{2}, q_{2}}^{2}}=\left(\frac{K_{3}}{K_{2}}\right)^{1 / 2}\left\|f_{2}\right\|_{L^{2}}
\end{aligned}
$$

To conclude, it suffices to prove

$$
\begin{aligned}
\sup _{Q_{1}} & \sum_{\left|Q_{2}\right| \lesssim K_{3} / K_{2}} \sum_{|\ell| \lesssim K_{3} / K_{2}} \int_{\mathbb{R}^{2}} \int \mathbb{1}_{\left[0 ; K_{2} / M_{2}\right]}(|r|) g_{3, \ell}^{2}\left(\xi_{1}+\xi_{2},\right. \\
& {\left.\left[\sqrt{3} \xi_{1}^{2}+Q_{1} \frac{K_{2}}{M_{1} M_{2}} \xi_{1}-\sqrt{3} \xi_{2}^{2}+\left(Q_{1}-Q_{2}\right) \frac{K_{2}}{M_{1} M_{2}} \xi_{2}\right]_{\lambda}+r\right) \mathrm{d} \xi_{1} \mathrm{~d} \xi_{2}(\mathrm{~d} r)_{\lambda} } \\
& \lesssim \frac{K_{2}}{M_{2} M_{3}}\left\|f_{3}\right\|_{L^{2}}
\end{aligned}
$$

Here, we can see the interest of splitting $f_{3}^{\#}$ over $\ell$ : the sum over $\ell$ is controled by the sum over $Q_{2}$ thanks to (5.22), whereas a direct estimate on this sum would lose an additional factor $K_{3} / K_{2}$ (or in other words, when $\xi_{1}, \xi_{2}, Q_{2}$ are fixed, we do not have the contribution of the full $L^{2}$ norm of $f_{3}^{\#}$ in the $\theta$ variable, which allows us to sum those contributions without loosing an additionnal factor).

We begin the proof of (5.25) with the change of variables $\xi_{1} \mapsto \xi:=\xi_{1}+\xi_{2}$ : the left-hand side now reads

$$
\begin{aligned}
\sup _{Q_{1}} \sum_{\left|Q_{2}\right| \lesssim K_{3} / K_{2}} \sum_{|\ell| \lesssim K_{3} / K_{2}} & \int_{\mathbb{R}^{2}} \int \mathbb{1}_{\left[0 ; K_{2} / M_{2}\right]}(|r|) g_{3, \ell}^{2}(\xi \\
& {\left.\left[\sqrt{3} \xi\left(\xi-2 \xi_{2}\right)+Q_{1} \frac{K_{2}}{M_{1} M_{2}} \xi-Q_{2} \frac{K_{2}}{M_{1} M_{2}} \xi_{2}\right]_{\lambda}+r\right) \mathrm{d} \xi_{2} \mathrm{~d} \xi(\mathrm{d} r)_{\lambda} }
\end{aligned}
$$


Now, using (5.22) and the definition of $g_{3, \ell}$, we have that for fixed $\xi, Q_{1}, \xi_{2}, Q_{2}, r$ :

$$
\begin{aligned}
\sum_{|\ell| \lesssim K_{3} / K_{2}} g_{3, \ell}^{2}(\xi,[ & \left.\left.\sqrt{3} \xi\left(\xi-2 \xi_{2}\right)+Q_{1} \frac{K_{2}}{M_{1} M_{2}} \xi-Q_{2} \frac{K_{2}}{M_{1} M_{2}} \xi_{2}\right]_{\lambda}+r\right) \\
\lesssim \int_{\mathbb{R}} \mathbb{1} & \left(\theta \in \left[\frac{\left(\xi-\xi_{2}\right) \xi_{2} K_{2}}{M_{1} M_{2}}\left(Q_{2}-2\right)\left(2 \sqrt{3}+\frac{K_{2}}{M_{1} M_{2}} \frac{Q_{2}-2}{\xi}\right) ;\right.\right. \\
& \left.\left.\frac{\left(\xi-\xi_{2}\right) \xi_{2} K_{2}}{M_{1} M_{2}}\left(Q_{2}+2\right)\left(2 \sqrt{3}+\frac{K_{2}}{M_{1} M_{2}} \frac{Q_{2}+2}{\xi}\right)\right]\right) \\
& \cdot\left(f_{3}^{\#}\right)^{2}\left(\theta, \xi,\left[\sqrt{3} \xi\left(\xi-2 \xi_{2}\right)+Q_{1} \frac{K_{2}}{M_{1} M_{2}} \xi-Q_{2} \frac{K_{2}}{M_{1} M_{2}} \xi_{2}\right]_{\lambda}+r\right) \mathrm{d} \theta
\end{aligned}
$$

Now, fixing only $\xi$, and $Q_{1}$, integrating in $\xi_{2}$ and $r$ and summing over $Q_{2}$, we can write the previous term as

$$
\begin{aligned}
\sum_{\left|Q_{2}\right| \lesssim K_{3} / K_{2}} \int_{\mathfrak{J}_{M_{2}}} \int \mathbb{1}_{\left[0 ; K_{2} / M_{2}\right]}(|r|) \int_{\mathbb{R}} \mathbb{1}\left\{\theta \in I\left(\xi, \xi_{2}, Q_{2}\right)\right\} \\
\cdot\left(f_{3}^{\#}\right)^{2}\left(\theta, \xi,\left[\varphi\left(\xi, Q_{1}, \xi_{2}, Q_{2}\right)\right]_{\lambda}+r\right) \mathrm{d} \theta(\mathrm{d} r)_{\lambda} \mathrm{d} \xi_{2}
\end{aligned}
$$

where the interval $I\left(\xi, \xi_{2}, Q_{2}\right)$ is defined as

$$
\begin{aligned}
I\left(\xi, \xi_{2}, Q_{2}\right):=\left[\frac{\left(\xi-\xi_{2}\right) \xi_{2} K_{2}}{M_{1} M_{2}}\left(Q_{2}-2\right)\right. & \left(2 \sqrt{3}+\frac{K_{2}}{M_{1} M_{2}} \frac{Q_{2}-2}{\xi}\right) ; \\
& \left.\frac{\left(\xi-\xi_{2}\right) \xi_{2} K_{2}}{M_{1} M_{2}}\left(Q_{2}+2\right)\left(2 \sqrt{3}+\frac{K_{2}}{M_{1} M_{2}} \frac{Q_{2}+2}{\xi}\right)\right]
\end{aligned}
$$

and the function $\varphi$ is defined as

$$
\varphi\left(\xi, Q_{1}, \xi_{2}, Q_{2}\right):=\sqrt{3} \xi\left(\xi-2 \xi_{2}\right)+Q_{1} \frac{K_{2}}{M_{1} M_{2}} \xi-Q_{2} \frac{K_{2}}{M_{1} M_{2}} \xi_{2}
$$

In order to recover the $L^{2}$ norm of $f_{3}^{\#}$ in $q$, we decompose the previous term in

$$
\begin{aligned}
\lambda \int_{\lambda^{-1} \mathbb{Z}} \sum_{\left|Q_{2}\right| \lesssim K_{3} / K_{2}} \iint_{\Lambda_{n}\left(\xi, Q_{1}, Q_{2}\right)} \int_{\mathbb{R}} \mathbb{1}\left\{\theta \in I\left(\xi, \xi_{2}, Q_{2}\right)\right\} \\
\cdot\left(f_{3}^{\#}\right)^{2}(\theta, \xi, n) \mathrm{d} \theta\left(\mathrm{d} \xi_{2}(\mathrm{~d} r)_{\lambda}\right)(\mathrm{d} n)_{\lambda}
\end{aligned}
$$

where the set $\Lambda_{n}\left(\xi, Q_{1}, Q_{2}\right) \subset \mathbb{R} \times \lambda^{-1} \mathbb{Z}$ for $n \in \lambda^{-1} \mathbb{Z}$ is defined as

$$
\begin{aligned}
& \Lambda_{n}\left(\xi, Q_{1}, Q_{2}\right):=\left\{\left(\xi_{2}, r\right) \in \mathfrak{I}_{M_{2}} \times\left[-\frac{K_{2}}{M_{2}} ; \frac{K_{2}}{M_{2}}\right],\right. \\
& \varphi\left(\xi, Q_{1}, \xi_{2}, Q_{2}\right) \in\left[n-r ; n+\lambda^{-1}-r[\}\right.
\end{aligned}
$$

First, using the localizations $|\xi| \sim M_{3},\left|\xi_{2}\right| \sim M_{2}$ and $\left|\xi-\xi_{2}\right| \sim M_{1}$ and the conditions $\left|Q_{2}\right| \lesssim K_{3} / K_{2}$ and $K_{3} \leqslant 10^{-10} M_{1} M_{2} M_{3}$, we have for any $\xi, \xi_{2}, Q_{2}$ :

$$
I\left(\xi, \xi_{2}, Q_{2}\right) \subset\left\{|\theta| \in\left[c^{-1} K_{2}\left(Q_{2}-2\right), c K_{2}\left(Q_{2}+2\right)\right]\right\}
$$

for an absolute constant $c>0$. 
Thus we are left with estimating

$$
\begin{aligned}
\lambda \int_{\lambda^{-1} \mathbb{Z}} \sum_{\left|Q_{2}\right| \lesssim K_{3} / K_{2}} \int_{\mathbb{R}} \mathbb{1}\left\{|\theta| \in\left[c^{-1} K_{2}\left(Q_{2}-2\right) ; c K_{2}\left(Q_{2}+2\right)\right]\right\} \\
\cdot\left|\Lambda_{n}\left(\xi, Q_{1}, Q_{2}\right)\right|\left(f_{3}^{\#}\right)^{2}(\theta, \xi, n) \mathrm{d} \theta(\mathrm{d} n)_{\lambda}
\end{aligned}
$$

We trivially control the measure of the $r$-sections of $\Lambda_{n}$ with $2 \frac{K_{2}}{M_{2}}$. It remains to estimate the measure of the projection of $\Lambda_{n}$ on the $\xi_{2}$ axis, uniformly in $n, \xi, Q_{1}$ and $Q_{2}$. To do so, we are going to make a good use of lemma 5.3. We are then left to compute $\frac{\partial \varphi}{\partial \xi_{2}}$ :

$$
\frac{\partial \varphi}{\partial \xi_{2}}=-2 \sqrt{3} \xi-Q_{2} \frac{K_{2}}{M_{1} M_{2}}
$$

Now, as $\left|Q_{2}\right| \lesssim K_{3} / K_{2}$ and $K_{3} \leqslant 10^{-10} M_{1} M_{2} M_{3}$, we obtain that

$$
\left|\frac{\partial \varphi}{\partial \xi_{2}}\right| \sim 2 \sqrt{3}|\xi| \sim M_{3}
$$

So, applying (5.3), we get that the projection of $\Lambda_{n}\left(\xi, Q_{1}, Q_{2}\right)$ on the $\xi_{2}$ axis is controled by $\lambda^{-1} M_{3}^{-1}$. A use of lemma 5.2 finally leads to

$$
\left|\Lambda_{n}\left(\xi, Q_{1}, Q_{2}\right)\right| \lesssim \lambda^{-1} \frac{K_{2}}{M_{2} M_{3}}
$$

uniformly in $n, \xi, Q_{1}, Q_{2}$.

Getting back to (5.26), we have

$$
\begin{aligned}
(5.26) \lesssim \frac{K_{2}}{M_{2} M_{3}} \int_{\lambda^{-1} \mathbb{Z}} \int_{\mathbb{R}}\left(\sum_{\left|Q_{2}\right| \lesssim K_{3} / K_{2}} \mathbb{1}\left\{\theta \in\left[c^{-1} K_{2}\left(Q_{2}-2\right) ; c K_{2}\left(Q_{2}+2\right)\right]\right\}\right) \\
\cdot\left(f_{3}^{\#}\right)^{2}(\theta, \xi, n) \mathrm{d} \theta(\mathrm{d} n)_{\lambda} \\
\lesssim \frac{K_{2}}{M_{2} M_{3}} \int_{\lambda^{-1} \mathbb{Z}} \int_{\mathbb{R}} \mathbb{1}\left\{|\theta| \in I_{\leqslant}\right\}\left(f_{3}^{\#}\right)^{2}(\theta, \xi, n) \mathrm{d} \theta(\mathrm{d} n)_{\lambda}
\end{aligned}
$$

Now, neglecting the $\theta$ localization and integrating in $\xi$, we finally get (5.25), which completes the proof of the proposition.

Remark 5.8. In the case $(x, y) \in \mathbb{T}^{2}([25$, Lemma 3.1$])$, we can still use lemma 5.3 , but since $\xi_{2} \in \mathbb{Z}$ in that case, we have to use (5.4) instead of (5.3), and thus we have the rougher estimate

$$
\left|\Lambda_{n}\right| \lesssim \frac{K_{2}}{M_{2}}\left(1+M_{3}^{-1}\right) \lesssim \frac{K_{2}}{M_{2}}
$$

as $M_{i} \geqslant 1$ for localized functions on $\mathbb{T}^{2}$. This is the main obstacle to recover the same estimate as in $\mathbb{R}^{2}$ or $\mathbb{R} \times \mathbb{T}$, and the cause of the logarithmic divergence in the energy estimate.

The following corollary summarizes the estimates on $\int f_{1} \star f_{2} \cdot f_{3}$ according to the relations between the $M$ 's and the $K$ 's : 


\section{Corollary 5.9}

Let $f_{i} \in L^{2}\left(\mathbb{R}^{2} \times \lambda^{-1} \mathbb{Z}\right)$ be positive functions with the support condition $\operatorname{supp} f_{i} \subset$ $D_{\lambda, M_{i}, \leqslant K_{i}}, i=1,2,3$. We assume $K_{\text {med }} \geqslant M_{\max } \geqslant 1$.

(a) If $K_{\max } \leqslant 10^{-10} M_{1} M_{2} M_{3}$ then

$$
\int_{\mathbb{R}^{2} \times \lambda^{-1} \mathbb{Z}} f_{1} \star f_{2} \cdot f_{3} \lesssim\left(M_{\min } \wedge M_{\min }^{-1}\right)^{1 / 2} M_{\max }^{-1} \prod_{i=1}^{3} K_{i}^{1 / 2}\left\|f_{i}\right\|_{L^{2}}
$$

(b) If $K_{\max } \gtrsim M_{1} M_{2} M_{3}$ and $\left(M_{i}, K_{i}\right)=\left(M_{\min }, K_{\max }\right)$ for an $i \in\{1,2,3\}$ then

$$
\int_{\mathbb{R}^{2} \times \lambda^{-1} \mathbb{Z}} f_{1} \star f_{2} \cdot f_{3} \lesssim\left(1 \wedge M_{\min }\right)^{1 / 4} M_{\max }^{-1} \prod_{i=1}^{3} K_{i}^{1 / 2}\left\|f_{i}\right\|_{L^{2}}
$$

(c) If $K_{\max } \gtrsim M_{1} M_{2} M_{3}$ but $\left(M_{i}, K_{i}\right) \neq\left(M_{\min }, K_{\max }\right)$ for any $i=1,2,3$ then

$$
\int_{\mathbb{R}^{2} \times \lambda^{-1} \mathbb{Z}} f_{1} \star f_{2} \cdot f_{3} \lesssim\left(1 \vee M_{\min }\right)^{1 / 4} M_{\max }^{-5 / 4} \prod_{i=1}^{3} K_{i}^{1 / 2}\left\|f_{i}\right\|_{L^{2}}
$$

Proof :

Using the symmetry property (5.11), we can assume $K_{3}=K_{\max }$. Note that, since $M_{\max } \geqslant 1$ and in order for the integral to be non zero, we must have $\left(1 \vee M_{\text {min }}\right) \lesssim M_{\text {med }} \sim M_{\text {max }}$. Then we treat the different cases.

Case (a) : This has already been proven in the previous proposition in the case $M_{\min } \geqslant 1$. If $M_{\min } \leqslant 1,(5.27)$ follows from (5.8), since $K_{3}=K_{\max } \geqslant\left(K_{1} \vee K_{2}\right) \geqslant M_{\max }$.

Case (b) : $M_{3}=M_{m i n}$. Then, if $M_{3} \geqslant 1$, (5.28) follows from (5.7) since

$$
\left\langle\left(K_{1} \vee K_{2}\right)^{1 / 4}\left(M_{1} \wedge M_{2}\right)^{1 / 4}\right\rangle \lesssim\left(K_{1} \vee K_{2}\right)^{1 / 2}
$$

as $\left(K_{1} \vee K_{2}\right) \gtrsim M_{\max }$.

If $M_{3} \leqslant 1$, since this is symmetrical in $f_{1}$ and $f_{2}$ we may assume that $K_{1}=K_{1} \wedge K_{2}$. Then we apply (5.7) with $f_{1}$ and $f_{3}$ to get (5.28) since $K_{3}^{-1 / 4} \lesssim M_{\min }^{-1 / 4} M_{\max }^{-1 / 2}$ and $K_{2}^{-1 / 2}=$ $K_{\text {med }}^{-1 / 2} \lesssim M_{\text {max }}^{-1 / 2}$.

Case (c) : Again, (5.29) follows from (5.7) since

$$
\left\langle\left(K_{1} \vee K_{2}\right)^{1 / 4}\left(M_{1} \wedge M_{2}\right)^{1 / 4}\right\rangle \lesssim\left(K_{1} \vee K_{2}\right)^{1 / 2} M_{\text {max }}^{-1 / 4}\left(1 \vee M_{\text {min }}\right)^{1 / 4}
$$

We conclude this section by stating another estimate which takes into account the weight in the definition of the energy space : 


\section{Proposition 5.10}

Let $f_{i} \in L^{2}\left(\mathbb{R}^{2} \times \lambda^{-1} \mathbb{Z}\right)$ be positive functions with the support condition $\operatorname{supp} f_{i} \subset$ $D_{\lambda, M_{i}, K_{i}}, i=1,2$ for $M_{3}>0, K_{3} \geqslant 1$. Then

$$
\left\|\mathbb{1}_{D_{\lambda, M_{3}, K_{3}}} \cdot f_{1} \star f_{2}\right\|_{L^{2}} \lesssim\left(1 \vee M_{1}\right) M_{\min }^{1 / 2} K_{\min }^{1 / 2}\left\|p \cdot f_{1}\right\|_{L^{2}}\left\|f_{2}\right\|_{L^{2}}
$$

\section{Proof :}

We follow $[9$, Corollaire $5.3(\mathrm{~b}) \&(\mathrm{c})]$ : we split the cases $M_{1} \lesssim 1$ or $M_{1} \gtrsim 1$ and we decompose $f_{1}$ on its $y$ frequency in order to estimate $p(\xi, q) \sim 1+\frac{|q|}{|\xi|\langle\xi\rangle}$.

Case 1 :If $M_{1} \geqslant 1$.

We then have $p(\xi, q) \sim 1+\frac{|q|}{|\xi|^{2}}$. We split

$$
f_{1}=\sum_{L \geqslant M_{1}^{2}} f_{1}^{L}=\mathbb{1}_{I_{\leqslant M_{1}^{2}}}(q) f_{1}+\sum_{L>M_{1}^{2}} \mathbb{1}_{I_{L}}(q) f_{1}
$$

such that

$$
\left\|\mathbb{1}_{D_{\lambda, M_{3}, K_{3}}} \cdot f_{1} \star f_{2}\right\|_{L^{2}} \lesssim \sum_{L \geqslant M_{1}^{2}} L^{1 / 2} M_{m i n}^{1 / 2} K_{m i n}^{1 / 2}\left\|f_{1}^{L}\right\|_{L^{2}}\left\|f_{2}\right\|_{L^{2}}
$$

after using (5.1). Now, for $L=M_{1}^{2}$ we have $L^{-1 / 2} p \sim M_{1}^{-1}\left(1+M_{1}^{-2}|q|\right) \gtrsim M_{1}^{-1}=L^{1 / 2} M_{1}^{-2}$, and for $L>M_{1}^{2}$ we also have $L^{-1 / 2} p \sim L^{-1 / 2}\left(1+L M_{1}^{-2}\right) \gtrsim L^{1 / 2} M_{1}^{-2}$. Thus, using CauchySchwarz inequality in $L$, we obtain

$$
\begin{aligned}
&\left\|\mathbb{1}_{D_{\lambda, M_{3}, K_{3}}} \cdot f_{1} \star f_{2}\right\|_{L^{2}} \lesssim M_{\text {min }}^{1 / 2} K_{\text {min }}^{1 / 2}\left\|f_{2}\right\|_{L^{2}} \sum_{L \geqslant M_{1}^{2}} L^{-1 / 2} M_{1}^{2}\left\|p \cdot f_{1}^{L}\right\|_{L^{2}} \\
& \lesssim M_{1}^{2} M_{\text {min }}^{1 / 2} K_{\text {min }}^{1 / 2} \cdot M_{1}^{-1}\left\|p \cdot f_{1}\right\|_{L^{2}}
\end{aligned}
$$

Case 2 : If $M_{1} \leqslant 1$.

This time, we split the $y$ frequency for $L \geqslant 1$ since for $M_{1}<\lambda^{-1}$ there is just the frequency $q=0$ :

$$
f_{1}=\sum_{L \geqslant 1} f_{1}^{L}=\mathbb{1}_{I_{\leqslant 1}}(q) f_{1}+\sum_{L>1} \mathbb{1}_{I_{L}}(q) f_{1}
$$

For $L=1$, we have $L^{-1 / 2} p \gtrsim 1=L^{1 / 2}$, and for $L>1$, we also have $L^{-1 / 2} p \gtrsim L^{1 / 2} M_{1}^{-1} \gtrsim$ $L^{1 / 2}$. Thus, using again (5.1) and then Cauchy-Schwarz inequality in $L$, we only get in that case

$$
\begin{aligned}
\left\|\mathbb{1}_{D_{\lambda, M_{3}, K_{3}}} \cdot f_{1} \star f_{2}\right\|_{L^{2}} & \lesssim \sum_{L \geqslant 1} L^{1 / 2} M_{\min }^{1 / 2} K_{\min }^{1 / 2}\left\|f_{1}^{L}\right\|_{L^{2}}\left\|f_{2}\right\|_{L^{2}} \\
& \lesssim M_{\min }^{1 / 2} K_{\min }^{1 / 2} \sum_{L \geqslant 1} L^{-1 / 2}\left\|p \cdot f_{1}^{L}\right\|_{L^{2}}\left\|f_{2}\right\|_{L^{2}} \\
& \left.\quad \lesssim M_{m i n}^{1 / 2} K_{\text {min }}^{1 / 2}\left\|p \cdot f_{1}\right\|\right|_{L^{2}}\left\|f_{2}\right\|_{L^{2}}
\end{aligned}
$$




\section{Bilinear estimates}

The aim of this section is to prove (1.12) and (1.15). We will treat separately the interactions Low $\times$ High $\rightarrow$ High, High $\times$ High $\rightarrow$ Low and Low $\times$ Low $\rightarrow$ Low. Those are the only possible interactions, since for functions $f_{i}$ localized in $\left|\xi_{i}\right| \sim M_{i}$, we have

$$
\int f_{1} \star f_{2} \cdot f_{3} \neq 0 \Rightarrow M_{\text {min }} \lesssim M_{\text {med }} \sim M_{\max }
$$

\subsection{For the equation}

We first prove (1.12).

\section{Lemma 6.1 (Low $\times$ High $\rightarrow$ High $)$}

Let $M_{1}, M_{2}, M_{3} \in 2^{\mathbb{Z}}$ with $\left(1 \vee M_{1}\right) \lesssim M_{2} \sim M_{3}$ and $b_{1} \in\left[0 ; 1 / 2\left[\right.\right.$. Then for $u_{M_{1}} \in N_{\lambda, M_{1}}^{0}$ and $v_{M_{2}} \in N_{\lambda, M_{2}}^{0}$, we have

$$
\left\|\left.P_{M_{3}} \partial_{x}\left(u_{M_{1}} \cdot v_{M_{2}}\right)\right|_{N_{\lambda, M_{3}}^{b_{1}}} \lesssim M_{1}^{1 / 2}\right\| u_{M_{1}}\left\|_{F_{\lambda, M_{1}}^{0}}\right\| v_{M_{2}} \|_{F_{\lambda, M_{2}}^{0}}
$$

\section{Proof :}

By definition, the left-hand side of (6.1) is

$$
\sup _{t_{M_{3}} \in \mathbb{R}}\left\|\left(\tau-\omega+i M_{3}\right)^{-1} p \cdot \mathcal{F}\left\{\chi_{M_{3}^{-1}}\left(t-t_{M_{3}}\right) P_{M_{3}} \partial_{x}\left(u_{M_{1}} \cdot v_{M_{2}}\right)\right\}\right\|_{X_{\lambda, M_{3}}^{b_{1}}}
$$

Let $\gamma: \mathbb{R} \rightarrow[0 ; 1]$ be a smooth partition of unity, satisfying supp $\gamma \subset[-1 ; 1]$ and

$$
\forall x \in \mathbb{R}, \sum_{m \in \mathbb{Z}} \gamma(x-m)=1
$$

Since $\left(1 \vee M_{1}\right) \lesssim M_{2} \sim M_{3}$, we have

$$
\begin{aligned}
\chi_{M_{3}^{-1}}\left(t-t_{M_{3}}\right)= & \sum_{|m|,|n| \leqslant 100} \chi_{M_{3}^{-1}}\left(t-t_{M_{3}}\right) \gamma_{M_{2}^{-1}}\left(t-t_{M_{3}}-M_{2}^{-1} m\right) \\
& \cdot \gamma_{\left(1 \vee M_{1}\right)^{-1}}\left(t-t_{M_{3}}-M_{2}^{-1} m-\left(1 \vee M_{1}\right)^{-1} n\right)
\end{aligned}
$$

Since we take the supremum in $m$ and $n$, without loss of generality, we can assume $m=$ $n=0$. Thus, if we define

$$
\begin{aligned}
f_{1}^{\left(1 \vee M_{1}\right)}:=\chi_{\left(1 \vee M_{1}\right)}(\tau-\omega) \mathcal{F}\left(\gamma_{\left(1 \vee M_{1}\right)^{-1}}\left(t-t_{M_{3}}\right) u_{M_{1}}\right) \text { and } \\
f_{1}^{K_{1}}:=\rho_{K_{1}}(\tau-\omega) \mathcal{F}\left(\gamma_{\left(1 \vee M_{1}\right)^{-1}}\left(t-t_{M_{3}}\right) u_{M_{1}}\right), \text { if } K_{1}>\left(1 \vee M_{1}\right)
\end{aligned}
$$

and as well for $v$

$$
\begin{aligned}
& f_{2}^{M_{2}}:=\chi_{M_{2}}(\tau-\omega) \mathcal{F}\left(\gamma_{M_{2}^{-1}}\left(t-t_{M_{3}}\right) v_{M_{2}}\right) \text { and } \\
& f_{2}^{K_{2}}:=\rho_{K_{2}}(\tau-\omega) \mathcal{F}\left(\gamma_{M_{2}^{-1}}\left(t-t_{M_{3}}\right) v_{M_{2}}\right), \text { if } K_{2}>M_{2}
\end{aligned}
$$


by splitting the term in the left-hand side according to its modulations, we then get

$$
\begin{gathered}
\left\|P_{M_{3}} \partial_{x}\left(u_{M_{1}} \cdot v_{M_{2}}\right)\right\|_{N_{\lambda, M_{3}}^{b_{1}}} \\
\lesssim \sup _{t_{M_{3}} \in \mathbb{R}} \sum_{K_{1} \geqslant\left(1 \vee M_{1}\right)} \sum_{K_{2} \geqslant M_{2}}\left\|\left(\tau-\omega+i M_{3}\right)^{-1} p \cdot \mathcal{F}\left\{P_{M_{3}} \partial_{x} \mathcal{F}^{-1}\left(f_{1}^{K_{1}} \star f_{2}^{K_{2}}\right)\right\}\right\|_{X_{\lambda, M_{3}}} \\
=\sup _{t_{M_{3}} \in \mathbb{R}} \sum_{K_{1} \geqslant\left(1 \vee M_{1}\right)} \sum_{K_{2} \geqslant M_{2}} \sum_{K_{3} \geqslant 1} K_{3}^{1 / 2} \beta_{M_{3}, K_{3}}^{b_{1}} \\
\cdot \|\left(\tau-\omega+i M_{3}\right)^{-1} p \cdot \rho_{K_{3}}(\tau-\omega) \mathcal{F}\left\{P_{\left.M_{3} \partial_{x} \mathcal{F}^{-1}\left(f_{1}^{K_{1}} \star f_{2}^{K_{2}}\right)\right\} \|_{L^{2}}}\right.
\end{gathered}
$$

Let us start with the modulations $K_{3}<M_{3}$ : the first factor in the previous norm allows us to gain a factor $\left(M_{3} \vee K_{3}\right)^{-1}$ which makes up for the derivative, thus

$$
\begin{aligned}
\sum_{1 \leqslant K_{3}<M_{3}} K_{3}^{1 / 2}\left\|\left(\tau-\omega+i M_{3}\right)^{-1} p \cdot \rho_{K_{3}}(\tau-\omega) \mathcal{F}\left\{P_{M_{3}} \partial_{x} \mathcal{F}^{-1}\left(f_{1}^{K_{1}} \star f_{2}^{K_{2}}\right)\right\}\right\|_{L^{2}} \\
\\
\lesssim \sum_{1 \leqslant K_{3}<M_{3}} K_{3}^{1 / 2} \| \mathbb{1}_{D_{\lambda, M_{3}, \leqslant M_{3}} \cdot p \cdot f_{1}^{K_{1}} \star f_{2}^{K_{2}} \|_{L^{2}}}
\end{aligned}
$$

and using that $\sum_{1 \leqslant K_{3}<M_{3}} K_{3}^{1 / 2} \lesssim M_{3}^{1 / 2}$ we get that the previous sum is controlled with

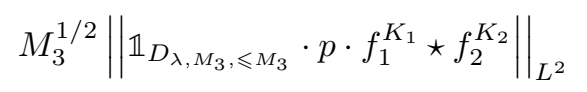

Proceeding as well for the modulations $K_{3} \geqslant M_{3}$ and choosing a factor $K_{3}^{-1}$ instead of $M_{3}^{-1}$, we get now

$$
\begin{gathered}
\sum_{K_{3} \geqslant M_{3}} K_{3}^{1 / 2} \beta_{M_{3}, K_{3}}^{b_{1}}\left\|\left(\tau-\omega+i M_{3}\right)^{-1} p \cdot \rho_{K_{3}}(\tau-\omega) \mathcal{F}\left\{P_{M_{3}} \partial_{x} \mathcal{F}^{-1}\left(f_{1}^{K_{1}} \star f_{2}^{K_{2}}\right)\right\}\right\|_{L^{2}} \\
\lesssim M_{3} \sum_{K_{3} \geqslant M_{3}} K_{3}^{-1 / 2} \beta_{M_{3}, K_{3}}^{b_{1}} \| \mathbb{1}_{D_{\lambda, M_{3}, \leqslant K_{3}} \cdot p \cdot f_{1}^{K_{1}} \star f_{2}^{K_{2}} \|_{L^{2}}}
\end{gathered}
$$

In particular, the first sum over the modulations $K_{3}<M_{3}$ is controlled by the first term in the second sum over the modulations $K_{3} \geqslant M_{3}$.

Finally, it suffices to show that $\forall K_{i} \geqslant\left(1 \vee M_{i}\right), i=1,2$,

$$
\begin{aligned}
M_{3} \sum_{K_{3} \geqslant M_{3}} K_{3}^{-1 / 2} \beta_{M_{3}, K_{3}}^{b_{1}}\left\|\mathbb{1}_{D_{\lambda, M_{3}, \leqslant K_{3}}} \cdot p \cdot f_{1}^{K_{1}} \star f_{2}^{K_{2}}\right\|_{L^{2}} \\
\quad \lesssim M_{1}^{1 / 2}\left(K_{1}^{1 / 2}\left\|p \cdot f_{1}^{K_{1}}\right\|_{L^{2}}\right)\left(K_{2}^{1 / 2}\left\|p \cdot f_{2}^{K_{2}}\right\|_{L^{2}}\right)
\end{aligned}
$$

Indeed, combining all the previous estimates, summing over $K_{i} \geqslant\left(1 \vee M_{i}\right)$ and using the definitions of $f_{i}^{K_{i}}(6.2),(6.3)$, the left-hand side of (6.1) is controled by

$$
M_{1}^{1 / 2}\left(\sum_{K_{1} \geqslant\left(1 \vee M_{1}\right)} K_{1}^{1 / 2}\left\|p \cdot f_{1}^{K_{1}}\right\|_{L^{2}}\right)\left(\sum_{K_{2} \geqslant M_{2}} K_{2}^{1 / 2}\left\|p \cdot f_{2}^{K_{2}}\right\|_{L^{2}}\right)
$$


The first sum is

$$
\begin{aligned}
\left(1 \vee M_{1}\right)^{1 / 2}\left\|\chi_{\left(1 \vee M_{1}\right)}(\tau-\omega) \mathcal{F}\left(\gamma_{\left(1 \vee M_{1}\right)^{-1}}\left(t-t_{M_{3}}\right) u_{M_{1}}\right)\right\|_{L^{2}} & \\
& +\sum_{K_{1}>\left(1 \vee M_{1}\right)} K_{1}^{1 / 2}\left\|p \cdot \rho_{K_{1}}(\tau-\omega) \mathcal{F}\left\{\gamma_{\left(1 \vee M_{1}\right)^{-1}}\left(t-t_{M_{3}}\right) u_{M_{1}}\right\}\right\|_{L^{2}}
\end{aligned}
$$

As $\chi \equiv 1$ on $\operatorname{supp} \gamma$, we have

$$
\gamma_{\left(1 \vee M_{1}\right)^{-1}}\left(t-t_{M_{3}}\right)=\gamma_{\left(1 \vee M_{1}\right)^{-1}}\left(t-t_{M_{3}}\right) \chi_{\left(1 \vee M_{1}\right)^{-1}}\left(t-t_{M_{3}}\right)
$$

so this term is controlled by $\left\|u_{M_{1}}\right\|_{F_{\lambda, M_{1}}^{0}}$ thanks to (3.10) and (3.11) with

$$
f=\mathcal{F}\left\{\chi_{\left(1 \vee M_{1}\right)^{-1}}\left(t-t_{M_{3}}\right) u_{M_{1}}\right\}
$$

and $K_{0}=\left(1 \vee M_{1}\right)$.

We can similarly bound the second sum by $\left\|v_{M_{2}}\right\|_{F_{\lambda, M_{2}}^{0}}$.

For now, we have established some estimates on expressions in the form $\int f_{1} \star f_{2} \cdot f_{3}$. Thus we first have to express $p \cdot f_{1} \star f_{2}$ according to $\left(p \cdot f_{1}\right)$ and $\left(p \cdot f_{2}\right)$. So, using the localizations in $\left|\xi_{i}\right|$ and the relation between the $M_{i}$, we can estimate

$$
\begin{aligned}
p\left(\xi_{1}+\xi_{2}, q_{1}+q_{2}\right) \sim 1+\frac{\left|q_{1}+q_{2}\right|}{\left(\xi_{1}+\xi_{2}\right)^{2}} & \\
& \lesssim 1+\frac{\left|q_{2}\right|}{\xi_{2}^{2}}+\frac{\left|\xi_{1}\right|\left\langle\xi_{1}\right\rangle}{\left(\xi_{1}+\xi_{2}\right)^{2}} \cdot \frac{\left|q_{1}\right|}{\left|\xi_{1}\right|\left\langle\xi_{1}\right\rangle} \\
& \lesssim p\left(\xi_{2}, q_{2}\right)+\frac{M_{1}\left(1 \vee M_{1}\right)}{M_{3}^{2}} p\left(\xi_{1}, q_{1}\right)
\end{aligned}
$$

We then treat separately the low and high frequency cases.

Case 1 : If $M_{1} \leqslant 1$.

We use the previous estimate to get

$$
\begin{aligned}
& \left\|\mathbb{1}_{D_{M_{3}, \leqslant K_{3}}} \cdot p \cdot f_{1}^{K_{1}} \star f_{2}^{K_{2}}\right\|_{L^{2}} \\
& \lesssim\left\|\mathbb{1}_{D_{\lambda, M_{3}, \leqslant K_{3}}} \cdot f_{1}^{K_{1}} \star\left(p \cdot f_{2}^{K_{2}}\right)\right\|_{L^{2}}+\frac{M_{1}\left(1 \vee M_{1}\right)}{M_{3}^{2}}\left\|\mathbb{1}_{D_{\lambda, M_{3}, \leqslant K_{3}}} \cdot\left(p \cdot f_{1}^{K_{1}}\right) \star f_{2}^{K_{2}}\right\|_{L^{2}} \\
& =I+I I
\end{aligned}
$$

To treat $I$, we use (5.30) :

$$
I \lesssim\left(1 \vee M_{1}\right) M_{1}^{1 / 2} K_{\min }^{1 / 2}\left\|p \cdot f_{1}^{K_{1}}\right\|_{L^{2}}\left\|p \cdot f_{2}^{K_{2}}\right\|_{L^{2}}
$$

Using that $K_{2} \geqslant M_{2} \sim M_{3}$, we obtain

$$
I \lesssim\left(K_{1} K_{2}\right)^{1 / 2} M_{1}^{1 / 2} M_{3}^{-1 / 2}\left\|p \cdot f_{1}^{K_{1}}\right\|_{L^{2}}\left\|p \cdot f_{2}^{K_{2}}\right\|_{L^{2}}
$$

Next, as we can exchange the roles played by $f_{1}$ and $f_{2}$ in (5.30), we can also apply this estimate to control $I I$ :

$$
I I \lesssim \frac{M_{1}\left(1 \vee M_{1}\right)}{M_{3}^{2}}\left(1 \vee M_{2}\right) M_{1}^{1 / 2} K_{m i n}^{1 / 2}\left\|p \cdot f_{1}^{K_{1}}\right\|_{L^{2}}\left\|p \cdot f_{2}^{K_{2}}\right\|_{L^{2}}
$$


Using that $M_{1} \leqslant 1 \leqslant M_{3} \sim M_{2}$, we directly get

$$
I I \lesssim M_{1}^{3 / 2} M_{3}^{-3 / 2}\left(K_{1} K_{2}\right)^{1 / 2}\left\|p \cdot f_{1}^{K_{1}}\right\|_{L^{2}}\left\|p \cdot f_{2}^{K_{2}}\right\|_{L^{2}}
$$

Finally

$$
\left\|\mathbb{1}_{D_{M_{3}, \leqslant K_{3}}} \cdot p \cdot f_{1}^{K_{1}} \star f_{2}^{K_{2}}\right\|_{L^{2}} \lesssim M_{1}^{1 / 2} M_{3}^{-1 / 2} \cdot K_{1}^{1 / 2}\left\|p \cdot f_{1}^{K_{1}}\right\|_{L^{2}} K_{2}^{1 / 2}\left\|p \cdot f_{2}^{K_{2}}\right\|_{L^{2}}
$$

so after summing

$$
\begin{aligned}
M_{3} \sum_{K_{3} \geqslant M_{3}} K_{3}^{-1 / 2} \beta_{M_{3}, K_{3}}^{b_{1}}\left\|\mathbb{1}_{D_{M_{3}, \leqslant K_{3}}} \cdot p \cdot f_{1}^{K_{1}} \star f_{2}^{K_{2}}\right\|_{L^{2}} & \\
& \lesssim M_{1}^{1 / 2} \cdot K_{1}^{1 / 2}\left\|p \cdot f_{1}^{K_{1}}\right\|_{L^{2}} K_{2}^{1 / 2}\left\|p \cdot f_{2}^{K_{2}}\right\|_{L^{2}}
\end{aligned}
$$

since

$$
\sum_{K_{3} \geqslant M_{3}} K_{3}^{-1 / 2} \beta_{M_{3}, K_{3}}^{b_{1}} \lesssim M_{3}^{-1 / 2}
$$

This is (6.4) in that case.

Case 2 : If $M_{1}>1$.

It is still sufficient to use (6.5) if $K_{3}$ is large enough .

Indeed, let us split the sum over $K_{3}$ in two parts, depending on wether $K_{3} \geqslant M_{1}^{2} M_{3}$ or $M_{3} \leqslant K_{3} \leqslant M_{1}^{2} M_{3}$.

Case 2.1 : If $K_{3} \geqslant M_{1}^{2} M_{3}$.

We proceed as in the case $M_{1} \leqslant 1$ to get

$$
\begin{aligned}
& \left\|\mathbb{1}_{D_{M_{3}, \leqslant K_{3}} \cdot p \cdot f_{1}^{K_{1}} \star f_{2}^{K_{2}}}\right\|_{L^{2}} \\
& \begin{aligned}
\lesssim\left\|\mathbb{1}_{D_{\lambda, M_{3}, \leqslant K_{3}}} \cdot f_{1}^{K_{1}} \star\left(p \cdot f_{2}^{K_{2}}\right)\right\|_{L^{2}}+\frac{M_{1}\left(1 \vee M_{1}\right)}{M_{3}^{2}}\left\|\mathbb{1}_{D_{\lambda, M_{3}, \leqslant K_{3}}} \cdot\left(p \cdot f_{1}^{K_{1}}\right) \star f_{2}^{K_{2}}\right\|_{L^{2}} \\
=I+I I
\end{aligned}
\end{aligned}
$$

As previously,

$$
\begin{aligned}
I \lesssim M_{1}^{3 / 2} K_{\min }^{1 / 2}\left\|p \cdot f_{1}^{K_{1}}\right\|_{L^{2}} \| p \cdot f_{2}^{K_{2}} & \|_{L^{2}} \\
& \lesssim M_{1}^{3 / 2}\left(K_{1} K_{2}\right)^{1 / 2} M_{2}^{-1 / 2}\left\|p \cdot f_{1}^{K_{1}}\right\|_{L^{2}}\left\|p \cdot f_{2}^{K_{2}}\right\|_{L^{2}}
\end{aligned}
$$

As for $I I$, we have again

$$
\begin{aligned}
I I \lesssim M_{1}^{5 / 2} M_{2} M_{3}^{-2} K_{m i n}^{1 / 2}\left\|p \cdot f_{1}^{K_{1}}\right\|_{L^{2}} & \left\|p \cdot f_{2}^{K_{2}}\right\|_{L^{2}} \\
& \lesssim M_{1}^{3 / 2}\left(K_{1} K_{2}\right)^{1 / 2} M_{2}^{-1 / 2}\left\|p \cdot f_{1}^{K_{1}}\right\|_{L^{2}}\left\|p \cdot f_{2}^{K_{2}}\right\|_{L^{2}}
\end{aligned}
$$

It remains to sum for the modulations $K_{3} \geqslant M_{1}^{2} M_{3}$ :

$$
\begin{aligned}
M_{3} \sum_{K_{3} \geqslant M_{1}^{2} M_{3}} K_{3}^{-1 / 2} \beta_{M_{3}, K_{3}}^{b_{1}} \| & \mathbb{1}_{D_{\lambda, M_{3}, \leqslant K_{3}}} \cdot p \cdot f_{1}^{K_{1}} \star f_{2}^{K_{2}} \|_{L^{2}} \\
& \lesssim M_{1}^{1 / 2}\left(K_{1}^{1 / 2}\left\|p \cdot f_{1}^{K_{1}}\right\|_{L^{2}}\right)\left(K_{2}^{1 / 2}\left\|p \cdot f_{2}^{K_{2}}\right\|_{L^{2}}\right)
\end{aligned}
$$


since

$$
\sum_{K_{3} \geqslant M_{1}^{2} M_{3}} K_{3}^{-1 / 2} \beta_{M_{3}, K_{3}}^{b_{1}} \lesssim M_{1}^{-1} M_{3}^{-1 / 2} \beta_{M_{3}, M_{1}^{2} M_{3}}^{b_{1}}
$$

and for $M_{1}>1$, we have $M_{1}^{2} M_{3}<M_{3}^{3}$ so $\beta_{M_{3}, M_{1}^{2} M_{3}}=1$.

Case 2.2 : If $M_{3} \leqslant K_{3} \leqslant M_{1}^{2} M_{3}$.

We improve (6.5) using the resonant function (cf. (5.9)). Observe that, since $\Omega\left(\zeta_{1}, \zeta_{2}, \zeta_{3}\right)$ and the hyperplane $\zeta_{1}+\zeta_{2}+\zeta_{3}=0$ are invariant under permutation, we have

$$
\left|\frac{q_{1}+q_{2}}{\xi_{1}+\xi_{2}}-\frac{q_{2}}{\xi_{2}}\right|=\left|\frac{\xi_{2}}{\xi_{1}\left(\xi_{1}+\xi_{2}\right)} \Omega\left(-\zeta_{1}-\zeta_{2}, \zeta_{2}, \zeta_{1}\right)+3 \xi_{1}^{2}\right|^{1 / 2}
$$

Since supp $f_{i} \subset D_{\lambda, M_{i}, \leqslant K_{i}}$ and $\int f_{1} \star f_{2} \cdot f_{3} \neq 0 \Rightarrow|\Omega| \lesssim K_{\max }$, we deduce the bound

$$
p\left(\xi_{1}+\xi_{2}, q_{1}+q_{2}\right) \lesssim 1+\frac{\left|q_{1}+q_{2}\right|}{\left|\xi_{1}+\xi_{2}\right|^{2}} \lesssim p\left(\xi_{2}, q_{2}\right)+M_{1}^{1 / 2} M_{3}^{-2} K_{\max }^{1 / 2}
$$

Therefore, we have the bound

$$
\begin{aligned}
& \left\|\mathbb{1}_{D_{\lambda, M_{3}, \leqslant K_{3}}} \cdot p \cdot f_{1}^{K_{1}} \star f_{2}^{K_{2}}\right\|_{L^{2}} \\
& \quad \lesssim\left\|\mathbb{1}_{D_{\lambda, M_{3}, \leqslant K_{3}}} \cdot f_{1}^{K_{1}} \star\left(p \cdot f_{2}^{K_{2}}\right)\right\|_{L^{2}}+M_{1}^{-1 / 2} M_{3}^{-1} K_{\max }^{1 / 2}\left\|\mathbb{1}_{D_{\lambda, M_{3}, \leqslant K_{3}}} \cdot f_{1}^{K_{1}} \star f_{2}^{K_{2}}\right\|_{L^{2}}
\end{aligned}
$$

as $M_{1} \lesssim M_{3}$.

To treat those terms, we distinguish the cases of corollary 5.9.

Case 2.1 (a) : If $K_{\max } \lesssim M_{1} M_{2} M_{3}$. In that case we estimate $K_{\max }^{1 / 2}$ in the second term and then apply (5.27) to both terms to get the bound

$$
\begin{aligned}
M_{3} \sum_{K_{3}=M_{3}}^{M_{1}^{2} M_{3}} K_{3}^{-1 / 2} \beta_{M_{3}, K_{3}}^{b_{1}}\left\|\mathbb{1}_{D_{\lambda, M_{3}} \leqslant K_{3}} \cdot p \cdot f_{1}^{K_{1}} \star f_{2}^{K_{2}}\right\|_{L^{2}} \\
\quad \lesssim \ln \left(M_{1}\right) M_{1}^{-1 / 2} \cdot\left(K_{1} K_{2}\right)^{1 / 2}\left\|p \cdot f_{1}^{K_{1}}\right\|_{L^{2}}\left\|p \cdot f_{2}^{K_{2}}\right\|_{L^{2}}
\end{aligned}
$$

Case $2.2(\mathrm{~b}) \&(\mathrm{c}):$ If $K_{\max } \gtrsim M_{1} M_{2} M_{3}$. Then we lose the factor $K_{\max }^{1 / 2}$ in the first term and use (5.7) for both terms with the indices corresponding to $K_{\min }$ and $K_{m e d}$, getting the final bound

$$
\begin{aligned}
M_{3} \sum_{K_{3}=M_{3}}^{M_{1}^{2} M_{3}} K_{3}^{-1 / 2} \beta_{M_{3}, K_{3}}^{b_{1}}\left\|\mathbb{1}_{D_{\lambda, M_{3}, \leqslant K_{3}}} \cdot p \cdot f_{1}^{K_{1}} \star f_{2}^{K_{2}}\right\|_{L^{2}} & \\
& \lesssim \ln \left(M_{1}\right) \cdot\left(K_{1} K_{2}\right)^{1 / 2}\left\|p \cdot f_{1}^{K_{1}}\right\|_{L^{2}}\left\|p \cdot f_{2}^{K_{2}}\right\|_{L^{2}}
\end{aligned}
$$




\section{Lemma $6.2($ High $\times$ High $\rightarrow$ Low $)$}

Let $M_{1}, M_{2}, M_{3} \in 2^{\mathbb{Z}}$ with $M_{1} \sim M_{2} \gtrsim\left(1 \vee M_{3}\right)$, and $b_{1} \in\left[0 ; 1 / 2\right.$. Then for $u_{M_{1}} \in$ $N_{\lambda, M_{1}}^{0}$ and $v_{M_{2}} \in N_{\lambda, M_{2}}^{0}$, we have

$$
\left.\left\|P_{M_{3}} \partial_{x}\left(u_{M_{1}} \cdot v_{M_{2}}\right)\right\|_{N_{\lambda, M_{3}}^{b_{1}}} \lesssim M_{2}^{3 / 2+4 b_{1}}\left(1 \vee M_{3}\right)^{-1}\left\|u_{M_{1}}||_{F_{\lambda, M_{1}}^{0}}\right\| v_{M_{2}}\right|_{F_{\lambda, M_{2}}^{0}}
$$

\section{Proof :}

We proceed similarly to the previous lemma, but this time the norm on the left-hand side only controls functions on time intervals of size $\left(1 \vee M_{3}\right)^{-1}$ whereas the norms on the righthand side require a control for time intervals of size $M_{2}^{-1}$. Thus will cut the time intervals in smaller pieces.

To do so, we take $\gamma$ as in the previous lemma. Since now $M_{1} \sim M_{2} \gtrsim\left(1 \vee M_{3}\right)$, we can write

$$
\begin{array}{r}
\chi_{\left(1 \vee M_{3}\right)^{-1}}\left(t-t_{M_{3}}\right)=\sum_{|m| \lesssim M_{2}\left(1 \vee M_{3}\right)^{-1}} \sum_{|n| \lesssim 100} \chi_{\left(1 \vee M_{3}\right)^{-1}}\left(t-t_{M_{3}}\right) \gamma_{M_{2}}\left(t-t_{M_{3}}-M_{2}^{-1} m\right) \\
\cdot \gamma_{M_{1}}\left(t-t_{M_{3}}-M_{2}^{-1} m-M_{1}^{-1} n\right)
\end{array}
$$

As previously, without loss of generality, we can assume $m=n=0$, and defining

$$
f_{1}:=\mathcal{F}\left\{\gamma\left(M_{1}\left(t-t_{M_{3}}\right)\right) u_{M_{1}}\right\}
$$

and

$$
f_{2}:=\mathcal{F}\left\{\gamma\left(M_{2}\left(t-t_{M_{3}}\right)\right) v_{M_{2}}\right\}
$$

it then suffices to prove that $\forall K_{i} \geqslant\left(1 \vee M_{i}\right)$ :

$$
\begin{aligned}
M_{2}\left(1 \vee M_{3}\right)^{-1} \cdot M_{3} \sum_{K_{3} \geqslant\left(1 \vee M_{3}\right)} & K_{3}^{-1 / 2} \beta_{M_{3}, K_{3}}^{b_{1}}\left\|\mathbb{1}_{D_{\lambda, M_{3}, \leqslant K_{3}} \cdot p \cdot f_{1}^{K_{1}} \star f_{2}^{K_{2}}}\right\|_{L^{2}} \\
& \lesssim M_{2}^{2}\left(1 \vee M_{3}\right)^{-1} K_{1}^{1 / 2}\left\|p \cdot f_{1}^{K_{1}}\right\|_{L^{2}} K_{2}^{1 / 2}\left\|p \cdot f_{2}^{K_{2}}\right\|_{L^{2}}
\end{aligned}
$$

where we have denoted

$$
f_{i}^{M_{2}}:=\chi_{M_{i}}(\tau-\omega) f_{i} \text { and } f_{i}^{K_{i}}:=\rho_{K_{i}}(\tau-\omega) f_{i}, K_{i}>M_{i}
$$

As previously, we need to estime $p\left(\xi_{1}+\xi_{2}, q_{1}+q_{2}\right)$ with respect to $p\left(\xi_{1}, q_{1}\right)$ and $p\left(\xi_{2}, q_{2}\right)$ :

$$
\begin{aligned}
p\left(\xi_{1}+\xi_{2}, q_{1}+\right. & \left.q_{2}\right) \lesssim 1+\frac{\left|q_{1}+q_{2}\right|}{\left|\xi_{1}+\xi_{2}\right|\left\langle\xi_{1}+\xi_{2}\right\rangle} \\
& \begin{aligned}
\lesssim 1+\frac{\left|\xi_{1}\right|\left\langle\xi_{1}\right\rangle}{\left|\xi_{1}+\xi_{2}\right|\left\langle\xi_{1}+\xi_{2}\right\rangle} & \frac{\left|q_{1}\right|}{\left|\xi_{1}\right|\left\langle\xi_{1}\right\rangle}+\frac{\left|\xi_{2}\right|\left\langle\xi_{2}\right\rangle}{\left|\xi_{1}+\xi_{2}\right|\left\langle\xi_{1}+\xi_{2}\right\rangle} \frac{\left|q_{2}\right|}{\left|\xi_{2}\right|\left\langle\xi_{2}\right\rangle} \\
& \lesssim M_{2}^{2} M_{3}^{-1}\left(1 \vee M_{3}\right)^{-1}\left(p\left(\xi_{1}, q_{1}\right)+p\left(\xi_{2}, q_{2}\right)\right)
\end{aligned}
\end{aligned}
$$

Just as before, we distinguish several cases.

Case 1.1 : If $M_{3} \leqslant 1$ and $K_{3} \geqslant M_{2}^{5}$ : 
We use (6.10), so that the left-hand side of (6.9) is controled with

$$
\begin{aligned}
M_{2}^{3} \sum_{K_{3} \geqslant M_{2}^{5}} K_{3}^{b_{1}-1 / 2}\left\{\| \mathbb{1}_{D_{\lambda, M_{3}, \leqslant K_{3}}} \cdot\left(p \cdot f_{1}^{K_{1}}\right) \star f_{2}^{K_{2}}\right. & \|_{L^{2}} \\
& \left.+\left\|\mathbb{1}_{D_{\lambda, M_{3}, \leqslant K_{3}} \cdot f_{1}^{K_{1}} \star\left(p \cdot f_{2}^{K_{2}}\right)}\right\|_{L^{2}}\right\}
\end{aligned}
$$

Using (5.30) and that $M_{1} \sim M_{2} \geqslant 1$ and $K_{1}, K_{2} \gtrsim M_{2}$, we get the bound

$$
\begin{aligned}
\sum_{K_{3} \geqslant M_{2}^{5}} K_{3}^{b_{1}-1 / 2} M_{2}^{3} \cdot M_{2} M_{3}^{1 / 2} K_{m i n}^{1 / 2} & \left\|p \cdot f_{1}^{K_{1}}\right\|_{L^{2}}\left\|p \cdot f_{2}^{K_{2}}\right\|_{L^{2}} \\
& \lesssim M_{2}^{1+5 b_{1}} M_{3}^{1 / 2} \cdot\left(K_{1} K_{2}\right)^{1 / 2}\left\|p \cdot f_{1}^{K_{1}}\right\|_{L^{2}}\left\|p \cdot f_{2}^{K_{2}}\right\|_{L^{2}}
\end{aligned}
$$

which suffices for (6.9).

Case 1.2 : If $M_{3} \leqslant 1$ and $1 \leqslant K_{3} \leqslant M_{2}^{5}$ :

We improve the control on $p$ in this regime by using $\Omega$ as in (6.7). We get in this case

$$
\left|\frac{q_{1}+q_{2}}{\xi_{1}+\xi_{2}}-\frac{q_{1}}{\xi_{1}}\right|=\left|\frac{\xi_{2}}{\xi_{1}\left(\xi_{1}+\xi_{2}\right)} \Omega\left(\zeta_{1},-\zeta_{1}-\zeta_{2}, \zeta_{2}\right)+3 \xi_{2}^{2}\right|^{1 / 2} \lesssim M_{2}+M_{3}^{-1 / 2} K_{\max }^{1 / 2}
$$

from which we deduce

$$
p\left(\xi_{1}+\xi_{2}, q_{1}+q_{2}\right) \lesssim M_{2} p\left(\xi_{1}, q_{1}\right)+M_{3}^{-1 / 2} K_{\max }^{1 / 2}
$$

Using this estimate, we get the bound

$$
\begin{aligned}
M_{3} M_{2}^{2} \sum_{K_{3}=1}^{M_{2}^{5}} K_{3}^{b_{1}-1 / 2}\left\{\left\|\mathbb{1}_{D_{\lambda, M_{3}, \leqslant K_{3}}} \cdot\left(p \cdot f_{1}^{K_{1}}\right) \star f_{2}^{K_{2}}\right\|_{L^{2}}\right. \\
\quad+M_{3}^{-1 / 2} M_{2}^{-1} K_{\text {max }}^{1 / 2} \| \mathbb{1}_{\left.D_{\lambda, M_{3}, \leqslant K_{3}} \cdot f_{1}^{K_{1}} \star f_{2}^{K_{2}} \|_{L^{2}}\right\}}
\end{aligned}
$$

Observe that the term within the braces is the same as in case 2.2 of lemma 6.1 , so we control it the exact same way to get the final bound

$$
M_{3}^{1 / 2} M_{2}^{1+5 b_{1}} \cdot\left(K_{1} K_{2}\right)^{1 / 2}\left\|p \cdot f_{1}^{K_{1}}\right\|_{L^{2}}\left\|p \cdot f_{2}^{K_{2}}\right\|_{L^{2}}
$$

Case 2.1 : If $M_{3} \geqslant 1$ and $K_{3} \geqslant M_{2}^{4} M_{3}^{-1}$.

We use again (6.10) so that the left-hand side of (6.9) is controled with

$$
\begin{aligned}
M_{2} \sum_{K_{3} \geqslant M_{2}^{4} M_{3}^{-1}} K_{3}^{-1 / 2} \beta_{M_{3}, K_{3}}^{b_{1}} M_{2}^{2} M_{3}^{-2}\left\{\left\|\mathbb{1}_{D_{\lambda, M_{3}, \leqslant K_{3}}} \cdot\left(p \cdot f_{1}^{K_{1}}\right) \star f_{2}^{K_{2}}\right\|_{L^{2}}\right. \\
+\| \mathbb{1}_{\left.D_{\lambda, M_{3}, \leqslant K_{3}} \cdot f_{1}^{K_{1}} \star\left(p \cdot f_{2}^{K_{2}}\right) \|_{L^{2}}\right\}}
\end{aligned}
$$

With (5.30) again, we obtain the bound

$$
\begin{aligned}
\sum_{K_{3} \geqslant M_{2}^{4} M_{3}^{-1}} K_{3}^{-1 / 2} \beta_{M_{3}, K_{3}}^{b_{1}} M_{2}^{3} M_{3}^{-2} \cdot M_{2} M_{3}^{1 / 2} K_{\min }^{1 / 2}\left\|p \cdot f_{1}^{K_{1}}\right\|_{L^{2}}\left\|p \cdot f_{2}^{K_{2}}\right\|_{L^{2}} \\
\lesssim M_{2}^{3 / 2+4 b_{1}} M_{3}^{-1-4 b_{1}} \cdot\left(K_{1} K_{2}\right)^{1 / 2}\left\|p \cdot f_{1}^{K_{1}}\right\|_{L^{2}} K_{2}^{1 / 2}\left\|p \cdot f_{2}^{K_{2}}\right\|_{L^{2}}
\end{aligned}
$$


Case 2.2 : If $M_{3} \geqslant 1$ and $M_{3} \leqslant K_{3} \leqslant M_{2}^{4} M_{3}^{-1}$.

(6.11) becomes in this case

$$
p\left(\xi_{1}+\xi_{2}, q_{1}+q_{2}\right) \lesssim M_{3}^{-1} M_{2} p\left(\xi_{1}, q_{1}\right)+M_{3}^{-3 / 2} K_{\max }^{1 / 2}
$$

So the use of (6.12) allows us to bound the left-hand side of (6.9) with

$$
\begin{aligned}
M_{2}^{2} M_{3}^{-1} \sum_{K_{3}=M_{3}}^{M_{2}^{4} M_{3}^{-1}} K_{3}^{-1 / 2} \beta_{M_{3}, K_{3}}^{b_{1}}\left\{\left\|\mathbb{1}_{D_{\lambda, M_{3}, \leqslant K_{3}}} \cdot\left(p \cdot f_{1}^{K_{1}}\right) \star f_{2}^{K_{2}}\right\|_{L^{2}}\right. \\
\left.+M_{3}^{-1 / 2} M_{2}^{-1} K_{\max }^{1 / 2}\left\|\mathbb{1}_{D_{\lambda, M_{3}, \leqslant K_{3}} \cdot f_{1}^{K_{1}} \star f_{2}^{K_{2}}}\right\|_{L^{2}}\right\}
\end{aligned}
$$

Proceeding similarly to the previous cases, we finally obtain the bound

$$
M_{2}^{1+4 b_{1}} M_{3}^{-1-4 b_{1}} \cdot\left(K_{1} K_{2}\right)^{1 / 2}\left\|p \cdot f_{1}^{K_{1}}\right\|_{L^{2}} K_{2}^{1 / 2}\left\|p \cdot f_{2}^{K_{2}}\right\|_{L^{2}}
$$

\section{Lemma $6.3($ Low $\times$ Low $\rightarrow$ Low $)$}

Let $M_{1}, M_{2}, M_{3} \in 2^{-\mathbb{Z}}$ and $b_{1} \in\left[0 ; 1 / 2\left[\right.\right.$. Then for $u_{M_{1}} \in F_{\lambda, M_{1}}^{0}$ and $v_{M_{2}} \in F_{\lambda, M_{2}}^{0}$ we have

$$
\left\|\left.P_{M_{3}} \partial_{x}\left(u_{M_{1}} \cdot v_{M_{2}}\right)\right|_{N_{\lambda, M_{3}}^{b_{1}}} \lesssim\left(M_{1} M_{2} M_{3}\right)^{1 / 2}\right\| u_{M_{1}}\left\|_{F_{\lambda, M_{1}}^{0}}\right\| v_{M_{2}} \|_{F_{\lambda, M_{2}}^{0}}
$$

Proof :

As in the previous lemmas, it is enough to prove that $\forall K_{1}, K_{2} \geqslant 1$,

$$
\begin{aligned}
M_{3} \sum_{K_{3} \geqslant 1} K_{3}^{-1 / 2} \beta_{M_{3}, K_{3}}^{b_{1}}\left\|\mathbb{1}_{D_{\lambda, M_{3}, \leqslant K_{3}}} \cdot p \cdot f_{1}^{K_{1}} \star f_{2}^{K_{2}}\right\|_{L^{2}} & \\
& \lesssim K_{1}^{1 / 2}\left\|p \cdot f_{1}^{K_{1}}\right\|_{L^{2}} K_{2}^{1 / 2}\left\|p \cdot f_{2}^{K_{2}}\right\|_{L^{2}}
\end{aligned}
$$

By symmetry, we may assume $M_{1} \leqslant M_{2}$, so similarly to (6.10), we have in this case

$$
p\left(\xi_{1}+\xi_{2}, q_{1}+q_{2}\right) \lesssim M_{2} M_{3}^{-1}\left(p\left(\xi_{1}, q_{1}\right)+p\left(\xi_{2}, q_{2}\right)\right)
$$

It then suffices to use (5.30) along with the previous bound to get (6.14) :

$$
\begin{aligned}
& M_{3} \sum_{K_{3} \geqslant 1} K_{3}^{-1 / 2} \beta_{M_{3}, K_{3}}^{b_{1}}\left\|\mathbb{1}_{D_{\lambda, M_{3}, \leqslant K_{3}}} \cdot p \cdot f_{1}^{K_{1}} \star f_{2}^{K_{2}}\right\|_{L^{2}} \\
& \lesssim M_{2} \sum_{K_{3} \geqslant 1} K_{3}^{b_{1}-1 / 2} M_{\min }^{1 / 2} K_{\min }^{1 / 2}\left\|p \cdot f_{1}^{K_{1}}\right\|_{L^{2}}\left\|p \cdot f_{2}^{K_{2}}\right\|_{L^{2}} \\
& \quad \lesssim\left(M_{1} M_{2} M_{3}\right)^{1 / 2} \cdot\left(K_{1} K_{2}\right)^{1 / 2}\left\|p \cdot f_{1}^{K_{1}}\right\|_{L^{2}}\left\|p \cdot f_{2}^{K_{2}}\right\|_{L^{2}}
\end{aligned}
$$




\section{Proposition 6.4}

Let $T \in] 0 ; 1], \alpha \geqslant 1$ and $b_{1} \in[0 ; 1 / 8]$. Then for $u, v \in \mathbf{F}_{\lambda}^{\alpha, 0}(T)$ we have

$$
\left.\left\|\left.\partial_{x}(u v)\right|_{\mathbf{N}_{\lambda}^{\alpha, b_{1}}(T)} \lesssim\right\| u\right|_{\mathbf{F}_{\lambda}^{\alpha, 0}(T)}\|v\|_{\mathbf{F}_{\lambda}^{1,0}(T)}+\left\|\left.u\right|_{\mathbf{F}_{\lambda}^{1,0}(T)}|| v\right\|_{\mathbf{F}_{\lambda}^{\alpha, 0}(T)}
$$

Proof :

For $M_{1} \in 2^{\mathbb{Z}}$, let us choose an extension $u_{M_{1}} \in F_{\lambda, M_{1}}^{0}$ of $P_{M_{1}} u$ satisfying

$$
\left\|u_{M_{1}}\right\|_{F_{\lambda, M_{1}}^{0}} \leqslant 2\left\|P_{M_{1}} u\right\|_{F_{\lambda, M_{1}}^{0}(T)}
$$

and let us define $v_{M_{2}}$ analogously.

Using the definition of $\mathbf{F}_{\lambda}^{\alpha, b_{1}}(T)(3.2)$ and $\mathbf{N}_{\lambda}^{\alpha, b_{1}}(T)$ (3.3), it then suffices to show that

$$
\begin{aligned}
& \sum_{M_{1}, M_{2}, M_{3}}\left(1 \vee M_{3}\right)^{2 \alpha}\left\|P_{M_{3}} \partial_{x}\left(u_{M_{1}} \cdot v_{M_{2}}\right)\right\|_{N_{\lambda, M_{3}}^{b_{1}}}^{2} \\
& \lesssim \sum_{M_{1}, M_{2}}\left\{\left(1 \vee M_{1}\right)^{2 \alpha}\left\|u_{M_{1}}\right\|_{F_{\lambda, M_{1}}^{0}}^{2}\left(1 \vee M_{2}\right)^{2}\left\|v_{M_{2}}\right\|_{F_{\lambda, M_{2}}^{0}}^{2}\right. \\
&\left.\quad+\left(1 \vee M_{1}\right)^{2}\left\|u_{M_{1}}\right\|_{F_{\lambda, M_{1}}^{0}}^{2}\left(1 \vee M_{2}\right)^{2 \alpha}\left\|v_{M_{2}}\right\|_{F_{\lambda, M_{2}}^{0}}^{2}\right\}
\end{aligned}
$$

Since the left-hand side of (6.15) is symmetrical in $u$ and $v$, we can assume $M_{1} \leqslant M_{2}$.

Then we can decompose the left-hand side of (6.16) depending on the relation between $M_{1}, M_{2}$ and $M_{3}$ :

$$
\begin{aligned}
\sum_{M_{1}, M_{2}, M_{3}>0}\left(1 \vee M_{3}\right)^{2 \alpha}\left\|P_{M_{3}} \partial_{x}\left(u_{M_{1}} \cdot v_{M_{2}}\right)\right\|_{N_{\lambda, M_{3}}^{b_{1}}}^{2} & \\
= & \sum_{i=1}^{3} \sum_{\left(M_{1}, M_{2}, M_{3}\right) \in A_{i}}\left(1 \vee M_{3}\right)^{2 \alpha}\left\|P_{M_{3}} \partial_{x}\left(u_{M_{1}} \cdot v_{M_{2}}\right)\right\|_{N_{\lambda, M_{3}}^{b_{1}}}^{2}
\end{aligned}
$$

where

$$
\left\{\begin{array}{l}
A_{1}:=\left\{\left(M_{1}, M_{2}, M_{3}\right) \in 2^{\mathbb{Z}},\left(1 \vee M_{1}\right) \lesssim M_{2} \sim M_{3}\right\} \\
A_{2}:=\left\{\left(M_{1}, M_{2}, M_{3}\right) \in 2^{\mathbb{Z}},\left(1 \vee M_{3}\right) \lesssim M_{1} \sim M_{2}\right\} \\
A_{3}:=\left\{\left(M_{1}, M_{2}, M_{3}\right) \in 2^{\mathbb{Z}}, M_{\max } \lesssim 1\right\}
\end{array}\right.
$$

Using lemma 6.1, the first term is estimated by

$$
\begin{aligned}
\sum_{\left(M_{1}, M_{2}, M_{3}\right) \in A_{1}}\left(1 \vee M_{3}\right)^{2 \alpha}\left\|P_{M_{3}} \partial_{x}\left(u_{M_{1}} \cdot v_{M_{2}}\right)\right\|_{N_{\lambda, M_{3}}^{b_{1}}}^{2} & \\
& \lesssim \sum_{M_{2} \gtrsim 1} \sum_{M_{1} \lesssim M_{2}} M_{1}\left(1 \vee M_{2}\right)^{2 \alpha}\left\|u_{M_{1}}\right\|_{F_{\lambda, M_{1}}^{0}}^{2}\left\|v_{M_{2}}\right\|_{F_{\lambda, M_{2}}^{0}}^{2}
\end{aligned}
$$

which suffices for (6.16). For the second term, the use of lemma 6.2 provides the bound

$$
\begin{aligned}
\sum_{\left(M_{1}, M_{2}, M_{3}\right) \in A_{2}}\left(1 \vee M_{3}\right)^{2 \alpha}\left\|P_{M_{3}} \partial_{x}\left(u_{M_{1}} \cdot v_{M_{2}}\right)\right\|_{N_{\lambda, M_{3}}^{b_{1}}}^{2} & \\
& \lesssim \sum_{M_{2} \gtrsim 1} \sum_{M_{1} \sim M_{2}} M_{2}^{3+8 b_{1}+2(\alpha-1)}\left\|u_{M_{1}}\right\|_{F_{\lambda, M_{1}}^{0}}^{2}\left\|v_{M_{2}}\right\|_{F_{\lambda, M_{2}}^{0}}^{2}
\end{aligned}
$$


which is enough for (6.16) since $b_{1} \in[0 ; 1 / 8]$. Finally, lemma 6.3 allows us to control the last term by

$$
\begin{aligned}
\sum_{\left(M_{1}, M_{2}, M_{3}\right) \in A_{3}}\left(1 \vee M_{3}\right)^{2 \alpha}\left\|P_{M_{3}} \partial_{x}\left(u_{M_{1}} \cdot v_{M_{2}}\right)\right\|_{N_{\lambda, M_{3}}^{b_{1}}}^{2} & \\
& \lesssim \sum_{M_{1} \in 2^{-N}} \sum_{M_{2} \in 2^{-N}} M_{1} M_{2}\left\|u_{M_{1}}\right\|_{F_{\lambda, M_{1}}^{0}}^{2}\left\|v_{M_{2}}\right\|_{F_{\lambda, M_{2}}^{0}}^{2}
\end{aligned}
$$

which concludes the proof of the bilinear estimate.

\subsection{For the difference equation}

The end of this section is devoted to treating (1.15). Let $b_{1} \in[0 ; 1 / 2[$.

We begin with the low frequency interactions :

Lemma $6.5($ Low $\times$ Low $\rightarrow$ Low $)$

Let $M_{1}, M_{2}, M_{3} \in 2^{-\mathbb{Z}}$. Then for $u_{M_{1}} \in \overline{F_{\lambda, M_{1}}^{0}}$ and $v_{M_{2}} \in F_{\lambda, M_{2}}^{0}$, we have

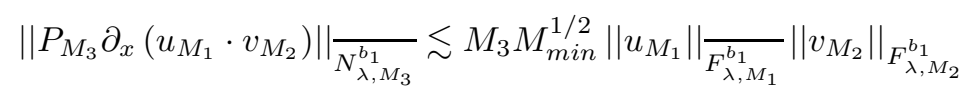

\section{Proof :}

Proceeding as for the previous lemmas, it suffices to prove that for all $K_{1}, K_{2} \geqslant 1$ and $f_{i}^{K_{i}}: D_{\lambda, M_{i}, \leqslant K_{i}} \rightarrow \mathbb{R}_{+}$,

$$
\begin{aligned}
M_{3} \sum_{K_{3} \geqslant 1} K_{3}^{-1 / 2} \beta M_{3}, K_{3}{ }^{b_{1}}\left\|\mathbb{1}_{D_{\lambda, M_{3}, \leqslant K_{3}}} \cdot f_{1}^{K_{1}} \star f_{2}^{K_{2}}\right\|_{L^{2}} & \\
& \lesssim M_{3} M_{\text {min }}^{1 / 2} \cdot\left(K_{1} K_{2}\right)^{1 / 2}\left\|f_{1}^{K_{1}}\right\|_{L^{2}}\left\|p \cdot f_{2}^{K_{2}}\right\|_{L^{2}}
\end{aligned}
$$

This follows directly from (5.30).

\section{Lemma 6.6 $($ High $\times$ High $\rightarrow$ Low $)$}

Let $M_{1}, M_{2}, M_{3} \in 2^{\mathbb{Z}}$ with $M_{1} \sim M_{2}$
$F_{\lambda, M_{2}}^{0}$, we have
$\| P_{M_{3}} \partial_{x}\left(u_{M_{1}} \cdot v_{M_{2}}\right) \mid \frac{}{N_{\lambda, M_{3}}^{b_{1}}} \lesssim\left(1 \wedge M_{3}\right)$. Then for $u_{M_{1}} \in \overline{F_{\lambda, M_{1}}^{0}}$ and $v_{M_{2}} \in$

\section{Proof :}

Following the proof of lemma 6.2 , it is enough to prove that for all $K_{i} \geqslant\left(1 \vee M_{i}\right)$ and 


$$
\begin{aligned}
& f_{i}^{K_{i}}: D_{\lambda, M_{i}, \leqslant K_{i}} \rightarrow \mathbb{R}_{+} \\
& M_{3} M_{2}\left(1 \vee M_{3}\right)^{-1} \sum_{K_{3} \geqslant\left(1 \vee M_{3}\right)} K_{3}^{-1 / 2} \beta M_{3}, K_{3}{ }^{b_{1}}\left\|\mathbb{1}_{D_{\lambda, M_{3}, \leqslant K_{3}}} \cdot f_{1}^{K_{1}} \star f_{2}^{K_{2}}\right\|_{L^{2}} \\
& \quad \lesssim\left(1 \wedge M_{3}\right)^{3 / 2} M_{2} \cdot\left(K_{1} K_{2}\right)^{1 / 2}\left\|f_{1}^{K_{1}}\right\|_{L^{2}}\left\|p \cdot f_{2}^{K_{2}}\right\|_{L^{2}}
\end{aligned}
$$

This is a consequence of (5.7).

It remains to treat the interaction between low and high frequencies. Since $u$ and $v$ do not play a symetric role anymore, we have to distinguih which one has the low frequency part.

Lemma $6.7($ Low $\times$ High $\rightarrow$ High $)$

$$
\begin{aligned}
& \text { Let }\left(1 \vee M_{1}\right) \lesssim M_{2} \sim M_{3} \text { and } u_{M_{1}} \in \overline{F_{\lambda, M_{1}}^{0}}, v_{M_{2}} \in F_{\lambda, M_{2}}^{0} \text {. Then } \\
& \qquad\left\|P_{M_{3}} \partial_{x}\left(u_{M_{1}} \cdot v_{M_{2}}\right) \mid \frac{}{N_{\lambda, M_{3}}^{b_{1}}} \lesssim M_{1}^{1 / 2}\left(1 \vee M_{1}\right)^{1 / 4} M_{2}^{1 / 4}\right\| u_{M_{1}}\left\|_{\overline{F_{\lambda, M_{1}}^{0}}}\right\| v_{M_{2}} \|_{F_{\lambda, M_{2}}^{0}}
\end{aligned}
$$

Proof :

Foolowing the proof of lemma 6.1 , it suffices to prove that for all $K_{i} \geqslant\left(1 \vee M_{i}\right)$ and $f_{i}^{K_{i}}: D_{\lambda, M_{i}, \leqslant K_{i}} \rightarrow \mathbb{R}_{+}$,

$$
\begin{aligned}
M_{3} \sum_{K_{3} \geqslant M_{3}} K_{3}^{-1 / 2} \beta M_{3}, K_{3}{ }^{b_{1}} & \left\|\mathbb{1}_{D_{\lambda, M_{3}}, \leqslant K_{3}} \cdot f_{1}^{K_{1}} \star f_{2}^{K_{2}}\right\|_{L^{2}} \\
& \left.\lesssim M_{1}^{1 / 2}\left(1 \vee M_{1}\right)^{1 / 4} M_{2}^{1 / 4} \cdot\left(K_{1} K_{2}\right)^{1 / 2}\left\|f_{1}^{K_{1}}\right\|\right|_{L^{2}}\left\|p \cdot f_{2}^{K_{2}}\right\|_{L^{2}}
\end{aligned}
$$

Again, this follows from using (5.7).

\section{Lemma $6.8($ High $\times$ Low $\rightarrow$ High $)$}

$$
\begin{aligned}
& \text { Let }\left(1 \vee M_{2}\right) \lesssim M_{1} \sim M_{3} \text { and } u_{M_{1}} \in \overline{F_{\lambda, M_{1}}^{0}}, v_{M_{2}} \in F_{\lambda, M_{2}}^{0} \text {. Then } \\
& \left\|P_{M_{3}} \partial_{x}\left(u_{M_{1}} \cdot v_{M_{2}}\right)\right\|_{\overline{N_{\lambda, M_{3}}^{0}}} \lesssim\left(1 \vee M_{2}\right)\left\|u_{M_{1}}\right\|_{\overline{F_{\lambda, M_{1}}^{0}}}\left\|v_{M_{2}}\right\|_{F_{\lambda, M_{2}}^{0}}
\end{aligned}
$$

Proof :

As previously, it is enough to prove

$$
\begin{aligned}
M_{3} \sum_{K_{3} \geqslant M_{3}} K_{3}^{-1 / 2}\left\|\mathbb{1}_{D_{\lambda, M_{3}, \leqslant K_{3}} \cdot f_{1}^{K_{1}} \star f_{2}^{K_{2}}}\right\|_{L^{2}} & \\
& \lesssim\left(1 \vee M_{2}\right) \cdot\left(K_{1} K_{2}\right)^{1 / 2}\left\|f_{1}^{K_{1}}\right\|\left\|_{L^{2}}\right\| p \cdot f_{2}^{K_{2}} \|_{L^{2}}
\end{aligned}
$$

for $K_{i} \geqslant\left(1 \vee M_{i}\right)$ and $f_{i}^{K_{i}}: D_{\lambda, M_{i}, \leqslant K_{i}} \rightarrow \mathbb{R}_{+}$.

Following the proof of lemma 6.1 ,we distinguish several cases. 
Case $1:$ If $M_{2} \leqslant 1$.

This is a consequence of (5.30).

Case 2 : If $M_{2} \geqslant 1$.

We split the sum over $K_{3}$ into two parts. The high modulations part $K_{3} \geqslant M_{2} M_{3}$ is treated again with (5.30), whereas for the sum over the modulations $M_{3} \leqslant K_{3} \leqslant M_{2} M_{3}$ is controled by using (5.28) (which is the worst case of corollary 5.9).

We finally combine the previous estimates to get

\section{Proposition 6.9}

Let $T \in] 0 ; 1], b_{1} \in\left[0 ; 1 / 2\left[\right.\right.$ and $u \in \overline{\mathbf{F}}^{0}(T), v \in \mathbf{F}_{\lambda}^{1,0}(T)$. Then

$$
\left.\left\|\left.\partial_{x}(u v)\right|_{{\overline{\mathbf{N}_{\lambda}}}^{b_{1}}(T)} \lesssim\right\| u\right|_{\overline{\mathbf{F}}_{\lambda}^{0}(T)}\|v\|_{\mathbf{F}_{\lambda}^{1,0}(T)}
$$

Proof :

First, for $M_{1}, M_{2} \in 2^{\mathbb{Z}}$, we fix an extension $u_{M_{1}} \in \overline{F_{\lambda, M_{1}}^{0}}$ of $P_{M_{1}} u$ to $\mathbb{R}$ satisfying

$$
\left\|u_{M_{1}}\right\|_{\overline{F_{\lambda, M_{1}}}} \leqslant 2\left\|P_{M_{1}} u\right\|_{\overline{F_{\lambda, M_{1}}^{0}}(T)}
$$

and similarly for $v_{M_{2}}$.

Using the definition of $\overline{\mathbf{F}}_{\lambda}^{0}$ (3.7) and ${\overline{\mathbf{N}_{\lambda}}}^{b_{1}}$ (3.8), it then suffices to show that

$$
\begin{aligned}
\sum_{M_{1}, M_{2}, M_{3} \in 2^{\mathbb{Z}}}\left\|\partial_{x}\left(u_{M_{1}} \cdot v_{M_{2}}\right)\right\| & \frac{2}{N_{\lambda, M_{3}}^{b_{1}}(T)} \\
& \lesssim \sum_{M_{1}, M_{2} \in 2^{\mathbb{Z}}}\left\|u_{M_{1}}\right\|_{F_{\lambda, M_{1}}^{0}}^{2}(T)
\end{aligned}
$$

As in the proof of proposition 6.4, we separate 4 cases, so it suffices to show that for $i \in\{1,2,3,4\}$,

$$
\begin{aligned}
\sum_{\left(M_{1}, M_{2}, M_{3}\right) \in B_{i}}\left\|\partial_{x}\left(u_{M_{1}} \cdot v_{M_{2}}\right)\right\| \frac{\|_{N_{\lambda, M_{3}}^{b_{1}}}^{2}(T)}{} & \\
& \lesssim \sum_{M_{1}, M_{2} \in 2^{\mathbb{Z}}}\left\|u_{M_{1}}\right\| \frac{2}{F_{\lambda, M_{1}}^{0}(T)}\left(1 \vee M_{2}\right)^{2}\left\|v_{M_{2}}\right\|_{F_{\lambda, M_{2}}^{0}}^{2}(T)
\end{aligned}
$$

with

$$
\left\{\begin{array}{l}
B_{1}:=\left\{\left(M_{1}, M_{2}, M_{3}\right) \in 2^{-\mathbb{Z}}\right\} \\
B_{2}:=\left\{\left(M_{1}, M_{2}, M_{3}\right) \in 2^{\mathbb{Z}}, M_{1} \sim M_{2} \gtrsim\left(1 \vee M_{3}\right)\right\} \\
B_{3}:=\left\{\left(M_{1}, M_{2}, M_{3}\right) \in 2^{\mathbb{Z}}, M_{2} \sim M_{3} \gtrsim\left(1 \vee M_{1}\right)\right\} \\
B_{4}:=\left\{\left(M_{1}, M_{2}, M_{3}\right) \in 2^{\mathbb{Z}}, M_{1} \sim M_{3} \gtrsim\left(1 \vee M_{2}\right)\right\}
\end{array}\right.
$$

This follows from lemmas $6.5,6.6,6.7$, and 6.8 respectively. 


\section{$7 \quad$ Energy estimates}

In this section we prove the energy estimates (1.13) and (1.16). As the nonlinear term is expressed as a bilinear form, we will need some control on trilinear form to deal with the energy estimate :

\section{Lemma 7.1}

Let $T \in\left[0 ; 1\left[, M_{1}, M_{2}, M_{3} \in 2^{\mathbb{Z}}\right.\right.$ with $M_{\max } \geqslant 1$, and $b_{1} \in[0 ; 1 / 8]$. Then for $u_{i} \in \overline{F_{\lambda, M_{i}}^{b_{1}}}(T), i \in\{1,2,3\}$, with one of them in $F_{\lambda, M_{i}}^{b_{1}}(T)$ (in order for the integral to converge), we have

$$
\left.\left|\int_{[0, T] \times \mathbb{R} \times \mathbb{T}_{\lambda}} u_{1} u_{2} u_{3} \mathrm{~d} t \mathrm{~d} x \mathrm{~d} y\right| \lesssim \Lambda_{b_{1}}\left(M_{\min }, M_{\max }\right) \prod_{i=1}^{3}\left\|u_{i}\right\|\right|_{\overline{F_{\lambda, M_{i}}}(T)}
$$

where

$$
\Lambda_{b_{1}}(X, Y)=\left(X \wedge X^{-1}\right)^{1 / 2}+\left(\frac{(1 \vee X)}{Y}\right)^{2 b_{1}}
$$

\section{Proof :}

Using the symmetry property (5.11), we may assume $M_{1} \leqslant M_{2} \leqslant M_{3}$. We begin by fixing some extensions $u_{M_{i}} \in \overline{F_{\lambda, M_{i}}^{b_{1}}}$ of $u_{i}$ to $\mathbb{R}$ satisfying $\left\|u_{M_{i}}\right\| \frac{}{F_{\lambda, M_{i}}^{b_{1}}} \leqslant 2\left\|u_{i}\right\| \frac{}{F_{\lambda, M_{i}}^{b_{1}}(T)}$.

Let $\gamma: \mathbb{R} \rightarrow[0 ; 1]$ be a smooth partition of unity as in the proof of lemma 6.1 , satisfying now supp $\gamma \subset[-1 ; 1]$ and

$$
\forall t \in \mathbb{R}, \sum_{n \in \mathbb{Z}} \gamma^{3}(t-n)=1
$$

We then use $\gamma$ to chop the time interval in pieces of size $M_{3}^{-1}$ :

$$
\int_{[0 ; T] \times \mathbb{R} \times \mathbb{T}_{\lambda}} u_{1} u_{2} u_{3} \mathrm{~d} t \mathrm{~d} x \mathrm{~d} y \lesssim \sum_{n \in \mathbb{Z}} \int_{\mathbb{R}^{2} \times \lambda^{-1} \mathbb{Z}} f_{1, n} \star f_{2, n} \cdot f_{3, n} \mathrm{~d} \tau \mathrm{d} \xi(\mathrm{d} q)_{\lambda}
$$

where we define

$$
f_{i, n}:=\mathcal{F}\left(\gamma\left(M_{3} t-n\right) \mathbb{1}_{[0, T]} u_{M_{i}}\right)
$$

We can divide the set of integers such that the trilinear form is not zero into two subsets

$$
\begin{array}{r}
\mathcal{A}:=\left\{n \in \mathbb{Z}, \gamma\left(M_{3} t-n\right) \mathbb{1}_{[0, T]}=\gamma\left(M_{3} t-n\right)\right\} \\
\quad \text { and } \mathcal{B}=\left\{n \in \mathbb{Z} \backslash \mathcal{A}, \int f_{1, n} \star f_{2, n} \cdot f_{3, n} \neq 0\right\}
\end{array}
$$

Let us notice that $\# \mathcal{A} \lesssim M_{3}$ and $\# \mathcal{B} \leqslant 4$.

Let us start by dealing with the sum over $\mathcal{A}$ :

$$
\sum_{n \in \mathcal{A}} \int_{\mathbb{R}^{2} \times \lambda^{-1} \mathbb{Z}} f_{1, n} \star f_{2, n} \cdot f_{3, n} \lesssim M_{3} \sup _{n \in \mathcal{A}} \sum_{K_{1}, K_{2}, K_{3} \geqslant M_{3}} \int_{\mathbb{R}^{2} \times \lambda^{-1} \mathbb{Z}} f_{1, n}^{K_{1}} \star f_{2, n}^{K_{2}} \cdot f_{3, n}^{K_{3}}
$$

where $f_{i, n}^{K_{i}}$ is defined as

$$
f_{i, n}^{K_{i}}(\tau, \xi, q):=\rho_{K_{i}}(\tau-\omega(\xi, q)) f_{i, n}(\tau, \xi, q), i=1,2,3 \text { if } K_{i}>M_{3}
$$


and

$$
f_{i, n}^{M_{3}}(\tau, \xi, q):=\chi_{M_{3}}(\tau-\omega(\xi, q)) f_{i, n}(\tau, \xi, q), i=1,2,3
$$

Then, we separate the sum into three parts depending on the relations between the $M$ 's and the $K$ 's as in corollary 5.9 :

$$
\sum_{K_{1}, K_{2}, K_{3}} \int_{\mathbb{R}^{2} \times \lambda^{-1} \mathbb{Z}} f_{1, n}^{K_{1}} \star f_{2, n}^{K_{2}} \cdot f_{3, n}^{K_{3}}=\sum_{i=1}^{3} \sum_{\left(K_{1}, K_{2}, K_{3}\right) \in A_{i}} \int_{\mathbb{R}^{2} \times \lambda^{-1} \mathbb{Z}} f_{1, n}^{K_{1}} \star f_{2, n}^{K_{2}} \cdot f_{3, n}^{K_{3}}
$$

with

$$
\left\{\begin{array}{l}
A_{1}:=\left\{\left(K_{1}, K_{2}, K_{3}\right) \in 2^{\mathbb{N}}, K_{i} \geqslant M_{3}, K_{\max } \leqslant 10^{-10} M_{1} M_{2} M_{3}\right\} \\
A_{2}:=\left\{\left(K_{1}, K_{2}, K_{3}\right) \in 2^{\mathbb{N}}, K_{i} \geqslant M_{3}, K_{1}=K_{\max } \gtrsim M_{1} M_{2} M_{3}\right\} \\
A_{3}:=\left\{\left(K_{1}, K_{2}, K_{3}\right) \in 2^{\mathbb{N}}, K_{i} \geqslant M_{3}, K_{\max }=\left(K_{2} \vee K_{3}\right) \gtrsim M_{1} M_{2} M_{3}\right\}
\end{array}\right.
$$

We treat those terms separately, using the estimates of corollary 5.9. Denoting $J_{i}$ the contributioin of the region $A_{i}$ in the sum, we have

$$
\begin{aligned}
J_{1} \lesssim M_{3} \sup _{n \in \mathcal{A}} \sum_{K_{1}, K_{2}, K_{3} \geqslant M_{3}}\left(M_{\min } \wedge M_{\text {min }}^{-1}\right)^{1 / 2} M_{\text {max }}^{-1} \prod_{i=1}^{3} K_{i}^{1 / 2}\left\|f_{i, n}^{K_{i}}\right\|_{L^{2}} \\
\lesssim\left(M_{\min } \wedge M_{\text {min }}^{-1}\right)^{1 / 2} \prod_{i=1}^{3}\left\|u_{M_{i}}\right\|_{\overline{F_{\lambda, M_{i}}^{0}}}
\end{aligned}
$$

after using (5.27) and

$$
\sup _{n \in \mathcal{A}} \sum_{K_{i} \geqslant M_{3}} K_{i}^{1 / 2}\left\|f_{i, n}^{K_{i}}\right\|_{L^{2}} \lesssim\left\|u_{M_{i}}\right\|_{\overline{F_{\lambda, M_{i}}^{0}}}
$$

Indeed, (7.6) follows from the definition of $f_{i, n}^{K_{i}}(7.5)$, the fact that $\chi_{\left(1 \vee M_{i}\right)^{-1}} \equiv 1$ on the support of $\gamma_{M_{3}^{-1}}$, and the use of (3.10) and (3.11).

Proceeding analogously, we get

$$
\begin{aligned}
J_{3} \lesssim M_{3} \sup _{n \in \mathcal{A}} \sum_{K_{1}, K_{2}, K_{3} \geqslant M_{3}} M_{\max }^{-1}\left(\frac{\left(1 \vee M_{\min }\right)}{M_{\max }}\right)^{1 / 4} \prod_{i=1}^{3} K_{i}^{1 / 2}\left\|f_{i, n}^{K_{i}}\right\|_{L^{2}} \\
\qquad\left(\frac{\left(1 \vee M_{\min }\right)}{M_{\max }}\right)^{2 b_{1}} \prod_{i=1}^{3}\left\|u_{i}\right\| \overline{F_{\lambda, M_{i}}^{0}}
\end{aligned}
$$

by using (5.29) and that $b_{1} \in[0 ; 1 / 8]$.

Finally, the last contribution is controled thanks to (5.28), (7.6) and the weight $\beta_{M_{1}, K_{1}}^{b_{1}}$ :

$$
\begin{aligned}
J_{2} \lesssim \sup _{n \in \mathcal{A}} \sum_{K_{1} \gtrsim M_{1} M_{2} M_{3}} \sum_{K_{2}, K_{3} \geqslant M_{3}}\left(1 \wedge M_{\min }\right)^{1 / 4} \prod_{i=1}^{3} K_{i}^{1 / 2}\left\|f_{i, n}^{K_{i}}\right\|_{L^{2}} \\
\lesssim\left(1 \wedge M_{\min }\right)^{1 / 4}\left(\frac{\left(1 \vee M_{\min }\right)^{3}}{M_{\min } M_{\max }^{2}}\right)^{b_{1}} \prod_{i=1}^{2}\left\|u_{M_{i}}\right\|_{\overline{F_{\lambda, M_{i}}^{0}}} \\
\cdot\left(\sup _{n \in \mathcal{A}} \sum_{K_{1} \gtrsim M_{1} M_{2} M_{3}} \beta_{M_{1}, K_{1}}^{b_{1}} K_{1}^{1 / 2}\left\|f_{1, n}^{K_{1}}\right\|_{L^{2}}\right)
\end{aligned}
$$


This suffices for (7.1) since

$$
\sup _{n \in \mathcal{A}} \sum_{K_{1} \gtrsim M_{1} M_{2} M_{3}} \beta_{M_{1}, K_{1}}^{b_{1}} K_{1}^{1 / 2}\left\|f_{1, n}^{K_{1}}\right\|_{L^{2}} \lesssim\left\|u_{M_{1}}\right\| \overline{F_{\lambda, M_{1}}^{b_{1}}}
$$

as we only need to use (3.11) in this regime.

Let us now come back to (7.4). It remains to treat the border terms. We have

$$
\sum_{n \in \mathcal{B}} \int_{\mathbb{R}^{2} \times \lambda^{-1} \mathbb{Z}} f_{1, n} \star f_{2, n} \cdot f_{3, n} \leqslant \sum_{n \in \mathcal{B}} \sum_{K_{1}, K_{2}, K_{3}} \int_{\mathbb{R}^{2} \times \lambda^{-1} \mathbb{Z}} g_{1, n}^{K_{1}} \star g_{2, n}^{K_{2}} \cdot g_{3, n}^{K_{3}}
$$

where $g_{i, n}^{K_{i}}$ is defined as

$$
g_{i, n}^{K_{i}}:=\rho_{K_{i}}(\tau-\omega) \mathcal{F}\left(\gamma\left(M_{3} t-n\right) \mathbb{1}_{[0, T]} u_{M_{i}}\right), i=1,2,3, K_{i} \geqslant 1
$$

Once again, we separate the different cases of corollary 5.9. Let us define $G_{i}$ the contribution of the region $A_{i}$ in the sum above.

Using (5.27), we can control the first term :

$$
G_{1} \lesssim\left(M_{\min } \wedge M_{\min }^{-1}\right)^{1 / 2} M_{\max }^{-1} \sup _{n \in \mathcal{B}} \sum_{\left(K_{1}, K_{2}, K_{3}\right) \in A_{1}} \prod_{i=1}^{3} K_{i}^{1 / 2}\left\|g_{i, n}^{K_{i}}\right\|_{L^{2}}
$$

Now, we need to replace $(7.6)$ by an analogous estimate on $\mathcal{B}$ :

$$
\sup _{n \in \mathcal{B}} \sup _{K_{i} \geqslant M_{3}} K_{i}^{1 / 2}\left\|\mid g_{i, n}^{K_{i}}\right\|_{L^{2}} \lesssim\left\|u_{M_{i}}\right\| \overline{F_{\lambda, M_{i}}^{0}}
$$

Let us prove this estimate. Using the definition of $g_{i, n}^{K_{i}}(7.7)$, if we note $\widetilde{u_{M_{i}}}:=\gamma\left(M_{3} t-n\right) u_{M_{i}}$ then we have to estimate

$$
\left\|g_{i, n}^{K_{i}}\right\|_{L^{2}}=\left\|\rho_{K_{i}}(\tau-\omega) \cdot \widehat{\mathbb{1}_{[0, T]}} \star \mathcal{F}\left(\widetilde{u_{M_{i}}}\right)\right\|_{L^{2}}
$$

We then split $\mathcal{F}\left(\widetilde{u_{M_{i}}}\right)$ depending on its modulations :

$$
\begin{aligned}
& \left\|g_{i, n}^{K_{i}}\right\|_{L^{2}} \leqslant \sum_{K \leqslant K_{i} / 10}\left\|\rho_{K_{i}}(\tau-\omega) \cdot \widehat{\mathbb{1}_{[0, T]}} \star_{\tau}\left(\rho_{K}\left(\tau^{\prime}-\omega\right) \mathcal{F}\left(\widetilde{u_{M_{i}}}\right)\right)\right\|_{L^{2}} \\
& +\sum_{K \geqslant K_{i} / 10}\left\|\rho_{K_{i}}(\tau-\omega) \mathcal{F}_{t}\left\{\mathbb{1}_{[0, T]} \mathcal{F}_{t}^{-1}\left(\rho_{K}\left(\tau^{\prime}-\omega\right) \mathcal{F}\left(\widetilde{u_{M_{i}}}\right)\right)\right\}\right\|_{L^{2}}
\end{aligned}
$$$$
=I+I I
$$

To treat $I$, we use that $\left|\widehat{\mathbb{1}_{[0, T]}}\left(\tau-\tau^{\prime}\right)\right| \leqslant\left|\tau-\tau^{\prime}\right|^{-1} \sim K_{i}^{-1}$ since $|\tau-\omega| \sim K_{i}$ and $\left|\tau^{\prime}-\omega\right| \sim$ $K \leqslant K_{i} / 10$. Thus, from Young inequality $L^{\infty} \times L^{1} \rightarrow L^{\infty}$ we deduce that

$$
K_{i}^{1 / 2} \cdot I \lesssim K_{i}\left\|\left.\widehat{\mathbb{1}_{[0, T]} \star_{\tau}}\left(\rho_{K}\left(\tau^{\prime}-\omega\right) \mathcal{F}\left(\widetilde{u_{M_{i}}}\right)\right)\right|_{L_{\xi, q}^{2} L_{\tau}^{\infty}} \lesssim\right\| \rho_{K}\left(\tau^{\prime}-\omega\right) \mathcal{F}\left(\widetilde{u_{M_{i}}}\right) \|_{L_{\xi, q}^{2} L_{\tau}^{1}}
$$

which is enough for (7.9) due to (3.12) and then (3.10)-(3.11). 
To deal with $I I$, we simply neglect the localization $\rho_{K_{i}}(\tau-\omega)$, use Plancherel identity, then neglect the localization $\mathbb{1}_{[0 ; T]}$ and use Plancherel identity again and that $K_{i}^{1 / 2} \lesssim K^{1 / 2}$ to get

$$
K_{i}^{1 / 2} \cdot I I \lesssim \sum_{K \geqslant K_{i} / 10} K^{1 / 2}\left\|\left.\rho_{K}\left(\tau^{\prime}-\omega\right) \mathcal{F}\left(\widetilde{u_{M_{i}}}\right)\right|_{L_{\xi, q, \tau}^{2}} \lesssim\right\| \mathcal{F}\left(\widetilde{u_{M_{i}}}\right) \|_{X_{\lambda, M_{i}}^{0}}
$$

This proves (7.9) after using again (3.10)-(3.11).

Coming back to (7.8) and using (7.9) along with $\# \mathcal{B} \leqslant 4$, we then infer

$$
G_{1} \lesssim\left\langle\ln \left(M_{1} M_{2} M_{3}\right)\right\rangle^{3}\left(M_{\min } \wedge M_{\min }^{-1}\right)^{1 / 2} M_{\max }^{-1} \prod_{i=1}^{3}\left\|u_{i}\right\| \overline{F_{\lambda, M_{i}}^{0}}
$$

as $\sum_{\left(K_{1}, K_{2}, K_{3}\right) \in A_{1}} 1 \lesssim\left\langle\ln \left(M_{1} M_{2}\right)\right\rangle^{3}$. This is enough for (7.1).

Let us now turn to $G_{2}$. We use (5.28) combined with (7.9) to get

$$
\begin{array}{r}
G_{2} \lesssim\left(1 \wedge M_{\min }\right)^{1 / 4} M_{\min }^{0+} M_{\max }^{(-1)+} \sum_{\left(K_{1}, K_{2}, K_{3}\right) \in A_{2}} K_{\max }^{0-} \prod_{i=1}^{3} \sup _{n \in \mathcal{B} K_{i}} K_{i}^{1 / 2}\left\|g_{i, n}^{K_{i}}\right\|_{L^{2}} \\
\lesssim M_{\max }^{(-1)+} \prod_{i=1}^{3}\left\|u_{M_{i}}\right\| \frac{}{F_{\lambda, M_{i}}^{0}}
\end{array}
$$

which is sufficient as well.

Finally, we treat $G_{3}$, using now (5.29) and (7.9) :

$$
\begin{aligned}
G_{3} \lesssim\left(1 \vee M_{\min }\right)^{(1 / 4)+} M_{\max }^{(-5 / 4)+} \sum_{\left(K_{1}, K_{2}, K_{3}\right) \in A_{3}} K_{\max }^{0-} \prod_{i=1}^{3} \sup _{n \in \mathcal{B} K_{i}}\left\|g_{i, n}^{K_{i}}\right\|_{L^{2}} \\
\lesssim M_{\max }^{(-1)+} \prod_{i=1}^{3}\left\|u_{M_{i}}\right\|_{\overline{F_{\lambda, M_{i}}^{0}}}
\end{aligned}
$$

which concludes the proof of lemma 7.1.

Following [9, Lemme 6.1 (b)], we then use the previous estimate to control the special terms in the energy estimate 1.13 :

\section{Lemma 7.2}

$$
\begin{aligned}
& \text { Let } T \in] 0 ; 1], b_{1} \in[0 ; 1 / 8], M, M_{1} \in 2^{\mathbb{Z}} \text {, with } M \geqslant 10\left(1 \vee M_{1}\right) \text {, and } u \in \overline{F_{\lambda, M}^{b_{1}}}(T) \text {, } \\
& v \in \overline{F_{\lambda, M_{1}}^{b_{1}}}(T) \text {. Then } \\
& \left|\int_{[0, T] \times \mathbb{R} \times \mathbb{T}_{\lambda}} P_{M} u \cdot P_{M}\left(P_{M_{1}} v \cdot \partial_{x} u\right) \mathrm{d} t \mathrm{~d} x \mathrm{~d} y\right| \\
& \lesssim M_{1} \Lambda_{b_{1}}\left(M_{1}, M\right)\left\|P_{M_{1}} v\right\| \overline{F_{\lambda, M_{1}}^{b_{1}}(T)} \sum_{M_{2} \sim M}\left\|P_{M_{2}} u\right\|_{F_{\lambda, M_{2}}^{b_{1}}(T)}^{2}
\end{aligned}
$$


Proof :

First, we chop the integral in the left-hand side of (7.10) into two terms

$$
\begin{aligned}
& \int_{[0, T] \times \mathbb{R} \times \mathbb{\mathbb { T }}_{\lambda}} P_{M} u \cdot P_{M}\left(\partial_{x} u P_{M_{1}} v\right) \\
&= \int_{[0, T] \times \mathbb{R} \times \mathbb{\mathbb { N }}_{\lambda}} P_{M} u \cdot P_{M} \partial_{x} u \cdot P_{M_{1}} v+\int_{[0, T] \times \mathbb{R} \times \mathbb{\mathbb { N }}_{\lambda}} P_{M} u \cdot\left[P_{M}\left(\partial_{x} u P_{M_{1}} v\right)-P_{M} \partial_{x} u \cdot P_{M_{1}} v\right] \\
&=I+I I
\end{aligned}
$$

The first term is easy to control : integrating by parts and using (7.1), we get the bound

$$
\begin{aligned}
|I|=\left|\frac{1}{2} \int_{[0, T] \times \mathbb{R} \times \mathbb{T}_{\lambda}}\left(P_{M} u\right)^{2} \cdot \partial_{x} P_{M_{1}} v\right| & \lesssim M_{1} \Lambda_{b_{1}}\left(M_{1}, M\right)\left\|P_{M} u\right\| \frac{2}{F_{\lambda, M}^{b_{1}}(T)}\left\|P_{M_{1}} v\right\| \frac{}{F_{\lambda, M_{1}}^{b_{1}}(T)}
\end{aligned}
$$

To deal with $I I$, we proceed as for the previous lemma : after choosing some extensions (still denoted $u \in \overline{F_{\lambda, M}^{b_{1}}}$ and $v \in \overline{F_{\lambda, M_{1}}^{b_{1}}}$ ) of $u$ and $v$ to $\mathbb{R}$, we chop the integral in

$$
I I=\sum_{n \in \mathbb{Z}} \int_{\mathbb{R}^{2} \times \mathbb{T}_{\lambda}} P_{M} u_{n} \cdot\left[P_{M}\left(\partial_{x} u_{n} P_{M_{1}} v_{n}\right)-P_{M} \partial_{x} u_{n} \cdot P_{M_{1}} v_{n}\right]
$$

where we define $u_{n}:=\mathbb{1}_{[0, T]} \gamma(M t-n) u$ and $v_{n}:=\mathbb{1}_{[0, T]} \gamma(M t-n) v$ for a function $\gamma$ as in the previous lemma.

Using Plancherel identity, we can write $I I$ as

$$
I I=\sum_{n \in \mathbb{Z}} \int_{\mathbb{R}^{2} \times \lambda^{-1} \mathbb{Z}} \widehat{P_{M} u_{n}} \cdot \int_{\mathbb{R}^{2} \times \lambda^{-1} \mathbb{Z}} K\left(\zeta, \zeta_{1}\right) \widehat{u_{n}}\left(\zeta-\zeta_{1}\right) \partial_{x} \widehat{P_{M_{1}} v_{n}}\left(\zeta_{1}\right) \mathrm{d} \zeta_{1} \mathrm{~d} \zeta
$$

where the kernel $K$ is given by

$$
K\left(\zeta, \zeta_{1}\right)=\frac{\xi-\xi_{1}}{\xi_{1}}\left[\eta_{M}(\xi)-\eta_{M}\left(\xi-\xi_{1}\right)\right] \widetilde{\eta_{M_{1}}}\left(\xi_{1}\right) \sum_{M_{2} \sim M} \eta_{M_{2}}\left(\xi-\xi_{1}\right)
$$

The last sum appears since $|\xi| \sim M$ and $\left|\xi_{1}\right| \sim M_{1} \leqslant M / 10$, thus $\left|\xi-\xi_{1}\right| \sim M$. Using the mean value theorem, we can bound the kernel with

$$
\left|K\left(\zeta, \zeta_{1}\right)\right| \lesssim\left|\frac{\xi-\xi_{1}}{\xi_{1}}\right| M^{-1}\left|\xi_{1}\right| \widetilde{\eta_{M_{1}}}\left(\xi_{1}\right) \sum_{M_{2} \sim M} \eta_{M_{2}}\left(\xi-\xi_{1}\right) \lesssim \widetilde{\eta_{M_{1}}}\left(\xi_{1}\right) \sum_{M_{2} \sim M} \eta_{M_{2}}\left(\xi-\xi_{1}\right)
$$

Therefore, as in [9, Lemma 6.1 (b)], (7.10) follows after repeating the proof of (7.1) and using (7.11).

We finally prove (1.13). From now on, we fix $b_{1}=1 / 8$ and drop the parameter when writing the main spaces. 


\section{Proposition 7.3}

Let $T \in] 0 ; 1]$ and $u \in \mathcal{C}\left([-T, T], \mathbf{E}_{\lambda}^{\infty}\right)$ be a solution of

$$
\left\{\begin{array}{l}
\partial_{t} u+\partial_{x}^{3} u-\partial_{x}^{-1} \partial_{y} u+u \partial_{x} u=0 \\
u(0, x)=u_{0}(x)
\end{array}\right.
$$

on $[-T, T]$. Then for any $\alpha \geqslant 1$,

$$
\|u\|_{\mathbf{B}_{\lambda}^{\alpha}(T)}^{2} \lesssim\left\|u_{0}\right\|_{\mathbf{E}_{\lambda}^{\alpha}}^{2}+\|u\|_{\mathbf{F}_{\lambda}(T)}\|u\|_{\mathbf{F}_{\lambda}^{\alpha}(T)}^{2}
$$

Proof :

Using the definitions of $\mathbf{B}_{\lambda}^{\alpha}(T)$ (3.4) and $p$ (2.1) along with (3.5), it suffices to prove

$$
\begin{aligned}
\sum_{M_{3} \geqslant 1} \sup _{t_{M_{3}} \in[-T ; T]} M_{3}^{2 \alpha}\left\|P_{M_{3}} u\left(t_{M_{3}}\right)\right\|_{L^{2}}^{2}- & M_{3}^{2 \alpha}\left\|P_{M_{3}} u_{0}\right\|_{L^{2}}^{2} \\
& \lesssim\|u\|_{\mathbf{F}_{\lambda}(T)} \sum_{M_{3} \geqslant 1} M_{3}^{2 \alpha}\left\|P_{M_{3}} u\right\|_{F_{\lambda, M_{3}}^{b_{1}}(T)}^{2}
\end{aligned}
$$

and

$$
\begin{aligned}
\sum_{M_{3} \geqslant 1} \sup _{t_{M_{3}} \in[-T ; T]} M_{3}^{2(\alpha-1)}\left\|P_{M_{3}} \partial_{x}^{-1} \partial_{y} u\left(t_{M_{3}}\right)\right\|_{L^{2}}^{2}-M_{3}^{2(\alpha-1)}\left\|P_{M_{3}} \partial_{x}^{-1} \partial_{y} u_{0}\right\|_{L^{2}}^{2} \\
\lesssim\|u\|_{\mathbf{F}_{\lambda}(T)} \sum_{M_{3} \geqslant 1} M_{3}^{2 \alpha}\left\|P_{M_{3}} u\right\|_{F_{\lambda, M_{3}}^{b_{1}}(T)}^{2}
\end{aligned}
$$

Let us start with (7.14).

Applying $P_{M_{3}}$ to (7.12), multiplying by $P_{M_{3}} u$ and integrating, we get

$$
\begin{aligned}
\left\|P_{M_{3}} u\left(t_{M_{3}}\right)\right\|_{L^{2}}^{2}-\left\|P_{M_{3}} u_{0}\right\|_{L^{2}}^{2}=\int_{0}^{t_{M_{3}}} & \frac{\mathrm{d}}{\mathrm{d} t}\left\|P_{M_{3}} u(t)\right\|_{L^{2}}^{2} \mathrm{~d} t \\
& \lesssim\left|\int_{0}^{t_{M_{3}}} \int_{\mathbb{R} \times \mathbb{T}_{\lambda}} P_{M_{3}} u \cdot P_{M_{3}}\left(u \partial_{x} u\right) \mathrm{d} t^{\prime} \mathrm{d} x \mathrm{~d} y\right|
\end{aligned}
$$

since $\partial_{x}^{3}$ and $\partial_{x}^{-1} \partial_{y}^{2}$ are skew-adjoint.

We separate the right-hand side of (7.16) in

$$
\begin{aligned}
& \sum_{M_{1} \leqslant M_{3} / 10} \int_{\left[0, t_{M_{3}}\right] \times \mathbb{R} \times \mathbb{T}_{\lambda}} P_{M_{3}} u \cdot P_{M_{3}}\left(P_{M_{1}} u \cdot \partial_{x} u\right) \mathrm{d} t \mathrm{~d} x \mathrm{~d} y \\
& +\sum_{M_{1} \gtrsim M_{3}} \sum_{M_{2}>0} \int_{\left[0, t_{M_{3}}\right] \times \mathbb{R} \times \mathbb{T}_{\lambda}}\left(P_{M_{3}}\right)^{2} u \cdot P_{M_{1}} u \cdot \partial_{x} P_{M_{2}} u \mathrm{~d} t \mathrm{~d} x \mathrm{~d} y
\end{aligned}
$$

Using (7.10) and Cauchy-Schwarz inequality in $M_{1}$, the first term (7.17) is estimated by

$$
\begin{aligned}
(7.17) \lesssim \sum_{M_{1} \leqslant M_{3} / 10} M_{1} \Lambda_{b_{1}}\left(M_{1}, M_{3}\right)\left\|P_{M_{1}} u\right\|_{F_{\lambda, M_{1}}^{b_{1}}}(T) & \sum_{M_{2} \sim M_{3}}\left\|P_{M_{2}} u\right\| \frac{2}{F_{\lambda, M_{2}}^{b_{1}}(T)} \\
& \lesssim\|u\|_{\mathbf{F}_{\lambda}(T)} \sum_{M_{2} \sim M}\left\|P_{M_{2}} u\right\|_{F_{\lambda, M_{2}}^{b_{1}}(T)}^{2}
\end{aligned}
$$


since

$$
\left[\sum_{0<M_{1} \leqslant M_{3} / 10}\left(1 \vee M_{1}\right)^{-2} M_{1}^{2} \Lambda_{b_{1}}\left(M_{1}, M_{3}\right)^{2}\right]^{1 / 2} \lesssim 1
$$

Thus

$$
\sum_{M_{3} \geqslant 1} M_{3}^{2 \alpha} \cdot(7.17) \lesssim\|u\|_{\mathbf{F}_{\lambda}(T)}\|u\|_{\mathbf{F}_{\lambda}^{\alpha}(T)}^{2}
$$

To treat (7.18), we use (7.1) and then we separate the sum on $M_{2}$ depending on whether $M_{1} \sim M_{3} \gtrsim M_{2}$ or $M_{1} \sim M_{2} \gtrsim M_{3}$ :

$$
\begin{aligned}
(7.18) \lesssim \sum_{M_{1} \sim M_{3}} & \sum_{M_{2} \lesssim M_{3}} M_{2} \Lambda_{b_{1}}\left(M_{2}, M_{3}\right) \prod_{i=1}^{3}\left\|P_{M_{i}} u\right\|_{\overline{F_{\lambda, M_{i}}^{b_{1}}}(T)} \\
& +\sum_{M_{1} \gtrsim M_{3}} \sum_{M_{2} \sim M_{1}} M_{2} \Lambda_{b_{1}}\left(M_{3}, M_{2}\right) \prod_{i=1}^{3}\left\|P_{M_{i}} u\right\| \overline{F_{\lambda, M_{i}}^{b_{1}}}(T)
\end{aligned}
$$$$
=I+I I
$$

Applying Cauchy-Schwarz inequality in $M_{2}$ we get the bounds

$$
I \lesssim\left\|P_{M_{3}} u\right\| \frac{2}{F_{\lambda, M_{3}}^{b_{1}}(T)}\|u\|_{\mathbf{F}_{\lambda}(T)}
$$

and

$$
I I \lesssim \sum_{M_{1} \gtrsim M_{3}} M_{1} \Lambda_{b_{1}}\left(M_{3}, M_{1}\right)\left\|P_{M_{3}} u\right\| \frac{}{F_{\lambda, M_{3}}^{b_{1}}(T)}\left\|P_{M_{1}} u\right\| \|_{F_{\lambda, M_{1}}^{b_{1}}(T)}^{2}
$$

Summing on $M_{3}$ and using Cauchy-Schwarz inequality in $M_{3}$ and $M_{1}$ for $I I$, we finally get

$$
\begin{aligned}
& \sum_{M_{3} \geqslant 1} M_{3}^{2 \alpha} \cdot(7.18) \lesssim\|u\|_{\mathbf{F}_{\lambda}(T)}\|u\|_{\mathbf{F}_{\lambda}^{\alpha}(T)}^{2} \\
&+\left.\sum_{M_{3} \geqslant 1} \sum_{M_{1} \gtrsim M_{3}} M_{3}^{\alpha} \Lambda_{b_{1}}\left(M_{3}, M_{1}\right) M_{1}^{1+\alpha}\left\|P_{M_{3}} u\right\|{\overline{F_{\lambda, M_{3}}^{b_{1}}(T)}}\left\|P_{M_{1}} u\right\|\right|_{F_{\lambda, M_{1}}^{b_{1}}(T)} ^{2} \\
& \quad \lesssim\|u\|_{\mathbf{F}_{\lambda}(T)}\|u\|_{\mathbf{F}_{\lambda}^{\alpha}(T)}^{2}
\end{aligned}
$$

Now we turn to the proof of (7.15).

This time, we apply $P_{M_{3}} \partial_{x}^{-1} \partial_{y}$ to (1.6), we multiply by $P_{M_{3}} \partial_{x}^{-1} \partial_{y} u$ and we integrate to get

$$
\begin{aligned}
\|\left. P_{M_{3}} \partial_{x}^{-1} \partial_{y} u\left(t_{M_{3}}\right)\right|_{L^{2}} ^{2}- & \left\|P_{M_{3}} \partial_{x}^{-1} \partial_{y} u_{0}\right\|_{L^{2}}^{2} \\
& \lesssim\left|\int_{\left[0, t_{M_{3}}\right] \times \mathbb{R} \times \mathbb{V}_{\lambda}} P_{M_{3}} \partial_{x}^{-1} \partial_{y} u \cdot P_{M_{3}} \partial_{x}^{-1} \partial_{y}\left(u \partial_{x} u\right) \mathrm{d} t \mathrm{~d} x \mathrm{~d} y\right|
\end{aligned}
$$

using again the skew-adjointness of $\partial_{x}^{3}$ and $\partial_{x}^{-1} \partial_{y}^{2}$.

The right-hand side of (7.19) is similarly split up into

$$
\begin{aligned}
& \sum_{M_{1} \leqslant M_{3} / 10} \int_{\left[0, t_{M_{3}}\right] \times \mathbb{R} \times \mathbb{T}_{\lambda}} P_{M_{3}} \partial_{x}^{-1} \partial_{y} u \cdot P_{M_{3}}\left(P_{M_{1}} u \cdot \partial_{y} u\right) \mathrm{d} t \mathrm{~d} x \mathrm{~d} y \\
+ & \sum_{M_{1} \gtrsim M_{3}} \sum_{M_{2}} \int_{\left[0, t_{M_{3}}\right] \times \mathbb{R} \times \mathbb{T}_{\lambda}}\left(P_{M_{3}}\right)^{2} \partial_{x}^{-1} \partial_{y} u \cdot P_{M_{1}} u \cdot \partial_{y} P_{M_{2}} u \mathrm{~d} t \mathrm{~d} x \mathrm{~d} y
\end{aligned}
$$


Writing $v:=\partial_{x}^{-1} \partial_{y} u$, using (7.10) and Cauchy-Schwarz inequality in $M_{1}$, we obtain

$$
\begin{aligned}
(7.20) \lesssim \sum_{M_{1} \leqslant M_{3} / 10} M_{1} \Lambda_{b_{1}}\left(M_{1}, M_{3}\right)\left\|P_{M_{1}} u\right\| \frac{\sum_{F_{\lambda, M_{1}}^{b_{1}}}(T)}{} \sum_{M_{2} \sim M_{3}}\left\|v_{M_{2}}\right\| \|_{F_{\lambda, M_{2}}^{b_{1}}(T)}^{2} \\
\\
\lesssim\|u\|_{\mathbf{F}_{\lambda}(T)} \sum_{M_{2} \sim M_{3}}\left\|P_{M_{2}} \partial_{x}^{-1} \partial_{y} u\right\| \frac{2}{F_{\lambda, M_{2}}^{b_{1}}(T)}
\end{aligned}
$$

which is enough for (7.15) after summing on $M_{3}$.

As for (7.21), we separate again the sum on $M_{2}$ :

$$
\begin{aligned}
(7.21)=I+I I=\sum_{M_{1} \sim M_{3}} & \sum_{M_{2} \lesssim M_{3}} \int_{\left[0, t_{M_{3}}\right] \times \mathbb{R} \times \mathbb{T}_{\lambda}}\left(P_{M_{3}}\right)^{2} v \cdot P_{M_{1}} u \cdot \partial_{x} v_{M_{2}} \mathrm{~d} t \mathrm{~d} x \mathrm{~d} y \\
& \quad+\sum_{M_{1} \gtrsim M_{3}} \sum_{M_{2} \sim M_{1}} \int_{\left[0, t_{M_{3}}\right] \times \mathbb{R} \times \mathbb{T}_{\lambda}}\left(P_{M_{3}}\right)^{2} v \cdot P_{M_{1}} u \cdot \partial_{x} P_{M_{2}} v \mathrm{~d} t \mathrm{~d} x \mathrm{~d} y
\end{aligned}
$$

For the first term, we use again (7.1) which gives

$$
I \lesssim \sum_{M_{1} \sim M_{3}} \sum_{M_{2} \lesssim M_{3}} M_{2} \Lambda_{b_{1}}\left(M_{2}, M_{3}\right)\left\|P_{M_{3}} v\right\|_{\overline{F_{\lambda, M_{3}}^{b_{1}}}(T)}\left\|P_{M_{1}} u\right\|_{\overline{F_{\lambda, M_{1}}^{b_{1}}(T)}}\left\|P_{M_{2}} v\right\|_{\overline{F_{\lambda, M_{2}}^{b_{1}}(T)}}
$$

We first sum on $M_{2}$ by using Cauchy-Schwarz inequality to get the bound

$$
\sum_{M_{1} \sim M_{3}} M_{3}\left\|P_{M_{3}} v\right\|_{\overline{F_{\lambda, M_{3}}^{b_{1}}}(T)}\left\|P_{M_{1}} u\right\|_{\overline{F_{\lambda, M_{1}}^{b_{1}}(T)}}\|u\|_{\mathbf{F}_{\lambda}(T)}
$$

and then we can sum on $M_{3}$ using Cauchy-Schwarz inequality again to get (7.15) for this term.

For the second term, we apply also (7.1), then we first sum on $M_{3}$ using Cauchy-Schwarz inequality and $M_{3}^{2(\alpha-1)} \lesssim M_{1}^{2(\alpha-1)}$ in this regime, and finally sum on $M_{1}$ using again Cauchy-Schwarz inequality to get (7.15).

In the same spirit, following [9] we have for the difference equation

\section{Proposition 7.4}

Let $T \in] 0,1\left[\right.$ and $u, v \in \mathbf{F}_{\lambda}(T)$ satisfying

$$
\left\{\begin{array}{l}
\partial_{t} u+\partial_{x}^{3} u-\partial_{x}^{-1} \partial_{y} u+\partial_{x}(u v)=0 \\
u(0, x)=u_{0}(x)
\end{array}\right.
$$

on $[-T, T] \times \mathbb{R} \times \mathbb{T}_{\lambda}$. Then

$$
\|u\|_{\overline{\mathbf{B}_{\lambda}(T)}}^{2} \lesssim\left\|u_{0}\right\|_{L_{\lambda}^{2}}^{2}+\left\|\left.v\right|_{\mathbf{F}_{\lambda}(T)}\right\| u \|_{\overline{\mathbf{F}_{\lambda}(T)}}^{2}
$$

and to deal with the equation satisfied by $P_{H i g h} \partial_{x}\left(u_{1}-u_{2}\right)$ we need 


\section{Proposition 7.5}

Let $T \in] 0 ; 1]$ and $u \in \overline{\mathbf{F}_{\lambda}}(T)$ with $u=P_{\text {High }} u$. Moreover, let $v \in \mathbf{F}_{\lambda}(T), w_{i} \in \mathbf{F}_{\lambda}(T)$, $i=1,2,3$, and $w_{i}^{\prime} \in \overline{\mathbf{F}_{\lambda}}(T), i=1,2,3$ and finally $h \in \overline{\mathbf{F}_{\lambda}}(T)$ with $h=P_{\leqslant 1} h$. Assume that $u$ satisfies

$$
\partial_{t} u+\partial_{x}^{3} u-\partial_{x}^{-1} \partial_{y}^{2} u=P_{H i g h}\left(v \partial_{x} u\right)+\sum_{i=1}^{3} P_{H i g h}\left(w_{i} w_{i}^{\prime}\right)+P_{H i g h} h
$$

on $[-T ; T] \times \mathbb{R} \times \mathbb{T}_{\lambda}$. Then

$$
\|u\|_{\overline{\mathbf{B}_{\lambda}}(T)}^{2} \lesssim\left\|u_{0}\right\|_{L_{\lambda}^{2}}^{2}+\left.\left\|\left.v\right|_{\mathbf{F}_{\lambda}(T)}\right\| u\left\|_{\mathbf{F}_{\lambda}(T)}^{2}+\right\| u\right|_{\overline{\mathbf{F}_{\lambda}}(T)} \sum_{i=1}^{3}\left\|w_{i}\right\|_{\overline{\mathbf{F}_{\lambda}}(T)}\left\|w_{i}^{\prime}\right\|_{\overline{\mathbf{F}_{\lambda}}(T)}
$$

Proof :

(7.23) follows from (7.25) after splitting up $u$ into $P_{\text {Low }} u$ and $P_{H i g h} u$ and observing that $P_{H i g h} u$ satisfies an equation of type (7.24).

To prove (7.25), we follow the proof of proposition 7.3. Using the definitions of $\overline{\mathbf{B}_{\lambda}}(T)$ (3.6), it suffices to prove

$$
\begin{aligned}
\sum_{M_{3}>1} \sup _{M_{3} \in[-T ; T]}\left\|P_{M_{3}} u\left(t_{M_{3}}\right)\right\|_{L^{2}}^{2}-\left\|P_{M_{3}} u_{0}\right\|_{L^{2}}^{2} & \\
& \lesssim\|v\|_{\mathbf{F}_{\lambda}(T)}\|u\|_{\overline{\mathbf{F}_{\lambda}(T)}}^{2}+\|u\|_{\overline{\mathbf{F}_{\lambda}(T)}} \sum_{i=1}^{3}\left\|w_{i}\right\|_{\overline{\mathbf{F}_{\lambda}(T)}}\left\|w_{i}^{\prime}\right\|_{\overline{\mathbf{F}_{\lambda}(T)}}
\end{aligned}
$$

Take $M_{3}>1$. Applying $P_{M_{3}}$ to (7.24), multiplying by $P_{M_{3}} u$ and integrating, we get

$$
\begin{aligned}
\left\|P_{M_{3}} u\left(t_{M_{3}}\right)\right\|_{L^{2}}^{2}- & \left\|P_{M_{3}} u_{0}\right\|_{L^{2}}^{2}=\int_{0}^{t_{M_{3}}} \frac{\mathrm{d}}{\mathrm{d} t}\left\|P_{M_{3}} u(t)\right\|_{L^{2}}^{2} \mathrm{~d} t \\
& \lesssim\left|\int_{0}^{t_{M_{3}}} \int_{\mathbb{R} \times \mathbb{T}_{\lambda}} P_{M_{3}} u \cdot P_{M_{3}} P_{H i g h}\left(u \partial_{x} v\right) \mathrm{d} t^{\prime} \mathrm{d} x \mathrm{~d} y\right| \\
& \quad+\sum_{i=1}^{3}\left|\int_{0}^{t_{M_{3}}} \int_{\mathbb{R} \times \mathbb{T}_{\lambda}} P_{M_{3}} u \cdot P_{M_{3}} P_{H i g h}\left(w_{i} w_{i}^{\prime}\right) \mathrm{d} t^{\prime} \mathrm{d} x \mathrm{~d} y\right|
\end{aligned}
$$

since $\partial_{x}^{3}$ and $\partial_{x}^{-1} \partial_{y}^{2}$ are skew-adjoint. The term in $h$ vanishes after applying $P_{M_{3}}$, due to its frequency localization.

To treat the first term in the right-hand side of (7.27) we split it up in

$$
\begin{aligned}
& \sum_{M_{1} \leqslant M_{3} / 10} \int_{\left[0, t_{M_{3}}\right] \times \mathbb{R} \times \mathbb{T}_{\lambda}} P_{M_{3}} u \cdot P_{M_{3}}\left(P_{M_{1}} v \cdot \partial_{x} u\right) \mathrm{d} t \mathrm{~d} x \mathrm{~d} y \\
& +\sum_{M_{1} \gtrsim M_{3}} \sum_{M_{2}} \int_{\left[0, t_{M_{3}}\right] \times \mathbb{R} \times \mathbb{T}_{\lambda}}\left(P_{M_{3}}\right)^{2} u \cdot P_{M_{1}} v \cdot \partial_{x} P_{M_{2}} u \mathrm{~d} t \mathrm{~d} x \mathrm{~d} y
\end{aligned}
$$


The first term (7.28) is estimated similarly to (7.20) with $\alpha=1$ and exchanging the roles of $u$ and $v$, whereas for $(7.29)$ we proceed as for $(7.21)$.

To treat the second term in the right-hand side of (7.27), we perform a dyadic decomposition of $w_{i}$ and $w_{i}^{\prime}$. By symmetry we can assume $M_{1} \leqslant M_{2}$, thus either $M_{1} \lesssim M_{2} \sim M_{3}$ or $M_{3} \lesssim M_{1} \sim M_{2}$. Then we apply (7.1) to bound the sum on $M_{3}$ by

$$
\begin{aligned}
& \sum_{M_{3} \geqslant 1} \sum_{M_{2} \sim M_{3}} \sum_{M_{1} \lesssim M_{2}} \Lambda_{b_{1}}\left(M_{1}, M_{2}\right)
\end{aligned}
$$

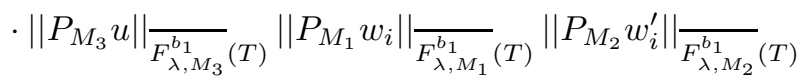

$$
\begin{aligned}
& +\sum_{M_{2} \geqslant 1} \sum_{M_{1} \sim M_{2}} \sum_{1 \leqslant M_{3} \lesssim M_{2}} \Lambda_{b_{1}}\left(M_{3}, M_{2}\right)
\end{aligned}
$$

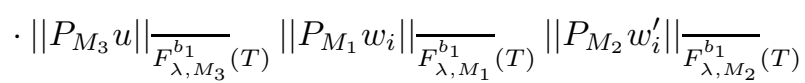

For the second term, we can just use Cauchy-Schwarz inequality in $M_{3}$ and $M_{2}$ since $M_{1} \sim$ $M_{2} \gtrsim M_{3} \geqslant 1$. For the first term, we use that

$$
\sum_{0<M_{1} \lesssim M_{2}} \Lambda_{b_{1}}\left(M_{1}, M_{2}\right)\left\|P_{M_{1}} w_{i}\right\|_{\overline{F_{\lambda, M_{1}}}(T)} \lesssim\left\|w_{i}\right\|_{\overline{\mathbf{F}_{\lambda}}(T)}
$$

Note that this is the only step where we need (5.8) to avoid a logarithmic divergence when summing on very low frequencies, thus we do not need the extra decay for low frequency as in [9].

Thus we finally obtain

$$
\sum_{M \geqslant 1}(7.29) \lesssim \sum_{i=1}^{3}\|u\|_{\overline{\mathbf{F}_{\lambda}}(T)}|| w_{i}\left\|_{\overline{\mathbf{F}_{\lambda}}(T)}\right\| w_{i}^{\prime} \|\left.\right|_{\overline{\mathbf{F}_{\lambda}}(T)}
$$

which concludes the proof of (7.25).

\section{Proof of Theorem 1.1}

We finally turn to the proof of our main result. We follow the scheme of [14, Section 6].

We begin by recalling a local well-posedness result for smooth data :

\section{Proposition 8.1}

Assume $u_{0} \in \mathbf{E}_{\lambda}^{\infty}$. Then there exists $\left.\left.T_{\lambda} \in\right] 0 ; 1\right]$ and a unique solution $u \in \mathcal{C}\left(\left[-T_{\lambda} ; T_{\lambda}\right], \mathbf{E}_{\lambda}^{\infty}\right)$ of (1.6) on $\left[-T_{\lambda} ; T_{\lambda}\right] \times \mathbb{R} \times \mathbb{T}_{\lambda}$. Moreover, $T_{\lambda}=T\left(\left\|u_{0}\right\|_{\mathbf{E}_{\lambda}^{3}}\right)$ can be chosen as a nonincreasing function of $\left\|u_{0}\right\|_{\mathbf{E}_{\lambda}^{3}}$.

\section{Proof :}

This is a straightforward adaptation of [10] to the case of partially periodic data. Indeed, proposition 8.1 follows from the standard energy estimate (see for example [13, Lemma 1.3])

$$
\left\|\left.u\right|_{L_{T}^{\infty} \mathbf{E}_{\lambda}^{\alpha}} \leqslant C_{\alpha}\right\| u_{0} \|_{\mathbf{E}_{\lambda}^{\alpha}} \exp \left(\widetilde{C}_{\alpha}\left\|\partial_{x} u\right\|_{L_{T}^{1} L_{x y}^{\infty}}\right)
$$


along with the Sobolev embedding

$$
\left\|\partial_{x} u\right\|_{L_{T}^{1} L_{x y}^{\infty}} \lesssim T\|u\|_{L_{T}^{\infty} \mathbf{E}_{\lambda}^{3}}
$$

\subsection{A priori estimates for smooth solutions}

In this subsection we improve the control on the previous solutions.

\section{Proposition 8.2}

There exists $\left.\left.\epsilon_{0} \in\right] 0 ; 1\right]$ such that for $u_{0} \in \mathbf{E}_{\lambda}^{\infty}$ with

$$
\left\|u_{0}\right\|_{\mathbf{E}_{\lambda}} \leqslant \epsilon_{0}
$$

then there exists a unique solution $u$ to $(1.6)$ in $\mathcal{C}\left([-1 ; 1], \mathbf{E}_{\lambda}^{\infty}\right)$, and it satisfies for $\alpha=$ $1,2,3$,

$$
\|u\|_{\mathbf{F}_{\lambda}^{\alpha}(1)} \leqslant C_{\alpha}\left\|u_{0}\right\|_{\mathbf{E}_{\lambda}^{\alpha}}
$$

where $C_{\alpha}>0$ is a constant independent of $\lambda$.

\section{Proof :}

Let $\left.\left.T=T\left(\left\|u_{0}\right\|_{\mathbf{E}_{\lambda}^{3}}\right) \in\right] 0 ; 1\right]$ and $u \in \mathcal{C}\left([-T ; T], \mathbf{E}_{\lambda}^{\infty}\right)$ be the solution to (1.6) given by proposition 8.1. Then, for $T^{\prime} \in[0 ; T]$, we define

$$
\mathcal{X}_{\lambda, \alpha}\left(T^{\prime}\right):=\|u\|_{\mathbf{B}_{\lambda}^{\alpha}\left(T^{\prime}\right)}+\left\|u \partial_{x} u\right\|_{\mathbf{N}_{\lambda}^{\alpha}\left(T^{\prime}\right)}
$$

Recalling (4.2)-(6.15)-(7.13) for $\alpha \in \mathbb{N}^{*}$, we get

$$
\left\{\begin{array}{l}
\|u\|_{\mathbf{F}_{\lambda}^{\alpha}(T)} \lesssim\|u\|_{\mathbf{B}_{\lambda}^{\alpha}(T)}+\|f\|_{\mathbf{N}_{\lambda}^{\alpha}(T)} \\
\left\|\left.\partial_{x}(u v)\right|_{\mathbf{N}_{\lambda}^{\alpha}(T)} \lesssim\right\| u\left\|_{\mathbf{F}_{\lambda}^{\alpha}(T)}\right\| v\left\|_{\mathbf{F}_{\lambda}(T)}+\right\| u\left\|_{\mathbf{F}_{\lambda}(T)}\right\| v \|_{\mathbf{F}_{\lambda}^{\alpha}(T)} \\
\|u\|_{\mathbf{B}_{\lambda}^{\alpha}(T)}^{2} \stackrel{\lesssim}{\lesssim}\left\|u_{0}\right\|_{\mathbf{E}_{\lambda}^{\alpha}}^{2}+\|u\|_{\mathbf{F}_{\lambda}(T)}\|u\|_{\mathbf{F}_{\lambda}^{\alpha}(T)}^{2}
\end{array}\right.
$$

Thus, combining those estimates first with $\alpha=1$, we deduce that

$$
\mathcal{X}_{\lambda, 1}\left(T^{\prime}\right)^{2} \leqslant c_{1}\left\|u_{0}\right\|_{\mathbf{E}_{\lambda}}^{2}+c_{2}\left(\mathcal{X}_{\lambda, 1}\left(T^{\prime}\right)^{3}+\mathcal{X}_{\lambda, 1}\left(T^{\prime}\right)^{4}\right)
$$

Let us remind here that the constants appearing in (4.2)-(6.15)-(7.13) do not depend on $\lambda \geqslant 1$, so neither does (8.6). Thus, using lemma 8.3 below and a continuity argument, we get that there exists $\left.\left.T_{0}=T_{0}\left(\epsilon_{0}\right) \in\right] 0 ; 1\right]$ such that $\mathcal{X}_{\lambda, 1}(T) \leqslant 2 c_{0} \epsilon_{0}$ for $T \in\left[0 ; T_{0}\right]$. Thus, if we choose $\epsilon_{0}$ small enough such that

$$
2 c_{2} c_{0} \epsilon_{0}+4 c_{2} c_{0}^{2} \epsilon_{0}^{2}<\frac{1}{2}
$$

then

$$
\mathcal{X}_{\lambda, 1}(T) \lesssim\left\|u_{0}\right\|_{\mathbf{E}_{\lambda}}
$$

for $T \in\left[0 ; T_{0}\right]$.

(8.3) for $\alpha=1$ then follows from (4.2). 
Next, substituting the estimate obtained above in (8.5), we infer that for $\alpha=2,3$

$$
\mathcal{X}_{\lambda, \alpha}(T)^{2} \leqslant c_{\alpha}\left\|u_{0}\right\|_{\mathbf{E}_{\lambda}^{\alpha}}^{2}+\widetilde{c_{\alpha}} \epsilon_{0} \mathcal{X}_{\lambda, \alpha}(T)^{2}
$$

which in turn, up to chosing $\epsilon_{0}$ even smaller such that $\widetilde{c_{\alpha}} \epsilon_{0}<1 / 2$, gives $(8.3)$ for $\alpha=2,3$.

To reach $T=1$, we just have to use (8.3) with $\alpha=3$ along with (3.16), and then extend the lifespan of $u$ by using proposition 8.1 a finite number of times.

Therefore it remains to prove the following lemma :

\section{Lemma 8.3}

Let $T \in] 0 ; 1]$ and $u \in \mathcal{C}\left([-T ; T], \mathbf{E}_{\lambda}^{\infty}\right)$. Then $\mathcal{X}_{\lambda, 1}:[0 ; T] \rightarrow \mathbb{R}$, defined in (8.4), is continuous and nondecreasing, and furthermore

$$
\lim _{T^{\prime} \rightarrow 0} \mathcal{X}_{\lambda, 1}\left(T^{\prime}\right) \leqslant c_{0}\left\|u_{0}\right\|_{\mathbf{E}_{\lambda}}
$$

where $c_{0}>0$ is a constant independent of $\lambda$.

\section{Proof :}

From the definition of $\mathbf{B}_{\lambda}(T)(3.4)$ it is clear that for $u \in \mathcal{C}\left([-T ; T], \mathbf{E}_{\lambda}^{\infty}\right)$, $T^{\prime} \mapsto\|u\|_{\mathbf{B}_{\lambda}\left(T^{\prime}\right)}$ is nondecreasing and continuous and satisfies

$$
\lim _{T^{\prime} \rightarrow 0}\|u\|_{\mathbf{B}_{\lambda}\left(T^{\prime}\right)} \lesssim\left\|u_{0}\right\|_{\mathbf{E}_{\lambda}}
$$

where the constant only depends on the choice of the dyadic partition of unity.

Thus it remains to prove that for all $v \in \mathcal{C}\left([-T ; T], \mathbf{E}_{\lambda}^{\infty}\right), T^{\prime} \mapsto\|v\|_{\mathbf{N}_{\lambda}\left(T^{\prime}\right)}$ is increasing and continuous on $[0 ; T]$ and satisfies

$$
\lim _{T^{\prime} \rightarrow 0}\|v\|_{\mathbf{N}_{\lambda}\left(T^{\prime}\right)}=0
$$

The proof is the same as in [14, Lemma 6.3] or [4, Lemma 8.1] : first, for $M>0$ and $T^{\prime} \in[0 ; T]$, take an extension $v_{M}$ of $P_{M} v$ outside of $[-T ; T]$, then using the definition of $N_{\lambda, M}^{b_{1}}$ we get

$$
\left\|P_{M} v\right\|_{N_{\lambda, M}^{b_{1}}\left(T^{\prime}\right)} \lesssim\left\|\chi_{T^{\prime}}(t) v_{M}\right\|_{N_{\lambda, M}^{b_{1}}} \lesssim\left\|p \cdot \mathcal{F}\left\{\chi_{T^{\prime}}(t) v_{M}\right\}\right\|_{L^{2}}
$$

Using the Littlewood-Paley theorem, we obtain the bound

$$
\begin{aligned}
& \|v\|_{\mathbf{N}_{\lambda}\left(T^{\prime}\right)}=\left(\sum_{M>0}(1 \vee M)^{2}\left\|P_{M} v\right\|_{N_{\lambda, M}^{b_{1}}\left(T^{\prime}\right)}^{2}\right)^{1 / 2} \\
& \lesssim\left(\sum_{M>0}(1 \vee M)^{2}\left\|p \cdot \mathcal{F}\left\{\chi_{T^{\prime}}(t) v_{M}\right\}\right\|_{L^{2}}^{2}\right)^{1 / 2} \\
& \lesssim\left\|\chi_{T^{\prime}} v\right\|_{L_{T}^{2} \mathbf{E}_{\lambda}} \lesssim\left(T^{\prime}\right)^{1 / 2}\|v\|_{L_{T}^{\infty} \mathbf{E}_{\lambda}}
\end{aligned}
$$

This proves (8.7) and the continuity at $T^{\prime}=0$. The nondecreasing property follows from the definition of $\|\cdot\|_{Y\left(T^{\prime}\right)}(3.1)$. It remains to prove the continuity in $\left.\left.T_{0} \in\right] 0 ; T\right]$. 
Let $\epsilon>0$. If we define for $u_{0} \in L^{2}(\mathbb{R} \times \mathbb{T})$ and $L>0$,

$$
\mathcal{P}_{L} u_{0}:=\mathcal{F}^{-1}\left\{\chi_{L}(\omega(\xi, q)) \widehat{u_{0}}\right\}
$$

then by monotone convergence theorem we can take $L$ large enough such that

$$
\left\|\left(\mathrm{Id}-\mathcal{P}_{L}\right) v\right\|_{\mathbf{N}_{\lambda}\left(T_{0}\right)}<\epsilon
$$

Then it suffices to show that there exists $\delta_{0}>0$ such that for $r \in\left[1-\delta_{0} ; 1+\delta_{0}\right]$,

$$
\left|\left\|\left.v_{L}\right|_{\mathbf{N}_{\lambda}\left(T_{0}\right)}-\right\| v_{L} \|_{\mathbf{N}_{\lambda}\left(r T_{0}\right)}\right|<\epsilon
$$

Thus we may assume $v=\mathcal{P}_{L} v$ in the sequel. In particular, $P_{M} v=0$ if $M^{3} \gtrsim L$.

As in [9], we define for $r$ close to 1 the scaling operator

$$
D_{r}(v)(t, x, y):=v(t / r, x, y)
$$

Proceeding as in (8.8), we have

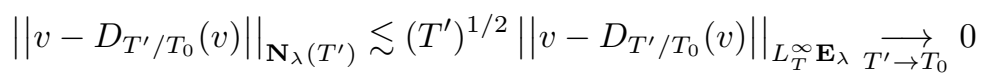

where we use that $v \in \mathcal{C}\left([-T ; T], \mathbf{E}_{\lambda}\right)$ to get the convergence.

Consequently, we are left with proving

$$
\|v\|_{\mathbf{N}_{\lambda}\left(T_{0}\right)} \leqslant \liminf _{r \rightarrow 1}\left\|D_{r}(v)\right\|_{\mathbf{N}_{\lambda}\left(r T_{0}\right)}
$$

and

$$
\limsup _{r \rightarrow 1}\left\|D_{r}(v)\right\|_{\mathbf{N}_{\lambda}\left(r T_{0}\right)} \leqslant\|v\|_{\mathbf{N}_{\lambda}\left(T_{0}\right)}
$$

Let us begin with (8.9). Fixing $\widetilde{\epsilon}>0$ and $r \in[1 / 2 ; 2]$, for any $M \in 2^{\mathbb{Z}}, M^{3} \lesssim L$, we can choose an extension $v_{M, r}$ of $P_{M} D_{r}(v)$ satisfying $v_{M, r} \equiv P_{M} D_{r}(v)$ on $\left[-r T_{0} ; r T_{0}\right]$ and

$$
\left\|v_{M, r}\right\|_{N_{\lambda, M}^{b_{1}}} \leqslant\left\|P_{M} D_{r}(v)\right\|_{N_{\lambda, M}^{b_{1}}\left(r T_{0}\right)}+\widetilde{\epsilon}
$$

Since $D_{1 / r}\left(v_{M, r}\right) \equiv P_{M} v$ on $\left[-T_{0} ; T_{0}\right]$, it defines an extension of $P_{M} v$ and thus

$$
\|v\|_{\mathbf{N}_{\lambda}\left(T_{0}\right)} \leqslant\left(\sum_{M \lesssim L^{1 / 3}}(1 \vee M)^{2}\left\|D_{1 / r}\left(v_{M, r}\right)\right\|_{N_{\lambda, M}^{b_{1}}}^{2}\right)^{1 / 2}
$$

Finally, it remains to prove that

$$
\left\|D_{1 / r}\left(v_{M, r}\right)\right\|_{N_{\lambda, M}^{b_{1}}} \leqslant \psi(r)\left\|v_{M, r}\right\|_{N_{\lambda, M}^{b_{1}}}
$$

to get (8.9), where $\psi$ is a continuous function defined on a neighborhood of $r=1$ and satisfying $\lim _{r \rightarrow 1} \psi(r)=1$.

From the definition of $N_{\lambda, M}^{b_{1}}$, we have

$$
\begin{aligned}
& \left\|D_{1 / r}\left(v_{M, r}\right)\right\|_{N_{\lambda, M}^{b_{1}}} \\
& \quad=\sup _{t_{M} \in \mathbb{R}}\left\|(\tau-\omega+i(1 \vee M))^{-1} p \mathcal{F}\left\{\chi_{(1 \vee M)^{-1}}\left(\cdot-t_{M}\right) D_{1 / r}\left(v_{M, r}\right)\right\}\right\|_{X_{\lambda, M}^{b_{1}}}
\end{aligned}
$$


and a computaton gives

$$
\chi_{(1 \vee M)^{-1}}\left(\cdot-t_{M}\right) D_{1 / r}\left(v_{M, r}\right)=D_{1 / r}\left(\chi_{r(1 \vee M)^{-1}}\left(\cdot-r t_{M}\right) v_{M, r}\right)
$$

so that

$$
\mathcal{F}\left\{\chi_{(1 \vee M)^{-1}}\left(\cdot-t_{M}\right) D_{1 / r}\left(v_{M, r}\right)\right\}=r^{-1} D_{r}\left(\mathcal{F}\left\{\chi_{r(1 \vee M)^{-1}}\left(\cdot-r t_{M}\right) v_{M, r}\right\}\right)
$$

Thus, using the definition of $X_{\lambda, M}^{b_{1}}$, the left-hand side of (8.11) equals

$$
\begin{aligned}
r^{-1 / 2} \sup _{\widetilde{t_{M}} \in \mathbb{R}} \sum_{K \geqslant 1} K^{1 / 2} \beta_{M, K}^{b_{1}} & \\
& \left\|(r \tau-\omega+i(1 \vee M))^{-1} \rho_{K}(r \tau-\omega) p \mathcal{F}\left\{\chi_{r(1 \vee M)^{-1}}\left(\cdot-\widetilde{t_{M}}\right) v_{M, r}\right\}\right\|_{L^{2}}
\end{aligned}
$$

Now, for $r \sim 1$, we observe that for $K \geqslant 10^{10} L$, we have $|\tau| \sim|\tau-\omega| \sim|r \tau-\omega| \sim K$, whereas for $K \lesssim L$ we have $|\tau|,|\tau-\omega|$ and $|r \tau-\omega| \lesssim L$.

Thus,

$$
\begin{aligned}
\mid \frac{1}{(r \tau-\omega)^{2}+(1 \vee M)^{2}}-\frac{1}{(\tau-\omega)^{2}+(1} & \vee M)^{2} \mid \\
& \lesssim|1-r|(1 \vee L)^{2} \cdot \frac{1}{(\tau-\omega)^{2}+(1 \vee M)^{2}}
\end{aligned}
$$

and the use of the mean value theorem provides

$$
\left|\rho_{K}(r \tau-\omega)-\rho_{K}(\tau-\omega)\right| \lesssim|1-r|\left\{\begin{array}{l}
\sum_{K^{\prime} \sim K} \rho_{K^{\prime}}(\tau-\omega) \text { if } K \geqslant 10^{10} L \\
K^{-1} L \sum_{K^{\prime} \lesssim L} \rho_{K^{\prime}}(\tau-\omega) \text { if } K \lesssim L
\end{array}\right.
$$

Combining all the estimates above, we get the bound

$$
\begin{aligned}
& \left\|D_{1 / r}\left(v_{M, r}\right)\right\|_{N_{\lambda, M}^{b_{1}}} \\
& \quad \leqslant \widetilde{\psi}(r) \sup _{\bar{t}_{M}}\left\|(\tau-\omega+i(1 \vee M))^{-1} p \mathcal{F}\left\{\chi_{r(1 \vee M)^{-1}}\left(\cdot-\widetilde{t_{M}}\right) v_{M, r}\right\}\right\|_{X_{\lambda, M}^{b_{1}}}
\end{aligned}
$$

where $\widetilde{\psi}(r)=r^{-1 / 2}\left(1+C(1 \vee L)^{2}|r-1|\right)^{3 / 2} \underset{r \rightarrow 1}{\longrightarrow} 1$.

It remains to treat the time localization term : using the fundamental theorem of calculus, we have

$$
F\left(t-\widetilde{t_{M}}\right):=\chi_{r(1 \vee M)^{-1}}\left(t-\widetilde{t_{M}}\right)-\chi_{(1 \vee M)^{-1}}\left(t-\widetilde{t_{M}}\right)=\int_{1}^{r^{-1}} s^{-1} \varphi\left(s(1 \vee M)\left(t-\widetilde{t_{M}}\right)\right) \mathrm{d} s
$$

with $\varphi(t):=t \chi^{\prime}(t)$. In particular, for $r \in[1 / 2 ; 2]$, from the support property of $\chi$, the support of $F\left(\cdot-\widetilde{t_{M}}\right)$ is included in $\left[\widetilde{t_{M}}-4(1 \vee M) ; \widetilde{t_{M}}+4(1 \vee M)\right]$, thus we can represent

$$
F\left(t-\widetilde{t_{M}}\right)=F\left(t-\widetilde{t_{M}}\right) \sum_{|\ell| \leqslant 4} \gamma\left((1 \vee M)\left(t-\widetilde{t_{M}}-\ell\right) \chi_{(1 \vee M)^{-1}}\left(t-\widetilde{t_{M}}-\ell(1 \vee M)^{-1}\right)\right.
$$


where $\gamma$ is a smooth partition of unity with supp $\gamma \subset[-1 ; 1]$ satisfying $\forall x \in \mathbb{R}, \sum_{\ell \in \mathbb{Z}} \gamma(x-\ell)=1$.

Now, using Minkowski's integral inequality to deal with the integral in $s$, the right-handside of (8.14) is less than

$$
\begin{gathered}
\widetilde{\psi}(r)\left(\left\|v_{M, r}\right\|_{N_{\lambda, M}^{b_{1}}}+\int_{I(r)} s^{-1} \underset{\sup _{M}}{|\ell| \leqslant 4} \|(\tau-\omega+i(1 \vee M))^{-1} p \mathcal{F}\left\{\varphi \left(s(1 \vee M)\left(t-\widetilde{t_{M}}\right)\right.\right.\right. \\
\left.\left.\cdot \gamma_{(1 \vee M)^{-1}}\left(t-\widetilde{t_{M}}-(1 \vee M)^{-1} \ell\right) \chi_{(1 \vee M)^{-1}}\left(t-\widetilde{t_{M}}-(1 \vee M)^{-1} \ell\right) v_{M, r}\right\} \|_{X_{\lambda, M}^{b_{1}}} \mathrm{~d} s\right)
\end{gathered}
$$

with $I(r)=\left[1 ; r^{-1}\right]$ if $r \in[1 / 2 ; 1]$ and $I(r)=\left[r^{-1} ; 1\right]$ if $r \in[1 ; 2]$.

Since $\varphi(t)=t \chi^{\prime}(t)$ and $\gamma$ are smooth, twice the use of (3.10) and (3.11) (with $K_{0}=$ $s(1 \vee M)$ and $K_{0}=(1 \vee M)$ respectively) provides the final bound

$$
\left\|\left.D_{1 / r}\left(v_{M, r}\right)\right|_{N_{\lambda, M}^{b_{1}}} \leqslant \widetilde{\psi}(r)(1+C|\ln (r)|)\right\| v_{M, r} \|_{N_{\lambda, M}^{b_{1}}}
$$

(here we used that the implicit constant in (3.10) and (3.11) are independent of $s$ ). This concludes the proof of (8.9).

To prove (8.10), as before we may assume $v=\mathcal{P}_{L} v$. Given $\widetilde{\epsilon}>0$ for any $M>0$ we take an extension $v_{M}$ of $P_{M} v$ outside of $\left[-T_{0} ; T_{0}\right]$ and satisfying $\left\|v_{M}\right\|_{N_{\lambda, M}^{b_{1}}} \leqslant\left\|P_{M} v\right\|_{N_{\lambda, M}^{b_{1}}\left(T_{0}\right)}+\tilde{\epsilon}$. Then for $r \in[-1 / 2 ; 2], D_{r}\left(v_{M}\right)$ defines an extension of $P_{M} D_{r}(v)$ outside of $\left[-r T_{0} ; r T_{0}\right]$. Then, since in the proof of (8.15) we did not used the dependence in $r$ of $v_{M, r}$, the same estimate actually holds for $v_{M}$, and thus

$$
\left\|\left.D_{r}\left(v_{M}\right)\right|_{N_{\lambda, M}^{b_{1}}} \leqslant \psi(1 / r)\right\| v_{M} \|_{N_{\lambda, M}^{b_{1}}}
$$

which is enough for (8.10) and thus concludes the proof of the lemma.

\subsection{Global well-posedness for smooth data}

In view of the previous proposition, theorem 1.1 (a) follows from the conservation of the energy.

Indeed, take $u_{0} \in \mathbf{E}_{\lambda}^{\infty}$ satisfying

$$
\left\|u_{0}\right\|_{\mathbf{E}_{\lambda}} \leqslant \epsilon_{1} \leqslant \epsilon_{0}
$$

and let $T^{*}:=\sup \left\{T \geqslant 1,\|u(T)\|_{\mathbf{E}_{\lambda}}<+\infty\right\}$ where $u$ is the unique maximal solution of (1.6) given by proposition 8.2. Then, using the anisotropic Sobolev estimate (see [24, Lemma 2.5])

$$
\int_{\mathbb{R} \times \mathbb{T}_{\lambda}} u_{0}(x, y)^{3} \mathrm{~d} x \mathrm{~d} y \leqslant 2\left\|u_{0}\right\|_{L^{2}}^{3 / 2}\left\|\partial_{x} u_{0}\right\|_{L^{2}}\left\|\partial_{x}^{-1} \partial_{y} u_{0}\right\|_{L^{2}}^{1 / 2}
$$

we have for $T<T^{*}$

$$
\begin{aligned}
& \|u(T)\|_{\mathbf{E}_{\lambda}}^{2}=\mathcal{M}(u(T))+\mathcal{E}(u(T))+\frac{1}{3} \int_{\mathbb{R} \times \mathbb{T}} u^{3}(T, x, y) \mathrm{d} x \mathrm{~d} y \\
& \leqslant \mathcal{M}(u(T))+\mathcal{E}(u(T))+2\|u(T)\|_{L^{2}}^{3 / 2}\left\|\partial_{x} u(T)\right\|_{L^{2}}\left\|\partial_{x}^{-1} \partial_{y} u(T)\right\|_{L^{2}}^{1 / 2} \\
& \leqslant \mathcal{M}(u(T))+\mathcal{E}(u(T))+2 \mathcal{M}(u(T))\|u(T)\|_{\mathbf{E}_{\lambda}}^{2}
\end{aligned}
$$


Thus, from the conservation of $\mathcal{M}$ and $\mathcal{E}$ (as $u$ is a smooth solution), we finally obtain

$$
\|u(T)\|_{\mathbf{E}_{\lambda}}^{2} \lesssim \mathcal{M}\left(u_{0}\right)+\mathcal{E}\left(u_{0}\right)<+\infty
$$

for any $T<T^{*}$ provided $\epsilon_{1}^{2}<1 / 4$, from which we get $T^{*}=+\infty$.

Finally, let us notice that equation (1.6) admits the scaling

$$
u_{\lambda}(t, x, y):=\lambda^{-1} u\left(\lambda^{-3 / 2} t, \lambda^{-1 / 2} x, \lambda^{-1} y\right),(x, y) \in \mathbb{R} \times \mathbb{T}_{\lambda \lambda_{0}}
$$

meaning that $u_{\lambda}$ is a solution of (1.6) on $\left[-\lambda^{3 / 2} T ; \lambda^{3 / 2} T\right] \times \mathbb{R} \times \mathbb{T}_{\lambda \lambda_{0}}$ if and only if $u$ is a solution of $(1.6)$ on $[-T ; T] \times \mathbb{R} \times \mathbb{T}_{\lambda_{0}}$. Moreover,

$$
\left.\left\|u_{\lambda}(0)||_{\mathbf{E}_{\lambda \lambda_{0}}} \lesssim \lambda^{-1 / 4}\right\| u(0)\right|_{\mathbf{E}_{\lambda_{0}}}
$$

Thus, take $u_{0} \in \mathbf{E}_{\lambda_{0}}^{\infty}$. If $\left\|u_{0}\right\|_{\mathbf{E}_{\lambda_{0}}}>\epsilon_{1}$, then there exists

$$
\lambda=\lambda\left(\left\|u_{0}\right\|_{\mathbf{E}_{\lambda_{0}}}\right) \sim \epsilon_{1}^{-4}\left\|u_{0}\right\|_{\mathbf{E}_{\lambda_{0}}}^{-4}>1
$$

such that $\left\|u_{0, \lambda}\right\|_{\mathbf{E}_{\lambda \lambda_{0}}^{\alpha}} \leqslant \epsilon_{1}$ (since $\epsilon_{1}>0$ is independent of $\left.\lambda \geqslant 1\right)$. Thus, if $u_{\lambda} \in \mathcal{C}\left(\mathbb{R}, \mathbf{E}_{\lambda \lambda_{0}}^{\infty}\right)$ is the unique global solution associated with $u_{0, \lambda}$ satisfying (8.16), then

$$
u(t, x, y):=\lambda u_{\lambda}\left(\lambda^{3 / 2} t, \lambda^{1 / 2} x, \lambda y\right) \in \mathcal{C}\left(\mathbb{R}, \mathbf{E}_{\lambda_{0}}^{\infty}\right)
$$

is the unique global solution associated with $u_{0}$.

The rest of the section is devoted to the proof of theorem 1.1 (b).

\subsection{Lipschitz bound for the difference of small data solutions}

Let $T>0, u_{0}, v_{0} \in \mathbf{E}_{\lambda}$ and $u, v$ in the class (1.8) be the corresponding solutions of the Cauchy problems (1.6). As before, up to rescaling and using the conservation of $\mathcal{M}$ and $\mathcal{E}$, it suffices to prove uniqueness for $T=1$ and

$$
\left\|u_{0}\right\|_{\mathbf{E}_{\lambda}},\left\|v_{0}\right\|_{\mathbf{E}_{\lambda}} \leqslant \epsilon_{2} \leqslant \epsilon_{0}
$$

Set $w:=u-v$. Then $w$ is also in the class (8.2) and solves the equation

$$
\partial_{t} w+\partial_{x}^{3} w-\partial_{x}^{-1} \partial_{y} w+\partial_{x}\left(w \cdot \frac{u+v}{2}\right)=0
$$

on $[-1 ; 1] \times \mathbb{R} \times \mathbb{T}_{\lambda}$. Then, since $u_{0}, v_{0}$ satisfy (8.2), using (8.3) and then (3.17), (4.10)(6.17)-(7.23), we obtain for $\epsilon_{2}$ small enough

$$
\left\|\left.w\right|_{L_{[-1 ; 1]}^{\infty} L_{x y}^{2}} \lesssim\right\| w\left\|_{\overline{\mathbf{F}_{\lambda}}(1)} \lesssim\right\| u_{0}-v_{0} \|_{L^{2}}
$$

from which we get $u \equiv v$ on $[-1 ; 1]$ if $u_{0}=v_{0}$. 


\subsection{Global well-posedness in the energy space}

In this subsection we end the proof of theorem 1.1 (b). We proceed as in [9, Section 4].

Take $T>0$, and let $u_{0} \in \mathbf{E}_{\lambda}$ and $\left(u_{0, n}\right) \in\left(\mathbf{E}_{\lambda}^{\infty}\right)^{\mathbb{N}}$ such that $\left(u_{0, n}\right)$ converges to $u_{0}$ in $\mathbf{E}_{\lambda}$. Again, up to rescaling we can assume $\left\|u_{0}\right\|_{\mathbf{E}_{\lambda}} \leqslant \epsilon \leqslant \epsilon_{2}$ and $\left\|u_{0, n}\right\|_{\mathbf{E}_{\lambda}} \leqslant \epsilon \leqslant \epsilon_{2}$. Using again the conservation of $\mathcal{M}$ and $\mathcal{E}$, it then suffices to prove that $\left(\Phi^{\infty}\left(u_{0, n}\right)\right) \in\left(\mathcal{C}\left([-1 ; 1], \mathbf{E}_{\lambda}^{\infty}\right)\right)^{\mathbb{N}}$ is a Cauchy sequence in $\mathcal{C}\left([-1 ; 1], \mathbf{E}_{\lambda}\right)$.

For a fixed $M>1$ and $m, n \in \mathbb{N}$, we can split

$$
\begin{aligned}
& \left\|\Phi^{\infty}\left(u_{0, m}\right)-\Phi^{\infty}\left(u_{0, n}\right)\right\|_{L_{1}^{\infty} \mathbf{E}_{\lambda}} \leqslant\left\|\Phi^{\infty}\left(u_{0, m}\right)-\Phi^{\infty}\left(P_{\leqslant M} u_{0, m}\right)\right\|_{L_{1}^{\infty} \mathbf{E}_{\lambda}} \\
& \quad+\left\|\Phi^{\infty}\left(P_{\leqslant M} u_{0, m}\right)-\Phi^{\infty}\left(P_{\leqslant M} u_{0, n}\right)\right\|_{L_{1}^{\infty} \mathbf{E}_{\lambda}}+\left\|\Phi^{\infty}\left(P_{\leqslant M} u_{0, n}\right)-\Phi^{\infty}\left(u_{0, n}\right)\right\|_{L_{1}^{\infty} \mathbf{E}_{\lambda}}
\end{aligned}
$$

Since

$$
\left\|S_{T}^{\infty}\left(P_{\leqslant M} u_{0, n}\right)\right\|_{L_{1}^{\infty} \mathbf{E}_{\lambda}^{\alpha}} \leqslant C(\alpha, M)
$$

thanks to (1.7), the middle term is then controled with the analogous of (8.1) for the difference equation along with a Sobolev inequality with $\alpha$ large enough, which gives

$$
\left\|\Phi^{\infty}\left(P_{\leqslant M} u_{0, m}\right)-\Phi^{\infty}\left(P_{\leqslant M} u_{0, n}\right)\right\|_{L_{1}^{\infty} \mathbf{E}_{\lambda}} \leqslant C(M)\left\|u_{0, m}-u_{0, n}\right\|_{\mathbf{E}_{\lambda}}
$$

Therefore it remains to treat the first and last terms. A use of (3.16) provides

$$
\left\|\Phi^{\infty}\left(u_{0, m}\right)-\Phi^{\infty}\left(P_{\leqslant M} u_{0, m}\right)\right\|_{L_{1}^{\infty} \mathbf{E}_{\lambda}} \lesssim\left\|\Phi^{\infty}\left(u_{0, m}\right)-\Phi^{\infty}\left(P_{\leqslant M} u_{0, m}\right)\right\|_{\mathbf{F}_{\lambda}(1)}
$$

and thus we have to estimate difference of solutions in $\mathbf{F}_{\lambda}(1)$. Let us write $u_{1}:=\Phi^{\infty}\left(u_{0, m}\right)$, $u_{2}:=\Phi^{\infty}\left(P_{\leqslant M} u_{0, m}\right)$ and $v:=u_{1}-u_{2}$.

Using (4.2) and (6.15) combined with (8.3) we obtain the bound

$$
\|v\|_{\mathbf{F}_{\lambda}(1)} \lesssim\|v\|_{\mathbf{B}_{\lambda}(1)}+\|v\|_{\mathbf{F}_{\lambda}(1)} \epsilon
$$

Therefore, taking $\epsilon$ small enough, it suffices to control $\|v\|_{\mathbf{B}_{\lambda}(1)}$. Using the definition of $\mathbf{B}_{\lambda}(1)(3.4)$, we see that

$$
\|v\|_{\mathbf{B}_{\lambda}(1)} \leqslant\left\|P_{\leqslant 1} v_{0}\right\|_{\mathbf{E}_{\lambda}}+\left\|P_{\geqslant 2} v\right\|_{\mathbf{B}_{\lambda}(1)}
$$

Now, in view of the definition of $\mathbf{B}_{\lambda}(1)$ and $\overline{\mathbf{B}_{\lambda}}(1)$, we have

$$
\left\|P_{\geqslant 2} v\right\|_{\mathbf{B}_{\lambda}(1)} \sim\left\|\partial_{x} P_{\geqslant 2} v\right\|_{\overline{\mathbf{B}_{\lambda}}(1)}+\|\left.\partial_{x}^{-1} \partial_{y} P_{\geqslant 2} v\right|_{\overline{\mathbf{B}_{\lambda}}(1)}
$$

Combining this remark with the previous estimates, we finally get the bound

$$
\|v\|_{\mathbf{F}_{\lambda}(1)} \lesssim\left\|v_{0}\right\|_{\mathbf{E}_{\lambda}}+\left\|P_{\geqslant 2} \partial_{x} v\right\|_{\overline{\mathbf{B}_{\lambda}}(1)}+\|\left. P_{\geqslant 2} \partial_{x}^{-1} \partial_{y} v\right|_{\overline{\mathbf{B}_{\lambda}}(1)}
$$

We now define $U:=P_{H i g h} \partial_{x} v$ and $V:=P_{H i g h} \partial_{x}^{-1} \partial_{y} v$. We begin by writing down the equations satisfied by $U$ and $V$ :

$$
\begin{aligned}
& \partial_{t} U+\partial_{x}^{3} U-\partial_{x}^{-1} \partial_{y}^{2} U=P_{\text {High }}\left(-u_{1} \cdot \partial_{x} U\right)+P_{H i g h}\left(-P_{\text {Low }} u_{1} \cdot \partial_{x}^{2} P_{\text {Low }} v\right) \\
& \quad+P_{\text {High }}\left(-P_{\text {High }} u_{1} \cdot \partial_{x}^{2} P_{\text {Low }} v\right)+P_{\text {High }}\left(-\partial_{x} v \cdot \partial_{x}\left(u_{1}+u_{2}\right)\right)+P_{\text {High }}\left(-v \cdot \partial_{x}^{2} u_{2}\right)
\end{aligned}
$$


and

$$
\begin{aligned}
\partial_{t} V+\partial_{x}^{3} V-\partial_{x}^{-1} \partial_{y}^{2} V= & P_{\text {High }}\left(-u_{1} \cdot \partial_{x} V\right)+P_{\text {High }}\left(-P_{\text {Low }} u_{1} \cdot \partial_{x} P_{\text {Low }} \partial_{x}^{-1} \partial_{y} v\right) \\
& +P_{\text {High }}\left(-P_{\text {High }} u_{1} \cdot \partial_{x} P_{\text {Low }} \partial_{x}^{-1} \partial_{y} v\right)+P_{\text {High }}\left(-v \cdot \partial_{y} u_{2}\right)
\end{aligned}
$$

Let us look at (8.22). We set $h:=-P_{\text {Low }} u_{1} \cdot \partial_{x}^{2} P_{\text {Low }} v, w_{1}:=-P_{\text {High }} u_{1}, w_{1}^{\prime}:=\partial_{x}^{2} P_{\text {Low }} v$, $w_{2}:=-\partial_{x} v, w_{2}^{\prime}:=\partial_{x}\left(u_{1}+u_{2}\right)$ and $w_{3}:=-v, w_{3}^{\prime}:=\partial_{x}^{2} u_{2}$. Since $u_{1}, u_{2} \in \mathbf{F}_{\lambda}(1)$ we have $v \in \mathbf{F}_{\lambda}(1)$, thus $h, w_{i}$ and $w_{i}^{\prime}$ satisfy the assumptions of (7.25). Thence we infer

$$
\begin{aligned}
& \|U\|_{\overline{\mathbf{B}_{\lambda}}(1)}^{2} \lesssim\left\|\partial_{x} v_{0}\right\|_{L_{\lambda}^{2}}^{2}+\left\|u_{1}\right\|_{\mathbf{F}_{\lambda}(1)}\|U\|_{\overline{\mathbf{F}_{\lambda}}(1)}^{2} \\
& \quad+\|U\|_{\overline{\mathbf{F}_{\lambda}}(1)}\left(\left\|P_{H i g h} u_{1}||_{\overline{\mathbf{F}_{\lambda}}(1)}\right\| \partial_{x}^{2} P_{L o w} v \|_{\overline{\mathbf{F}_{\lambda}}(1)}\right. \\
& \left.\quad+\left\|\partial_{x} v\right\|_{\overline{\mathbf{F}_{\lambda}}(1)}\left\|\partial_{x}\left(u_{1}+u_{2}\right)\right\|_{\overline{\mathbf{F}_{\lambda}}(1)}+\left\|v||_{\overline{\mathbf{F}_{\lambda}(1)}}\right\| \partial_{x}^{2} u_{2} \|_{\overline{\mathbf{F}_{\lambda}(1)}}\right)
\end{aligned}
$$

Therefore, using (8.3) and (8.20), the previous estimate reads

$$
\begin{aligned}
\|U\|_{\overline{\mathbf{B}_{\lambda}}(1)}^{2} \lesssim\left\|v_{0}\right\|_{\mathbf{E}_{\lambda}}^{2}+\epsilon\|U\|_{\overline{\mathbf{F}_{\lambda}}(1)}^{2}+\|U\|_{\overline{\mathbf{F}_{\lambda}(1)}}\left(\epsilon\left\|v_{0}\right\|_{L_{\lambda}^{2}}\right. & \\
& \left.+\|v\|_{\mathbf{F}_{\lambda}(1)} \epsilon+\left\|v_{0}\right\|_{L_{\lambda}^{2}}\left\|u_{2}\right\|_{\mathbf{F}_{\lambda}^{2}(1)}\right)
\end{aligned}
$$

Proceeding similarly for $V$, we obtain the estimate

$$
\begin{aligned}
\|V\|_{\overline{\mathbf{B}_{\lambda}}(1)}^{2} \lesssim & \left\|\partial_{x}^{-1} \partial_{y} v_{0}\right\|_{L_{\lambda}^{2}}^{2}+\left\|u_{1}\right\|_{\mathbf{F}_{\lambda}(1)}\|V\|_{\overline{\mathbf{F}_{\lambda}}(1)}^{2}+\|V\| \|_{\overline{\mathbf{F}_{\lambda}}(1)} \\
& \cdot\left(\left\|P_{H i g h} u_{1}\right\|_{\overline{\mathbf{F}_{\lambda}}(1)}\left\|\left.\partial_{x} P_{\text {Low }} \partial_{x}^{-1} \partial_{y} v\right|_{\overline{\mathbf{F}_{\lambda}(1)}}+\right\| v\left\|_{\overline{\mathbf{F}_{\lambda}}(1)}\right\| \partial_{x} \partial_{x}^{-1} \partial_{y} u_{2} \|_{\overline{\mathbf{F}_{\lambda}}(1)}\right)
\end{aligned}
$$

after applying (7.25). Again, a use of (8.3) and (8.20) gives

$$
\|V\|_{\mathbf{B}_{\lambda}(1)}^{2} \lesssim\left\|v_{0}\right\|_{\mathbf{E}_{\lambda}}^{2}+\left.\epsilon\|V\|\right|_{\mathbf{F}_{\lambda}(1)} ^{2}+\|V\|_{\overline{\mathbf{F}_{\lambda}}(1)}\left(\epsilon\|v\|_{\mathbf{F}_{\lambda}(1)}+\left\|v_{0}\right\|_{L_{\lambda}^{2}}\left\|u_{2}\right\|_{\mathbf{F}_{\lambda}^{2}(1)}\right)
$$

Combining the estimates for $U$ and $V$ along with (8.21), we get the final bound

$$
\|v\|_{\mathbf{F}_{\lambda}(1)} \lesssim\left\|v_{0}\right\|_{\mathbf{E}_{\lambda}}+\epsilon\left\|\left.v\right|_{\mathbf{F}_{\lambda}(1)}+\right\| P_{\leqslant M} u_{0, m}\left\|_{\mathbf{E}_{\lambda}^{2}}\right\| v_{0} \|_{L_{\lambda}^{2}}
$$

since $\left\|\left.u_{2}\right|_{\mathbf{F}_{\lambda}^{2}(1)} \lesssim\right\| u_{2}(0) \|_{\mathbf{E}_{\lambda}^{2}}$ by $(8.3)$.

Taking $\epsilon$ small enough and $M>1$ large enough concludes the proof.

\section{Orbital stability of the line soliton}

In this last section, we turn to the proof of corollary 1.2. We briefly recall the main steps of [21, Section 2].

Let us remember that equation (1.6) has a Hamiltonian structure, with Hamiltonian $E(u)$. To study the orbital stability of $Q_{c}(x-c t)$, we first make a change of variable to see $Q_{c}(x)$ as a stationary solution of (1.6) rewriten in a moving frame :

$$
\partial_{t} u-c \partial_{x} u+\partial_{x}^{3} u-\partial_{x}^{-1} \partial_{y}^{2} u+u \partial_{x} u=0
$$


Equation (9.1) still has a Hamitonian structure, with the new Hamiltonian

$$
\mathcal{E}_{c}(u):=\mathcal{E}(u)+c \mathcal{M}(u)
$$

The key idea of the proof is then to show, as for the orbital stability of $Q_{c}$ under the flow of $\mathrm{KdV}[2]$, that the Hessian of $\mathcal{E}_{c}$ about $Q_{c}$ is strictly positive on the codimension-2 subspace $H:=\left\{\left\langle v, Q_{c}\right\rangle_{L^{2}}=\left\langle v, Q_{c}^{\prime}\right\rangle_{L^{2}}=0\right\}$ to get a lower bound on $\mathcal{E}_{c}\left(\Phi^{1}\left(u_{0}\right)(t)\right)-\mathcal{E}_{c}\left(Q_{c}\right)$ in term of $\left\|\Phi^{1}\left(u_{0}\right)(t)-Q_{c}\right\|_{\mathbf{E}}$

To study $D^{2} \mathcal{E}_{c}\left(Q_{c}\right)$ on $H$, we begin by computing

$$
\begin{array}{r}
\mathcal{E}_{c}\left(Q_{c}+v(t)\right)=\mathcal{E}_{c}\left(Q_{c}\right)+\left(\left\|\partial_{x} v\right\|_{L^{2}}^{2}+\left\|\partial_{x}^{-1} \partial_{y} v\right\|_{L^{2}}^{2}+c\|v\|_{L^{2}}^{2}-\int_{\mathbb{R} \times \mathbb{T}} Q_{c} \cdot v^{2} \mathrm{~d} x \mathrm{~d} y\right) \\
-\int_{\mathbb{R} \times \mathbb{T}} v^{3} \mathrm{~d} x \mathrm{~d} y
\end{array}
$$

The linear term in $v$ vanishes since $Q_{c}$ is a stationary solution.

Using the Plancherel identity in the $y$ variable, we can write the Hessian of $\mathcal{E}_{c}$ about $Q_{c}$ as the sum of the bilinear forms

$$
\frac{1}{2} D^{2} \mathcal{E}_{c}\left(Q_{c}\right)(v, v)=\sum_{k \in \mathbb{Z}} B_{c}^{k}\left(\mathcal{F}_{y} v(t, x, k), \mathcal{F}_{y} v(t, x, k)\right)
$$

with

$$
B_{c}^{k}(\widetilde{v}(x), \widetilde{v}(x))=\left\|\partial_{x} \widetilde{v}\right\|_{L^{2}}^{2}+k^{2}\left\|\partial_{x}^{-1} \widetilde{v}\right\|_{L^{2}}^{2}+c\|\widetilde{v}\|_{L^{2}}^{2}-\int_{\mathbb{R}} Q_{c} \cdot \widetilde{v}^{2} \mathrm{~d} x
$$

Observe that $B_{c}^{0}$ is the Hessian about $Q_{c}$ of the Hamiltonian associated with the $\mathrm{KdV}$ equation in a moving frame, and thus by the study in [2] $B_{c}^{0}$ is $H^{1}$ bounded from below as desired.

To treat the terms with $k \neq 0$, first make the change of test function

$$
f(x):=\partial_{x}^{-1} \mathcal{F}_{y} v(t, x, k) \in L^{2}(\mathbb{R})
$$

Then, using that $k^{2} \geqslant 1$, we can write

$$
B_{c}^{k}\left(\mathcal{F}_{y} v(t, x, k), \mathcal{F}_{y} v(t, x, k)\right) \geqslant\left\langle\mathcal{L}_{c} f, f\right\rangle
$$

where the linear operator $\mathcal{L}_{c}$ is defined as

$$
\mathcal{L}_{c}:=\partial_{x}^{4}-c \partial_{x}^{2}+\partial_{x} Q_{c} \partial_{x}+1
$$

Since $Q_{c}$ is exponentially decreasing, $\partial_{x} Q_{c} \partial_{x}$ is compact with respect to $\partial_{x}^{4}-c \partial_{x}^{2}+1$ and thus $\operatorname{Spec}_{e s s} \mathcal{L}_{c} \subset\left[1,+\infty\left[\right.\right.$. To get a lower bound on $\left\langle\mathcal{L}_{c} f, f\right\rangle$, it remains to study the existence of negative eigenvalues. Following the method of [1], a change of variables leads to consider the eigenvalue problem

$$
g^{(4)}-4\left(1-\frac{3}{\cosh ^{2}}\right) g^{\prime \prime}+3 \nu^{2} g=0
$$

where

$$
3 \nu^{2}=\frac{16}{c^{2}}\left(1-\lambda_{0}\right)
$$

and $\lambda_{0} \leqslant 0$ is the possible negative eigenvalue. Using again the exponential decreasing of $Q_{c}, g$ behaves at infinity as a solution of the linear equation

$$
h^{(4)}-4 h^{\prime \prime}+3 \nu^{2} h=0
$$


For each characteristic value $\mu$ of (9.3), there is an exact solution

$$
g_{\mu}(x):=\mathrm{e}^{\mu x}\left(\mu^{3}+2 \mu-3 \mu^{2} \tanh (x)\right)
$$

of (9.2). For these solutions to behave as $\mathrm{e}^{\mu x}$ at infinity, this requires

$$
\mu^{3}+2 \mu-3 \mu^{2}=0
$$

As $\mu$ is also a characteristic value, this implies $\mu=1$ and thus $\nu^{2}=1$ from which we finally infer

$$
c^{2}=\frac{16}{3}\left(1-\lambda_{0}\right)
$$

Consequently, there is no possible negative eigenvalue $\lambda_{0}$ if $c<c^{*}=4 / \sqrt{3}$.

Hence we have a lower $L^{2}$ bound for the bilinear form associated with $\mathcal{L}_{c}$, which provides the bound

$$
B_{c}^{k}(\widetilde{v}, \widetilde{v}) \gtrsim\left\|\partial_{x}^{-1} \widetilde{v}\right\|_{L^{2}}^{2}
$$

Linearly interpolating with the obvious bound (since $Q_{c} \leqslant 3 c$ )

$$
B_{c}^{k}(\widetilde{v}, \widetilde{v}) \geqslant\left\|\partial_{x} \widetilde{v}\right\|_{L^{2}}^{2}+\left\|\left.\partial_{x}^{-1} \widetilde{v}\right|_{L^{2}} ^{2}-2 c \mid\right\| \widetilde{v} \|_{L^{2}}^{2}
$$

yields to an $L^{2}$ lower bound for $B_{c}^{k}$, which in return provides the final bound

$$
B_{c}^{k}(\widetilde{v}, \widetilde{v}) \gtrsim\|\widetilde{v}\|_{H^{1}}^{2}+k^{2}\left\|\partial_{x}^{-1} \widetilde{v}\right\|_{L^{2}}^{2}
$$

uniformly in $k$.

The last trilinear term $\int v^{3}$ is treated with the anisotropic Sobolev inequality (8.17).

Combining all the bounds from below finally provides a control of $\|w\|_{\mathbf{E}}$ in term of $\mathcal{E}_{c}\left(Q_{c}+w_{0}\right)-\mathcal{E}_{c}\left(Q_{c}\right)$ for any $w \in H$. The end of the proof is then standard (cf. [2],[21, Section 2]).

\section{Acknowledgements.}

The author would like to thank his thesis supervisor Nikolay Tzvetkov for pointing out this problem as well as for many helpfull discussions.

\section{References}

[1] J.C. Alexander, R.L. Pego, and R.L. Sachs. On the transverse instability of solitary waves in the Kadomtsev-Petviashvili equation. Physics Letters A, 226(3):187 - 192, 1997.

[2] T. B. Benjamin. The stability of solitary waves. Proceedings of the Royal Society of London A: Mathematical, Physical and Engineering Sciences, 328(1573):153-183, 1972.

[3] Jean Bourgain. On the cauchy problem for the Kadomstev-Petviashvili equation. Geometric and Functional Analysis, 3(4):315-341, 1993.

[4] Zihua Guo and Tadahiro Oh. Non-existence of solutions for the periodic cubic NLS below $L^{2}$. International Mathematics Research Notices, 2016. 
[5] Zihua Guo, Lizhong Peng, Baoxiang Wang, and Yuzhao Wang. Uniform well-posedness and inviscid limit for the Benjamin-Ono-Burgers equation. Advances in Mathematics, 228(2):647 - 677, 2011.

[6] Martin Hadac. Well-posedness for the kadomtsev-petviashvili ii equation and generalisations. Transactions of the American Mathematical Society, 360(12):6555-6572, 2008.

[7] Martin Hadac, Sebastian Herr, and Herbert Koch. Well-posedness and scattering for the kp-ii equation in a critical space. Annales de l'Institut Henri Poincare (C) Non Linear Analysis, 26(3):917 - 941, 2009.

[8] A.D Ionescu and C.E Kenig. Local and global well-posedness of periodic KP-I equations. Mathematical Aspects of Nonlinear Dispersive Equations. Ann. Math. Stud, 163:181211, 2009.

[9] A.D. Ionescu, C.E. Kenig, and D. Tataru. Global well-posedness of the KP-I initialvalue problem in the energy space. Inventiones mathematicae, 173(2):265-304, 2008.

[10] Rafael José Iório and Wagner Vieira Leite Nunes. On equations of KP-type. Proceedings of the Royal Society of Edinburgh: Section A Mathematics, 128:725-743, 11998.

[11] Pedro Isaza and Jorge Mejía. Local and global cauchy problems for the kadomtsev-petviashvili (kp-ii) equation in sobolev spaces of negative indices. Communications in Partial Differential Equations, 26(5-6):1027-1054, 2001.

[12] B. B. Kadomtsev and V. I. Petviashvili. On the Stability of Solitary Waves in Weakly Dispersing Media. Soviet Physics Doklady, 15, December 1970.

[13] Carlos E. Kenig. On the local and global well-posedness theory for the KP-I equation. Annales de l'Institut Henri Poincare (C) Non Linear Analysis, 21(6):827 - 838, 2004.

[14] Carlos E. Kenig and Didier Pilod. Well-posedness for the fifth-order KdV equation in the energy space. Trans. Amer. Math. Soc., 367(4):2551-2612, 2015.

[15] H. Koch and N. Tzvetkov. On finite energy solutions of the KP-I equation. Mathematische Zeitschrift, 258(1):55-68, 2008.

[16] Herbert Koch and Nikolay Tzvetkov. On the local well-posedness of the Benjamin-Ono equation in $H^{s}(\mathbb{R})$. International Mathematics Research Notices, 2003(26):1449-1464, 2003.

[17] Tetsu Mizumachi and Nikolay Tzvetkov. Stability of the line soliton of the KP-II equation under periodic transverse perturbations. Mathematische Annalen, 352(3):659690, 2012.

[18] L. Molinet, J.-C. Saut, and N. Tzvetkov. Well-posedness and ill-posedness results for the Kadomtsev-Petviashvili-I equation. Duke Math. J., 115(2):353-384, 112002.

[19] L. Molinet, J.-C. Saut, and N. Tzvetkov. Global well-posedness for the KP-II equation on the background of a non-localized solution. Annales de l'Institut Henri Poincare (C) Non Linear Analysis, 28(5):653 - 676, 2011.

[20] Luc Molinet. Global well-posedness in the energy space for the Benjamin-Ono equation on the circle. Mathematische Annalen, 337(2):353-383, 2007. 
[21] Frederic Rousset and Nikolay Tzvetkov. Stability and instability of the KdV solitary wave under the KP-I flow. Communications in Mathematical Physics, 313(1):155-173, 2012.

[22] J.-C. Saut and N. Tzvetkov. On Periodic KP-I Type Equations. Communications in Mathematical Physics, 221:451-476, 2001.

[23] H. Takaoka and N. Tzvetkov. On the local regularity of the Kadomtsev-Petviashvili-II equation. International Mathematics Research Notices, 2001(2):77-114, 2001.

[24] Michael M Tom. On a generalized kadomtsev-petviashvili equation. Contemporary Mathematics, 200:193-210, 1996.

[25] Yu Zhang. Local well-posedness of KP-I initial value problem on torus in the Besov space. Communications in Partial Differential Equations, pages 1-26, 2015. 